\title{
Transport and Deposition Patterns in Drying Sessile Droplets
}

\author{
Ronald G. Larson \\ Dept. of Chemical Engineering, University of Michigan, Ann Arbor, MI 48109 \\ DOI 10.1002/aic.14338 \\ Published online February 23, 2014 in Wiley Online Library (wileyonlinelibrary.com)
}

\begin{abstract}
The literature on drying sessile droplets and deposition of suspended material is reviewed including the simple explanation of the "coffee ring" deposit given by Deegan et al. ${ }^{1}$ Analytical and numerical solutions for the flow are given, including the effect of Marangoni stresses, pinning or movement of the contact line, and viscous, thermal, gravitational, and other effects. The solution space is explored using dimensionless groups governing mass, momentum, and heat transfer effects in the droplet, external gas, and substrate. The most common types of deposition patterns are summarized, including those produced by pinned contact lines, sticking-and-slipping contact lines, and Marangoni effects. The influence of contact-line deposits is also reviewed, and the effects of colloidal, polymeric, and other depositing materials. Advanced applications from ink-jet printing to disease diagnosis are discussed as well. The review helps readers take stock of what has been learned and what remains incompletely explained. (C) 2014 American Institute of Chemical Engineers AIChE J, 60: 1538-1571, 2014
\end{abstract}

Keywords: fluid mechanics, heat transfer, deposition methods, mass transfer

\section{Introduction}

The drying of a sessile droplet of water or other volatile solvent, carrying nonvolatile solute or colloidal particles, typically leaves these materials as a nonuniform deposit or stain on the substrate. The stain pattern is highly distinctive of the heat, momentum, and mass transport processes within the droplet during evaporation, the behavior of the threephase contact line, and of the interactions of the deposited material with the interfaces of the droplet with the substrate and air. Such deposits, and their uniformity, are vital to technologies as ancient as writing with ink, and as modern as the manufacture of DNA microarrays. Intense study of the physical chemistry of drying droplets began with the publication in 1997 by Deegan et al. ${ }^{1}$ of an explanation of the ubiquitous "coffee stain" deposit formed by colloid-containing water droplets; see Figure 1. In the years since, studies of this "simple" problem have revealed that the pattern formed is influenced by a wealth of phenomena: evaporative mass transfer, heat conduction and convection, natural convection, viscous and inertial flows, surface-tension-driven flows, thermal-hydrodynamic instabilities, buoyancy effects, liquid spreading, contact-line pinning and depinning, adhesion, and others. In general, the phenomena are coupled and nonlinear, necessitating a numerical analysis to explain them. Under certain conditions, however, the drying of a sessile liquid droplet, and even the deposition pattern that results from this, can be described analytically.

This mixture of complexity and simplicity makes the drying droplet problem a fascinating one. The coupled heat, mass, and momentum transport driven by a phase change in

\footnotetext{
Correspondence concerning this article should be addressed to R. G. Larson at rlarson@umich.edu.

(C) 2014 American Institute of Chemical Engineers
}

a drying droplet raises many of the issues involved in distillation and boiling, yet the liquid confinement and controlled interfaces of a small droplet are unattainable in these other, larger-scale, flows. In addition, the drying droplet is a rich source for study of the dynamics of liquid/solid interfaces, including contact line movement. When deposition occurs from the drying droplet, issues of colloidal mechanics, surface interactions, adhesion, nucleation and crystallization arise, again in a compact and relatively controlled setting.

Because of the rapid growth of work on the drying droplet problem over the last decade and a half, it seems fitting to review what has been learned, and what remains unresolved. The first part of this review focuses on macroscopic mass, heat, and momentum transport. These transport phenomena are organized around dimensionless groups that define the dominant phenomena and the various regimes of drying, as functions of the size of the droplet, the rate of evaporation, and the properties of the fluid and substrate, and of the surrounding gas. In the latter portion of the review, I discuss the formation of the patterned deposit, including the influences of interfacial phenomena at the liquid-substrate interface and the liquid-substrate-gas contact line, including the effects of the deposited material on these phenomena. This latter discussion of interfacial phenomena is more qualitative, since theories for these are less developed, and even identification of the relevant dimensionless groups can be difficult. Experimental work in this area is not as systematic as that for the macroscopic transport. While we will find some phenomena that are common to many deposits, efforts to map out the conditions required to obtain a particular deposition pattern are in their infancy.

Perhaps the simplest case to consider is also one that most readily leads to the contact-line or "coffee-ring" stains described by Deegan et al. ${ }^{1}$ Namely, a millimeter-sized droplet of water or other volatile liquid with pinned contact line, 


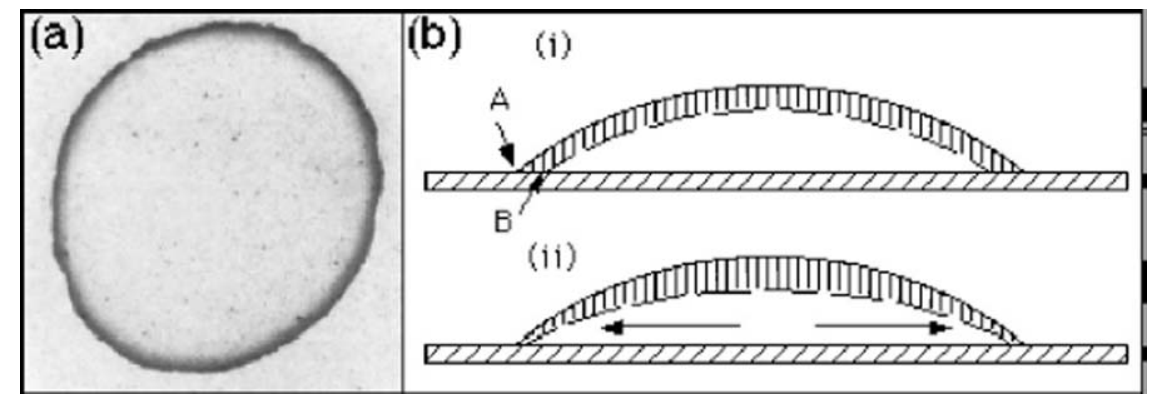

Figure 1. (a) Photograph of a dried coffee drop, whose perimeter is produced by deposited coffee particles.

The formation of the "coffee ring" results from the pinned contact line, the evaporative flux which removes liquid across the entire surface of the droplet, and the retention of the spherical-cap shape of the droplet, which drive fluid to the droplet's edge. This is illustrated in (b), where the loss of liquid due to evaporation in (1) is imagined to occur in the absence of fluid flow, which would lead to retreat of the contact line from A to $\mathrm{B}$. In reality, the pinning of the line at A forces fluid to flow to the edge to replace liquid evaporated there, as illustrated in (2). (from Deegan, ${ }^{2}$ Phys Rev E; 61:475-485, 2000. Copyright 2000 by the American Physical Society).

shaped as a spherical cap with modest contact angle, dries slowly as the liquid evaporates along its interface into stagnant gas, and is transported away from the interface by vapor-phase diffusion. In this case, the vapor-phase mass transfer and liquid phase momentum transfer reduce to simple quasi-steady linear transport problems and the vapor mass transport problem has an analytical solution that provides a boundary condition for the fluid flow problem.

If, in addition, one can also assume that variations in droplet temperature are insufficient to generate buoyancydriven or surface-tension driven flow (i.e., Marangoni flow), then the fluid flow problem has a simple analytical solution, obtained by the lubrication approximation. If one can also assume that the depositing material cannot diffuse rapidly to the substrate, but must be transported there by flow, then one can readily explain the formation of the contact-line deposit, or coffee ring. In many cases, however, the drying process leads to evaporative cooling sufficient to create a surface tension gradient that can drive Marangoni flow and/ or buoyancy-driven flow. The flow will thus be sensitive to the temperature gradient, and hence to phenomena that influence heat transfer, including the liquid and substrate thermal conductivities, the convection in the droplet, and possibly even the thermal conductivity of the gas. Still, in some cases at least, analytical solutions are possible within the lubrication approximation, even when Marangoni flow is strong.

However, the droplet might be too large to retain the shape of a spherical cap, and thus may sag or spread under gravity, and even begin to evaporate during the droplet spreading. The contact line might not remain pinned, but may move in a complex manner that depends on substrate properties and on the presence of deposited solids. The gas phase may not be stagnant; even if external air currents are blocked, the gas phase may be set in motion by buoyancy effects (i.e., natural convection) resulting from the drying of the droplet. The droplet may cool enough to reduce the vapor pressure enough to slow the overall rate of droplet drying. If the droplet contains more than one molecular component different vapor pressures of the components will likely lead to additional surface tension variations and compositional variations in the droplet and these can drive convection. If the drying is very rapid, there may be interfacial resistance to mass transfer that affects the drying rate. Rapid drying may also cause the flow in the droplet to become unstable and even turbulent, greatly affecting the mass and heat-transfer processes.
This review will not cover all these phenomena in detail, but will attempt to assess when each can be neglected or must be considered, and to describe its impact on transport. Fortunately, there is now a large body of experimental literature exploring a wide range of conditions and encompassing all of the phenomena mentioned previously. Theoretical and computational results have been less extensive so far, but scaling estimates and correlations are available in many cases, as well as some analytical and numerical results, which will also be reviewed here. The article is outlined as follows. In the section on "Typical" Values of some Droplet Parameters, I give formulas for rates of evaporation, and drying times, for sessile droplets with spherical cap shapes and tabulate data needed to use these estimates for some common liquids. In the next section, I describe the simplest case of a small sessile spherical-cap droplet, with pinned contact line, slowly evaporating in stagnant air, with no thermal effects. The flow field for this case is expressed analytically using a lubrication approximation for flat droplets. Full analytical solutions are also now available for arbitrary contact angle, for both inviscid and viscous flows, and results from these solutions will also be discussed. In the section on Dimensionless groups, I define the dimensionless groups that control the processes of momentum, heat, and mass transport. In the sections following this, the magnitudes of these groups are estimated for small for 1-mm-radius droplets composed of moderately volatile liquids, such as water, short-chain alcohols (ethanol up to hexanol) or short-chain hydrocarbons (e.g., octane). Where possible, examples are given of how the phenomena quantified by these groups affect the drying droplet transport processes. The discussion of these dimensionless groups and corresponding phenomena are grouped into sections on the effects of: droplet shape, momentum transport, thermal transport and thermally driven flows, gas-phase transport, and substrate effects. In particular, I show how Marangoni flow can be introduced into the lubrication solution and I assess thermal effects at short times after drying commences and after a quasisteady state is reached. An assessment is also given of the possible effect of thermally induced buoyancy on the droplet flow field. I also consider how buoyancy effects in the gas phase can affect the evaporation. The effect of the substrate, in particular its thickness and thermal conductivity, relative to those of the droplet, on the direction and magnitude of the Marangoni flow are considered. In the section on Moving Contact Line and Precursor Films, I consider the effect of a moving contact line on drying. In the section on Deposition Patterns, I review 
Table 1. Properties of Liquids of $20^{\circ} \mathrm{C}$ [from CRC Handbook of Chemistry and Physics, 90th ed. 2009-2010]

\begin{tabular}{|c|c|c|c|c|c|c|}
\hline & $\begin{array}{c}\text { Surface } \\
\text { tension } \mathrm{mN} / \mathrm{m}\end{array}$ & $\begin{array}{c}\text { Vapor } \\
\text { pressure kPa }\end{array}$ & $\begin{array}{l}\text { Viscosity } \\
\text { mPa.s }\end{array}$ & $\begin{array}{c}\text { Vapor diffusion } \\
\text { coefficient }^{8} \times 10^{5} \mathrm{~m}^{2} / \mathrm{s}\end{array}$ & $\begin{array}{c}\text { Evaporation latent } \\
\text { heat } \mathrm{kJ} / \mathrm{mol}\end{array}$ & $\begin{array}{c}\text { Thermal } \\
\text { conductivity } \mathrm{W} / \mathrm{m} \cdot \mathrm{K}\end{array}$ \\
\hline Ethanol & 22.27 & 5.95 & 1.17 & 1.18 & 44.32 & 0.168 \\
\hline 2-propanol & 21.7 & 4.41 & 2.43 & 1.03 & 45.39 & 0.135 \\
\hline Octane & 21.67 & 1.39 & 0.55 & 0.616 & 41.50 & 0.131 \\
\hline 1-butanol & 24.6 & 0.56 & 2.95 & 0.861 & 43.86 & 0.153 \\
\hline water & 72.58 & 2.34 & 1.002 & 2.4 & 44.23 & 0.628 \\
\hline 1-hexanol & 24.48 & 0.124 & 5.2 & 0.621 & 45.86 & 0.150 \\
\hline
\end{tabular}

the kinds of deposition patterns that can occur when salts, colloids, surfactants, and polymers are present in the liquid. Finally, I summarize and suggest major areas where more work is needed.

\section{"Typical” Values of some Droplet Parameters}

I define $\rho_{\mathrm{L}}$ to be the liquid phase density and $\mu_{\mathrm{L}}$ its viscosity. $\mathrm{U}_{\mathrm{ML}}$ is the characteristic velocity in the droplet induced by the droplet's loss of mass (i.e., in the absence of thermallydriven flows), $R$ is the droplet radius, and $\sigma$ is the liquid-air surface tension. I define $\rho_{\mathrm{vap}}(r, t)$ to be the density of the vapor phase (mass per unit volume of gas) of the evaporating liquid just above the droplet-air interface. In general, $\rho_{\text {vap }}$ will depend on the radial position $\mathrm{r}$ along the interface at time $t$, and it is usually just the saturation value at the temperature of the interface. (An exception occurs for very low-ambient pressures as discussed later.) For ambient pressures near atmospheric, the saturation vapor density is related to its vapor pressure $P_{\text {vap }}$ via the ideal gas law $P_{\text {vap }}=\rho_{\text {vap }} R_{\text {gas }} T / M$, where $R_{\text {gas }}=8.3 \mathrm{~J} /(\mathrm{mol} \mathrm{K})$ is the gas constant, $T$ the absolute temperature, and $M$ the molecular weight of the evaporating fluid, which is around $18 \mathrm{~g} / \mathrm{mol}$ for water and $46 \mathrm{~g} / \mathrm{mol}$ for ethanol. For ethanol, this gives $\rho_{\text {vap }} \approx 10^{-4} \mathrm{gm} / \mathrm{cm}^{3}$, and liquids with lower vapor pressure have proportionately lower vapor density. We take $\rho_{\mathrm{G}}$ to be the total gas density, which is approximately constant in most cases.

For droplets of water or small-molecule organic liquids, such as those tabulated in Table $1, \mu_{L} \sim 1-5 \mathrm{cP}, \rho_{L} \approx 1 \mathrm{~g} / \mathrm{cm}^{3}$ and $\rho_{\text {vap }}<10^{-4} \mathrm{~g} / \mathrm{cm}^{3}$. The mass diffusivity of vapor at room temperature and pressure is typically around $D_{\text {vap }}=0.05$ to 0.3 $\mathrm{cm}^{2} / \mathrm{s}$, while the heat diffusivity in organic liquids and water is very roughly $\alpha_{L}=k_{L} / \rho_{L} c_{p, L} \sim 10^{-3} \mathrm{~cm}^{2} / \mathrm{s}$, where $k_{L}$ is the thermal conductivity of the liquid, and $c_{p, L}$ is the liquid's heat capacity, which is around $4.19 \mathrm{~J} /(\mathrm{gK})$ for water at room temperature and around half of this for ethanol. The surface tension $\sigma$ of the liquid is around 20-25 dyn/cm for organic liquids and around $70 \mathrm{dyn} / \mathrm{cm}$ for clean water. Droplets of radius $R=1$ $\mathrm{mm}$, composed of liquids with saturation vapor pressures either less than, or at least not much higher than, that of water, i.e., below around $5 \mathrm{kPa}$, dry out at room temperature and pressure at rates of $-\dot{m} \approx 5 \times 10^{-6} \mathrm{gm} / \mathrm{s}$ or less, as can be inferred from the average rate of evaporative mass transfer given by ${ }^{3}$

$$
\begin{aligned}
-\dot{m} & \approx \pi R D_{\text {vap }}(1-H) \rho_{\text {vap }}\left(0.27 \theta^{2}+1.30\right) \\
& \approx 5 R D_{\text {vap }}(1-H) \rho_{\text {vap }}(\text { for } \theta=\pi / 6)
\end{aligned}
$$

where $H$ is the relative vapor pressure (or humidity for water) far from the droplet and can be taken to be zero if the vapor is absent from the ambient air far from the droplet. Here $\rho_{\text {sat }}$ is the saturation concentration of vapor at the temperature of the droplet surface. The first expression in Eq. 1 is a reason- ably accurate (within a few percent) for contact angles $\theta$ between 0 and $90^{\circ}$. The expression with the coefficient " 5 " on the right side of the above is obtained for a contact angle of $30^{\circ}$ or so. For very small contact angles, this prefactor should be 4 , while for a $45^{\circ}$ angle, it should be close to 6 . Here we are assuming that the droplet temperature is close enough to the ambient temperature of the surrounding gas that the saturation vapor pressure is the value at ambient temperature. The relationship between droplet volume and time given by Eq. 1 agrees with experimental measurements of Birdi et $\mathrm{al}^{4,5}$ and Rowan et al. ${ }^{6,7}$ for droplets with pinned contact line, and yields a nearly linear relationship between droplet volume and time for contact angles less than $40^{\circ}$.

Droplets of modest height to radius ratio $h_{0} / R$ have volumes of very roughly $V \approx h_{0} R^{2}$ and for $H=0$ completely evaporate in a drying time of around

$$
t_{f} \approx \rho_{L} V /(-\dot{m}) \approx 0.2 \frac{\rho_{L}}{\rho_{\text {vap }}} \frac{R h_{0}}{D_{\text {vap }}}
$$

For the "typical droplet" considered here, with $h_{0} / R$ of around 0.5 , this gives a drying time $t_{f}$ ranging from around $200 \mathrm{~s}$ for the most volatile liquid in Table 1 (ethanol), up to $10,000 \mathrm{~s}$ (i.e., around $3 \mathrm{~h}$ ) for the least volatile (hexanol).

If the flow in the droplet is entirely due to the loss of mass, its characteristic velocity near the start of the drying process will be roughly $\mathrm{U}_{\mathrm{ML}} \sim R / t_{f} \sim 5 \times 10^{-4} \mathrm{~cm} / \mathrm{s}=5$ $\mu \mathrm{m} / \mathrm{s}$ for a drying time of $t_{f}=200 \mathrm{~s}$ and as low as $\mathrm{U}_{\mathrm{ML}}$ $\sim 10^{-5} \mathrm{~cm} / \mathrm{s}=0.1 \mu \mathrm{m} / \mathrm{s}$ for the less volatile liquids in Table 1. As drying progresses, this velocity increases and can be estimated at any point in the drying by taking $t_{f}$ to be the time remaining before drying is complete. I will discuss thermocapillary effects shortly, which can greatly increase the characteristic velocity $\mathrm{U}$, well above the mass-loss value $\mathrm{U}_{\mathrm{ML}}$. A formula for the height-averaged velocity will be given below, which supports this scaling law for $\mathrm{U}_{\mathrm{ML}}$.

From Eq. 1, the average evaporative mass loss per unit area of substrate surface (i.e., the mass flux) is given by

$$
\begin{aligned}
J_{\mathrm{ave}} & \equiv \frac{-\dot{m}}{\pi R^{2}} \approx \frac{D_{\mathrm{vap}}(1-H) \rho_{\mathrm{vap}}}{R}\left(0.27 \theta^{2}+1.30\right) \\
& \approx 1.6 \frac{D_{\mathrm{vap}} \rho_{\mathrm{vap}}}{R}(\text { for } \theta=\pi / 6, H=0)
\end{aligned}
$$

which is around $10^{-4} \mathrm{~g} / \mathrm{cm}^{2} \mathrm{~s}$ or less, for vapor concentrations of $\rho_{\text {vap }}<10^{-4} \mathrm{~g} / \mathrm{cm}^{3}$. The average rate of heat loss due to latent heat of evaporation is then $J_{\text {ave }} \Delta H_{\text {vap }}$, where $\Delta H_{\text {vap }}$ is the heat of vaporization per unit mass of liquid. If this is balanced by steady-state heat conduction from the substrate, whose rate is around $k_{L} \Delta T / h_{0}$, where $k_{L}$ is the thermal conductivity of the liquid, one can estimate the steady-state temperature change from the bottom to the top of the droplet due to drying as 


$$
\begin{gathered}
\Delta T \approx \frac{J_{\text {ave }} \Delta H_{\text {vap }} h_{0}}{k_{L}} \approx \frac{\Delta H_{\text {vap }} h_{0} D_{\text {vap }}(1-H) \rho_{\text {vap }}}{k_{L} R}\left(0.27 \theta^{2}+1.30\right) \\
\approx \frac{2 \Delta H_{\text {vap }} D_{\text {vap }} \rho_{\text {vap }}}{k_{L}} \frac{h_{0}}{R}(\text { for } \theta<\pi / 2, H=0)
\end{gathered}
$$

In the last approximation in Eq. 4, the contact angle is taken to be less $90^{\circ}$, and the prefactor " 2 " is a rough approximation over this range, only valid to within $30 \%$ or so, but accurate enough for a simple estimate. For a molecular weight of around $50 \mathrm{~g} / \mathrm{mol}$ (close to that of ethanol), according to Table $1, \Delta H_{\text {vap }}$ is around $10^{3} \mathrm{~J} / \mathrm{gm}$. (Note that the values for latent heat given in Table 1 are per mole, rather than per unit mass.) For $\theta=\pi / 4$, corresponding to $h_{0} / R=0.5$, this yields a temperature drop of around $5^{\circ} \mathrm{C}$ for the more volatile liquids in Table 1 down to $0.1^{\circ} \mathrm{C}$ for the less volatile ones.

Based on the aforementioned estimates, the values for the dimensionless groups for the "typical" droplet are estimated shortly, along with the implications for transport. The aforementioned estimates of evaporation rates, fluxes and times in Eqs. 1-3 can readily be rescaled for smaller droplets using the earlier formulas. A picoliter droplet of ethanol, with radius $R \approx 10 \mu \mathrm{m}$, for example, would be expected to dry out in $\sim 0.02 \mathrm{~s}$. Thus, by using such scaling rules, the behavior of droplets too small or too rapidly drying to be easily observed, may be inferred. One must be careful, however, to check that assumptions valid for the larger droplets still apply for the smaller ones; dimensionless groups given below can help in this determination. With the same caveat, the aforementioned formulas can also be applied to droplets much larger than $R=1 \mathrm{~mm}$, as long as one notes that they will sag under gravity, and, thus, have heights that are roughly independent of radius and of contact angle. If the contact line is not pinned, but retreats during drying, the scaling formulas are similar but with different prefactors.

\section{Simplest Case: Spherical-Cap shape, Pinned Contact Line, Quasi-Steady Diffusive Transport}

The analysis of mass transport from a drying droplet, and the resulting fluid flow within the droplet is greatly simplified when the evaporating droplet retains the shape of a spherical cap, with pinned contact line. The spherical cap shape is represented by

$$
h(r, t)=\sqrt{R^{2} / \sin ^{2} \theta(t)-r^{2}}-R / \tan \theta(t)
$$

where $h(r, t)$ is the local height of the droplet, which is a function of radial coordinate $r$, and is a function of time $t$ through the time dependence on contact angle $\theta(t)$. (The contact angle must change as the droplet dries if the contact line is fixed and droplet radius $R$ is, thus, constant. If the droplet radius shrinks, and the spherical-cap shape is maintained, then $R(t)$ is a function of time.) To a good approximation, Eq. 5 can be simplified for relatively flat droplets to a parabolic profile

$$
h=h(0, t)\left(1-\tilde{r}^{2}\right)
$$

where $\tilde{r} \equiv r / R$ is the dimensionless droplet radius. The contact angle in this case is given by $\theta(t)=2 h(0, \mathrm{t}) / \mathrm{R}$. The initial height of the droplet is denoted by $h_{0}=h(0,0)$. The droplet will retain this shape if it is too small to sag under gravity, and the flow in the droplet is too feeble to distort the interface shape.
Additional simplifications that sometimes hold include that the droplet evaporates slowly enough into stagnant air that mass transfer is vapor-phase diffusion limited, the flow within the droplet is of low Reynolds number, and the heat, mass, and momentum transport processes are all at quasi-steady state. The conditions under which these simplifications are reasonable are discussed later using dimensionless groups that we define in the next section. We will also estimate values for these dimensionless groups for "typical conditions" that we define in the sections following this. In the simplest case, the problem reduces to solving only the quasi-steady-state vapor concentration field, which is controlled by vapor diffusion, and the quasi-steady-state droplet velocity field, which is controlled by the evaporative mass loss and viscous drag. The geometry is that of a spherical cap. These two problems reduce to a Laplace equation for vapor field above the droplet, and a Stokes flow problem within the droplet. The coupling between these problems is one-way: the vapor concentration field determines the flux of vapor from the droplet interface, and this, combined with a mass balance and the requirement that when the droplet shrinks it retains a spherical-cap shape with a pinned contact line, establishes a height-averaged radial liquid velocity that must be attained to enforce these conditions. This problem was first solved in a simplified manner by Deegan et al., ${ }^{1}$ in a now-famous article that first explained the "coffee ring" effect.

The vapor concentration field can be derived from a solution of Laplace's equation with boundary conditions that (1) the vapor density is fixed at $\rho_{\text {vap }}$ along the spherical-cap surface, (2) the vapor flux is zero along the substrate surface not covered by liquid, and (3) the vapor concentration is constant at a value of $H \rho_{\text {vap }}$ far above the droplet surface. Here $H$ is the fractional "humidity" or the equivalent of humidity for nonaqueous droplets. The analytical solution to the Laplace equation in this geometry was given by Lebe$\operatorname{dev}^{9}$ and Picknett and Bexon. ${ }^{10}$ For relatively flat droplets, the flux of vapor from the surface of the droplet that is derived from this solution can be approximated by

$$
J(r, \theta)=J_{o}(\theta)\left(1-\tilde{r}^{2}\right)^{-\lambda}
$$

where the exponent $\lambda$ is a function of the contact angle $\lambda(\theta)$ $=0.5-\theta / \pi$ with $\theta$ in radians, and $J_{o}(\theta)$ given by

$$
J_{o}(\theta)=\frac{\rho_{\mathrm{vap}}(1-H)}{R}\left(\frac{(8 \pi-4)(\theta-\pi / 2)^{2}}{\pi^{3}}+1\right)
$$

( $\mathrm{Hu}$ and Larson $^{3}$ suggested the aforementioned equation as a modification to the original formula of Deegan et al. $\lambda(\theta)=(\pi-2 \theta) /(2 \pi-2 \theta)$. This result of Deegan et al. ${ }^{1}$ applies when the flux is fit to the equation $J(r) \propto(1-\tilde{r})^{-\lambda}$ rather than to the form in Eq. $7, J(r) \propto\left(1-\tilde{r}^{2}\right)^{-\lambda}$, which was introduced by $\mathrm{Hu}$ and Larson. ${ }^{3}$ The form used by Deegan et al. ${ }^{1}$ is asymptotically correct near the contact line, but seems to be less accurate overall than the form introduced by $\mathrm{Hu}$ and Larson. Note that either Eq. 7 or the formula of Deegan et al. gives an evaporative flux that is singular at the contact line, when the contact angle $\theta$ is less than $\pi / 2$ or $90^{\circ}$, so that $\lambda$ in Eq. 7 is greater than zero. Eq. 7 agrees quite well with a finite element solution for the evaporation flux by Widjaja and Harris ${ }^{11}$

Integrating the evaporation flux in Eqs. 7 and 8 over the droplet surface yields the overall rate of mass loss from the droplet, given earlier in Eq. 1. 
From the vapor flux boundary condition, and a mass balance, the height-averaged velocity can be derived

$$
\tilde{\bar{u}}_{r} \equiv \frac{\tilde{u}_{r} t_{f}}{R}=\frac{1}{4} \frac{1}{1-\tilde{t}} \frac{1}{\tilde{r}}\left[\left(1-\tilde{r}^{2}\right)^{-\lambda(\theta)}-\left(1-\tilde{r}^{2}\right)\right]
$$

where $\tilde{t} \equiv \frac{t}{t_{f}}$ and $\tilde{r} \equiv \frac{r}{R}$

This height-average velocity has a singularity at the contact line, where $\tilde{r} \rightarrow 1$, which arises from the singularity in evaporation rate at the contact line. To remove this singularity, Fischer ${ }^{13}$ and Masoud and Felske ${ }^{14}$ took the evaporation rate to fall rapidly (exponentially) toward zero very near the contact line. Another way to remove the singularity in velocity is to relax the no-slip boundary condition so that a finite velocity occurs near the contact line. Either method allows formulas for the full velocity field for droplets of arbitrary contact angle to be derived analytically for spherical-cap droplets using toroidal coordinates, or for cylindrical-cap droplets using biharmonic coordinates, for either pinned or moving contact lines. ${ }^{14,15}$ Such solutions are complex however, and to show the main features, we will first invoke the lubrication approximation to obtain the velocity field from the height-averaged radial velocity of Eq. 9. In this approximation, valid for relatively flat droplets, one simply assumes a parabolic velocity profile over the local height $h(r, t)$ of the droplet, with a no-slip condition on the substrate $(z=0)$ and a no-shear-stress condition at the interface between the droplet and air $(z=h(r, t))$. One also requires that the integral of this velocity profile, divided by $h(r, t)$, must yield the heightaveraged profile given in Eq. 9. The lubrication approximation assumes that the flow field is predominantly radial, with the largest gradient in radial velocity being the gradient in the axial direction; i.e. $\partial v_{r} / \partial z \gg \partial v_{r} / \partial r$. This should be approximately true for rather flat droplets. Invoking this approximation yields

$$
\begin{gathered}
\tilde{u}_{r}=\frac{3}{8} \frac{1}{1-\tilde{t}} \frac{1}{\tilde{r}}\left[\left(1-\tilde{r}^{2}\right)-\left(1-\tilde{r}^{2}\right)^{-\lambda(\theta)}\right]\left(\frac{\tilde{z}^{2}}{\tilde{h}^{2}}-2 \frac{\tilde{z}}{\tilde{h}}\right) \\
+\frac{\tilde{r} h_{o}^{2} \tilde{h}}{R^{2}}\left(\tilde{J} \lambda(\theta)\left(1-\tilde{r}^{2}\right)^{-\lambda(\theta)-1}+1\right)\left(\frac{\tilde{z}}{\tilde{h}}-\frac{3}{2} \frac{\tilde{z}^{2}}{\tilde{h}^{2}}\right)
\end{gathered}
$$

where the dimensionless variables are $\tilde{u}_{r} \equiv \frac{u_{r} t_{f}}{R} ; \quad \tilde{u}_{z} \equiv \frac{u_{z} t_{f}}{h_{o}}$; $\tilde{t} \equiv \frac{t}{t_{f}} ; \tilde{r} \equiv \frac{r}{R} ; \tilde{z} \equiv \frac{z}{h_{o}} ; \tilde{h} \equiv \frac{h}{h_{o}}$ and $\tilde{J} \equiv \frac{J(0, \theta)}{\dot{h}}$. As before, $t_{f}$ is the drying time, $R$ is the contact line radius, and $h_{o}$ is the initial height of the droplet. Also $\dot{h} \equiv-\partial h(0, t) / \partial t$, which appears in the denominator of the definition of $\tilde{J}$, is the time rate of change of droplet height. The normal lubrication approximation, which assumes that the shear stress at the surface of the droplet is just $\mu_{L} \partial v_{r} / \partial z$, yields only the first term in the aforementioned expression. However, in principle, the shear stress is the sum of $\mu_{L} \partial v_{r} / \partial z$ plus $\mu_{L} \partial v_{z} / \partial r$, the latter term normally taken to be small both because the axial velocity is smaller than the radial velocity and because the radial length scale $R$ is larger than the vertical scale $h_{o}$. However, both velocities become singular at the contact line, and derivatives with respect to radial coordinate $\mathrm{r}$ become even more singular, due to the singularity at $r=R$ in Eq. 9. Since the singularity becomes stronger as the droplet gets flatter, the neglect of the higher-order term no longer becomes rigorously valid as the droplet becomes arbitrarily flat. Thus, it turns out that retention of the "higher order" term $\mu_{L} \partial v_{z} / \partial r$, yields an improved approximation to the velocity field, as revealed by finite element analysis of the full Stokes equation without the lubrication approximation. ${ }^{12}$ The contribution of the added higher order term is underlined in Eq. 10.

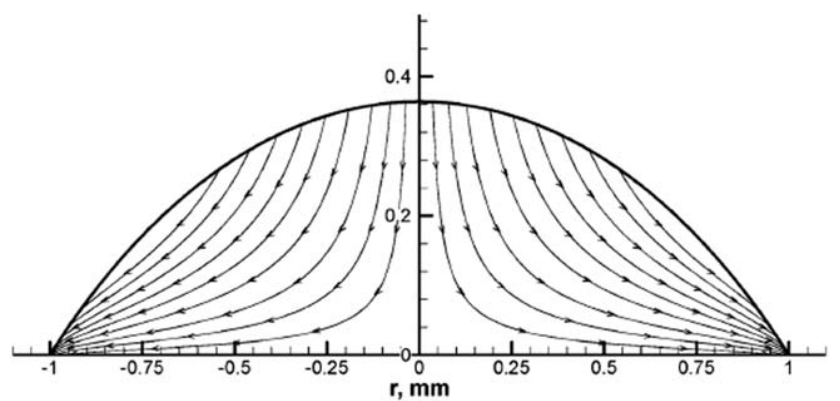

Figure 2. Velocity field calculated by the finite element method for a droplet with a pinned contact line radius of $1.0 \mathrm{~mm}$, drying time of $360 \mathrm{~s}$.

(Reprinted with permission from $\mathrm{Hu}$ and Larson, ${ }^{12}$ Langmuir; 21:3972-3980, 2005. Copyright 2005 American Chemical Society).

Using the known radial velocity $\tilde{u}_{r}$ in Eq. 10, the vertical velocity $\tilde{u}_{z}$ can be derived through the continuity equation, giving

$$
\begin{gathered}
\tilde{u}_{z}=\frac{3}{4} \frac{1}{1-\tilde{t}}\left[1+\lambda(\theta)\left(1-\tilde{r}^{2}\right)^{-\lambda(\theta)-1}\right]\left(\frac{\tilde{z}^{3}}{3 \tilde{h}^{2}}-\frac{\tilde{z}^{2}}{\tilde{h}}\right) \\
+\frac{3}{2} \frac{1}{1-\tilde{t}}\left[\left(1-\tilde{r}^{2}\right)-\left(1-\tilde{r}^{2}\right)^{-\lambda(\theta)}\right]\left(\frac{\tilde{z}^{2}}{2 \tilde{h}^{2}}-\frac{\tilde{z}^{3}}{3 \tilde{h}^{3}}\right) \tilde{h}(0, \tilde{t}) \\
-\frac{h_{o}^{2}}{R^{2}}\left(\tilde{J} \lambda(\theta)\left(1-\tilde{r}^{2}\right)^{-\lambda(\theta)-1}+1\right)\left(\tilde{z}^{2}-\frac{\tilde{z}^{3}}{\tilde{h}}\right) \\
-\frac{\tilde{r}^{2} h_{o}^{2}}{R^{2}} \tilde{J} \lambda(\theta)(\lambda(\theta)+1)\left(1-\tilde{r}^{2}\right)^{-\lambda(\theta)-2}\left(\tilde{z}^{2}-\frac{\tilde{z}^{3}}{\tilde{h}}\right) \\
+\frac{\tilde{r}^{2} h_{o}^{2}}{R^{2}}\left(\tilde{J} \lambda(\theta)\left(1-\tilde{r}^{2}\right)^{-\lambda(\theta)-1}+1\right)\left(\frac{\tilde{z}^{3}}{\tilde{h}^{2}}\right) \tilde{h}(0, \tilde{t})
\end{gathered}
$$

Again, the underlined terms are the result of inclusion of the higher-order term in the radial velocity.

The flow field obtained from this lubrication approximation is surprisingly accurate, even for contact angles as large as $40^{\circ}$, as shown by comparison to finite element solutions. ${ }^{12}$ An illustrative velocity field from a finite-element solution is shown in Figure 2. A similar flow field was obtained analytically without the lubrication approximation, and with the contact line singularity removed, by Masoud and Felske. ${ }^{14}$ The effect of the evaporation flux profile was explored by Petsi and Burganos ${ }^{15}$ for a cylindrical-cap droplet in Figure 3. Note in Figure 3 that for a contact angle of $60^{\circ}$ the flows are similar for uniform flux and diffusion controlled flux, even though the latter becomes singular (or nearly so) at the contact line. The effect of the singularity at the contact line is weaker, however, for a contact angle of $60^{\circ}$ than for smaller angles, and in fact diffusion-controlled evaporative flux is uniform in when $\theta$ reaches $90^{\circ}$ (see Eq. 7). Also, the effect of the singularity should be weaker for a cylindrical cap than for a spherical one.

Figure $4 \mathrm{a}$, which is the left side of Figure 4, shows the streamlines in a spherical-cap droplet for the case of a contact angle of $90^{\circ}$, for which the evaporative flux is uniform along the droplet surface. Shown in Figure $4 b$, which is the right half of Figure 4, is the solution for an inviscid fluid, subject to the same boundary conditions except that the no-slip condition on the substrate is dropped. The inviscid flow solution 

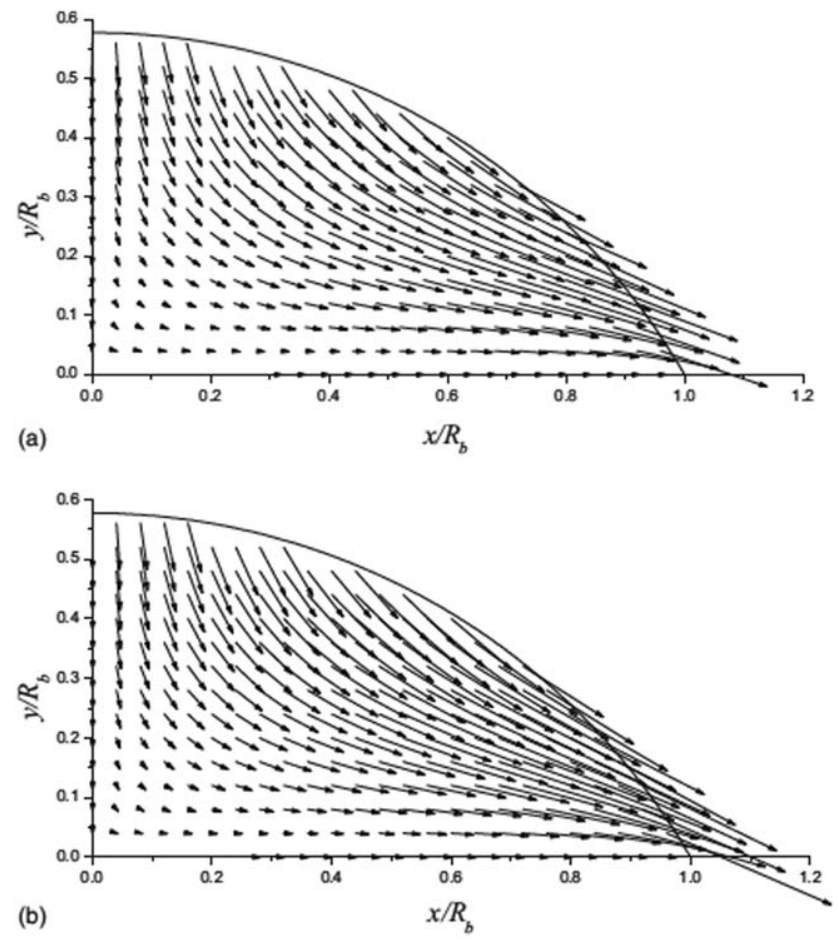

Figure 3. The flow field inside an evaporating cylindrical-cap droplet with pinned contact line, contact angle $\theta=\pi / 3=60^{\circ}$ and slip length divided by droplet radius of $10^{-3}$.

(a) uniform evaporation rate, and (b) diffusioncontrolled evaporation rate (from Petsi and Burganos, ${ }^{15}$ Phys Rev E; 78:036324, 2008. Copyright 2008 by the American Physical Society).

was derived by Tarasevich ${ }^{16}$ as an infinite sum of Legendre polynomials. For tall droplets (e.g., those with $90^{\circ}$ contact angle), there is a remarkable similarity between the viscous and the inviscid solutions, because the influence of the no-slip boundary condition is negligible on the flow field near the top of the droplet, which is dominated by the mass outflow due to evaporation. For a droplet with a contact angle of $40^{\circ}$, the viscous flow differs significantly from the inviscid flow, as can be seen in a direct comparison of the two in Masoud and Felske. ${ }^{14}$ Nevertheless, the qualitative features of the flow remain the same: there is a downward and outward flow, induced by the constraints of the pinned contact line and the spherical-cap shape of the droplet. These constraints mean that fluid that evaporates from the droplet edge must be largely replaced by fluid flowing from the center of the droplet. The finer details of the flow depend on the evaporative flux profile, the droplet height, and the no-slip condition. For viscous flow, removal of the contact line singularity using a slip coefficient has little effect on the flow pattern, as long the slip length is much smaller (1000-fold smaller) than the droplet radius. ${ }^{15}$ (The "slip length" is the product of slip velocity and fluid viscosity, divided by shear stress.)

Apart from the contact line singularity in velocity, the flow depicted in Figure 2 is weak, of order a few microns/s. The case considered in Figure 2 is typical of $1 \mathrm{~mm}$ radius water droplets, or droplets of similar volatility, at room conditions. As we shall see in the next section, we normally expect that even small temperature variations along the surface of the droplet, produced by latent heat of evaporation, will lead to velocities much larger than this, and so the solu- tion shown in Figures 2-4 should rarely be valid. If one slows the rate of evaporation greatly to reduce temperature gradients, this will also slow the base flow shown in Figure 2 to the same degree, since the velocity field is linear in the evaporation rate. Thus, as long as the flow field is linear in the evaporation rate, the relative importance of Marangoni flow is insensitive to the droplet volatility. Nevertheless, surprisingly, for water droplets, the Marangoni flow is often very weak, much weaker than predicted. This has not been fully explained, but is thought to arise from the ease with which water droplet surfaces become contaminated by surface-active agents leached from solid surfaces or absorbed from the air. Were Marangoni flow as strong in water as it theoretically should be for pure water, it is doubtful that the "coffee ring" phenomenon would be a common observance.

The simple flow fields discussed in in this section are only valid when multiple complicating effects can be neglected, the strengths of which are governed by various dimensionless groups defined in the next section. There are a large number of such groups, which I will consider one at a time in the four sections subsequent to this. In these sections, I will estimate the values of these dimensionless groups for "typical" droplets of moderately volatile liquids of around 1 $\mathrm{mm}$ radius, at atmospheric pressure and temperature, and assess the implications for droplet transport. I will first consider the dimensionless groups governing droplet shape, then those governing viscous flow, then ones governing thermal effects, substrate properties, and gas-phase properties.

\section{Dimensionless Groups}

Eight continuum quantities controlling droplet shape and fluid flow in the droplet are $t_{f}, h_{0}, R, \rho_{L}, U, v_{L}, \sigma$, and $g$, which are the droplet drying time, droplet height, radius, liquid density, characteristic velocity, kinematic viscosity, the droplet/air surface tension, and the gravitational constant, respectively. From these eight quantities, five dimensionless groups can be formed. The quantities $t_{f}, h_{0}$, and $\rho_{L}$ can be used to define basic units of time, distance, and density (or mass), from which dimensionless groups are formed for each of the remaining five quantities. Following this procedure, we obtain first the geometric aspect ratio $h_{0} / R$ of droplet

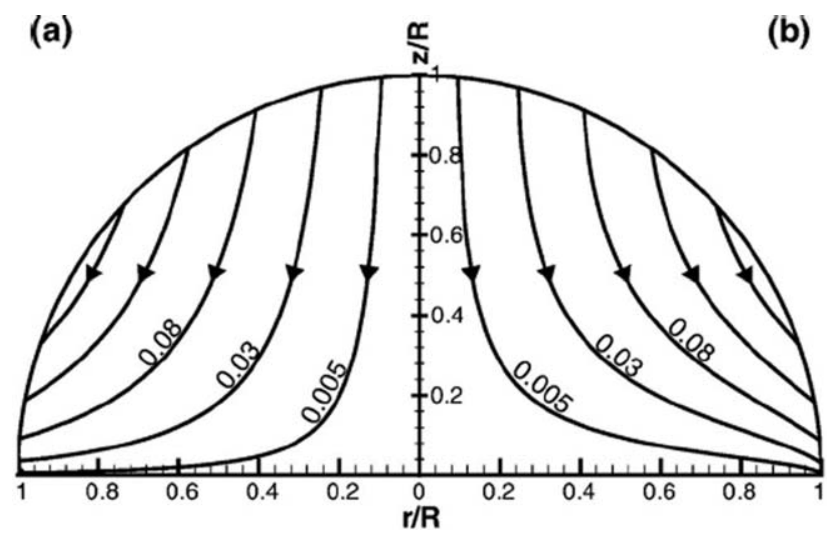

Figure 4. Streamlines for spherical-cap droplet with contact angle $\theta=90^{\circ}$ (and therefore uniform diffusive evaporative flux) and pinned contact line for (a) inviscid, and (b) viscous flow (Reprinted with permission from Masoud and Felske, ${ }^{14}$ Phys Fluids; 21:042102, 2009. Copyright 2009, AIP Publishing LLC). 
height to radius. Next is the Strouhal number $\mathrm{Sr} \equiv U t_{f} / R$ defining the ratio of drying time to the time needed for a fluid particle to traverse the droplet radius by flow. As discussed earlier, if the flow in the droplet is entirely due to the loss of mass, its characteristic velocity is roughly $U=U_{M L}$ $\sim R / t_{f}$, and so in this case the Strouhal number is unity, a trivial result. However, thermal effects, such as Marangoni flows can produce much higher velocities $U$, and so the Strouhal number will then be very large, and it will enter our discussion of thermal effects shortly. Next, is the Reynolds number $R e \equiv U h_{0} / v_{L}$, which governs the ratio of convection to diffusion of momentum. Then, the capillary number $\mathrm{Ca} \equiv \mu_{L} U / \sigma$ is the ratio of viscous to surface tension forces (note that $\mu_{L}=v_{L} \rho_{L}$ ), and finally the Bond number Bo $\equiv \rho_{L} g h_{0} R / \sigma$ is the ratio of gravitational to surface tension forces. I note in passing that the combination (Bo/ $\mathrm{Ca})\left(h_{0} / R\right)=g h_{0}^{2} / v_{L} U \equiv \mathrm{St}$ is the Stokes number, ${ }^{18}$ sometimes invoked to describe the ratio of gravitational to viscous forces. Since it is a combination of other dimensionless groups that are more useful for our purposes, I consider it no further. I also note that of the eight quantities above, two of them, the drying time $t_{f}$ and the characteristic fluid velocity $U$, are dependent variables that are controlled by the mass and momentum transport physics. For example, in the simplest case discussed earlier, the drying time $t_{f}$ is given by Eq. 2 in terms of the vapor diffusivity, droplet dimensions, and liquid and vapor densities, and the scale of the fluid velocity set by the droplet rate of mass loss is simply $U_{M L} \sim R / t_{f}$

Next, we introduce seven independent quantities governing thermal effects in the droplet, namely $k_{L}$, which is the liquid thermal conductivity, $c_{p, L}$, the liquid heat capacity, $\Delta H_{\mathrm{vap}}$, the heat of vaporization, $\Delta T$, the temperature difference across the droplet $\beta \equiv-d\left(\ln \rho_{L}\right) / d T$, the thermal expansion coefficient $d \sigma / d T$, the temperature coefficient of surface tension, and $\beta_{\text {vap }} \equiv d\left(\ln \rho_{\text {vap }}\right) / d T$, the temperature coefficient of equilibrium vapor density. We note that $\alpha_{L}$, the thermal diffusivity, is obtained from the thermal conductivity $k_{L}$, heat capacity, and density by $\alpha_{L} \equiv k_{L} /\left(c_{p, L} \rho_{L}\right)$. To avoid adding heat and temperature as new dimensional units, we group these quantities so that units of heat and temperature disappear. We obtain the following five quantities: $\alpha_{L}, \Delta H_{\mathrm{vap}} /\left(c_{p, L} \Delta T\right)$, $\beta \Delta T,(d \sigma / d T)) \Delta T$ and $\beta_{\text {vap }} \Delta T$. Using parameters from our earlier list, we can turn these quantities into five dimensionless groups. First $\alpha_{L}$ is made dimensionless in the Prandtl number $\operatorname{Pr} \equiv v_{L} / \alpha_{L}$, which is the ratio of liquid momentum to thermal diffusivities. An alternative to the Prandtl number $\operatorname{Pr}$ is the product group $\operatorname{PrRe}=U h_{0} / \alpha_{L}$, which is the ratio of convective to diffusive heat transport and, is, therefore, the heat transfer counterpart of the Reynolds number Re. (PrRe can also be thought of as an inverse Stanton number, the ratio of heat convection to heat diffusion.) Next, we make the ratio of heat capacity to latent heat dimensionless through the evaporation number $E \equiv\left(\frac{\alpha_{L}}{U h_{0}}\right)\left(\frac{c_{p, L} \Delta T}{\Delta H_{\text {vap }}}\right)=\frac{1}{\operatorname{RePr}}\left(\frac{c_{p, L} \Delta T}{\Delta H_{\text {vap }}}\right)$. If a temperature difference is imposed (for example by making the substrate temperature differ from the air temperature), then $E$ is an independent control variable, but if not, $E$ can be considered to be a dependent variable, determined by solution of the heat equation. Next, we make the thermal expansion coefficient dimensionless using the buoyancy number $\mathrm{B}_{\mathrm{U}} \equiv \mathrm{g} \beta \Delta \mathrm{Th}_{\mathrm{o}}{ }^{2} \mathrm{t}_{\mathrm{f}} / v_{\mathrm{L}} \mathrm{R}$, which is a ratio of buoyancy-driven convection to the mass-loss velocity $R / t_{f}$. A more commonly used alternative to this is the liquid phase Grashof number
$G r_{L} \equiv \beta \Delta T g R^{3} / v_{L}^{2}$, but the buoyancy number will be more useful for our purposes. Then, the temperature coefficient of surface tension is made dimensionless in the same way as the buoyancy number to form the Marangoni number $M a_{U} \equiv-\frac{d \sigma}{d T} \frac{\Delta T t_{f} h_{0}}{\mu_{L} R^{2}}$. Again, this definition, while different from other choices, serves our purposes in what follows. Finally, the temperature coefficient of equilibrium vapor pressure was already made dimensionless above by having multiplied it by the temperature difference $\beta_{\text {vap }} \Delta T$. These definitions will be discussed in more detail following.

In the gas phase, the properties that matter are the vapor diffusion coefficient $D_{\text {vap }}$ and vapor density $\rho_{\text {vap }}$, as well as the gas-phase density $\rho_{G}$, kinematic viscosity $v_{G}$, and thermal conductivity $k_{G}$. From these, we can form five dimensionless groups, namely the dimensionless vapor diffusivity $D_{\text {vap }} t_{f} / R h_{0}$, the vapor density relative to liquid density $\rho_{\text {vap }} / \rho_{L}$, the gas-to-liquid density ratio $\rho_{G} / \rho_{L}$, the gas-phase Grashof number $G r_{G} \equiv\left(\frac{\rho_{\text {vap }}}{\rho_{G}}\right)\left(\frac{g R^{3}}{v_{G}{ }^{2}}\right)$, and the ratio of thermal conductivities of gas to liquid $k_{G} / k_{L}$. (The latter is around 0.04 for a water droplet in air, and around 0.15 for organic liquids in air.)

Finally, the thermal properties of the substrate are its thermal conductivity $k_{S}$, thermal diffusivity $\alpha_{S} \equiv k_{S} / c_{p, S} \rho_{S}$, and thickness $h_{S}$, from which we can form three dimensionless groups, namely $k_{L} / k_{S}$, the ratio of liquid to substrate thermal conductivities $k_{L} h_{S} / k_{S} / h_{0}$, the ratio of thermal resistivity of the substrate to that of the liquid, and $h_{S}{ }^{2} / \alpha_{S} t_{f}$, which is the ratio of substrate thermal equilibration time to the droplet drying time. This assumes that the substrate has large lateral dimensions compared to the droplet radius. The wetting properties of the substrate are also relevant, but the most important of these, the initial contact angle $\theta_{0}$, is already implicitly included in our list of dimensionless groups, through the droplet aspect ratio $h_{0} / R$, which for relatively flat droplets is related to the contact angle by $\theta_{0}=2 h_{0} / R$.

We noted earlier that the drying time $t_{f}$ is really a dependent variable that depends on the vapor phase diffusivity. For example, for the simple drying case, we have $t_{f} \approx 0.2 \frac{\rho_{L}}{\rho_{\text {vap }}} \frac{R h_{0}}{D_{\text {vap }}}$, giving for the dimensionless group $D_{\text {vap }} t_{f} f$ $R h_{0}$ the value of $0.2 \rho_{L} / \rho_{\text {vap. }}$. Because of this dependency, the dimensionless group $D_{\text {vap }} t_{f} / R h_{0}$ is really a dependent variable, namely a dimensionless drying time scaled by vapor diffusivity and obtainable from other dimensionless groups (e.g., $\rho_{\text {vap }} / \rho_{L}$ ) through solution of the mass transport problem. Another dependent variable is the velocity $U$, and we can consider the Strouhal number $\mathrm{Sr}=U t_{f} / R$ to be a dimensionless velocity obtained from the Navier-Stokes (or momentum-balance) equations. Finally, if no temperature difference is imposed across the droplet, a dimensionless temperature difference, for example either $E \equiv \frac{1}{\operatorname{RePr}}\left(\frac{c_{p} \Delta T}{\Delta H_{\text {vap }}}\right)$, or alternatively, simply, $\left(\frac{c_{p} \Delta T}{\Delta H_{\text {vap }}}\right)$ can also be taken as dependent variable obtained from the heat equation. We can show this explicitly using Eq. 4, which is the simplified solution of the heat equation for the temperature drop $\Delta T$, written in terms of the ratio $\Delta H_{\text {vap }} D_{\text {vap }} \rho_{\text {vap }} / k_{L}$. The dimensionless temperature that is formed by dividing $\Delta T$ by this ratio can be expressed in terms of our other dimensionless groups as $\frac{k_{L} \Delta T}{\Delta H_{\text {vap }} D_{\text {vap }} \rho_{\text {vap }}}=E \cdot \operatorname{Sr}\left(\frac{\rho_{L}}{\rho_{\text {vap }}}\right) /\left(\frac{D_{\text {vap }} t_{f}}{R h_{0}}\right)$. Thus, Eq. 4 can be rewritten in terms of our dimensionless groups as 
Table 2. Eighteen Dimensionless Groups Controlling Droplet Drying and Typical Values for Small Droplets (Radius 1 mm) of Moderately Volatile Liquids Evaporating in Air at Ambient Conditions with no External Heating on Substrates of Thickness 0.1 to $10 \mathrm{~mm}$

\begin{tabular}{|c|c|c|}
\hline $\begin{array}{l}\text { Dimensionless } \\
\text { group }\end{array}$ & definition & Typical value \\
\hline$h_{0} / R$ & droplet aspect ratio & $0.1-1$ \\
\hline$S r \equiv U t_{f} / R$ & Strouhal number & $1-10^{5}$ \\
\hline$R e \equiv U h_{0} / v_{L}$ & Reynolds number & $<50$ \\
\hline$C a \equiv \mu_{L} U / \sigma$ & Capillary number & $<2 \times 10^{-3}$ \\
\hline$B o \equiv \rho_{L} g h_{0} R / \sigma$ & Bond number & $<0.25$ \\
\hline $\operatorname{Pr} \equiv v_{L} / \alpha_{L}$ & Prandtl number & $>1$ \\
\hline$E \equiv\left(\frac{\alpha_{L}}{U h_{0}}\right)\left(\frac{c_{p, L} \Delta T}{\Delta H_{v a p}}\right)$ & Evaporation number & $<10^{-4}$ \\
\hline $\mathrm{B}_{\mathrm{U}} \equiv \mathrm{g} \beta \Delta \mathrm{Th}_{\mathrm{o}}{ }^{2} \mathrm{t}_{\mathrm{f}} / v_{\mathrm{L}} \mathrm{R}$ & Bouyancy number & $\sim 200$ \\
\hline$M a_{U}=\frac{d \sigma}{d T} \frac{\Delta T_{t} h_{0}}{\mu_{f} R^{2}}$ & Marangoni number & $1-3 \times 10^{4}$ \\
\hline$\beta_{\text {vap }} \Delta T$ & $\begin{array}{c}\text { Dimensionless } \\
\text { temperature } \\
\text { difference }\end{array}$ & $\sim 0.1$ \\
\hline$D_{\text {vap }} t_{f} / R_{0}$ & $\begin{array}{l}\text { Dimensionless vapor } \\
\text { diffusivity }\end{array}$ & $\sim 10^{3}$ \\
\hline$\rho_{\text {vap }} / \rho_{\mathrm{L}}$ & $\begin{array}{l}\text { Ratio of vapor to } \\
\text { liquid densities }\end{array}$ & $<10^{-4}$ \\
\hline$\rho_{\mathrm{G}} / \rho_{\mathrm{L}}$ & $\begin{array}{l}\text { Ratio of gas to } \\
\text { liquid densities }\end{array}$ & $\sim 10^{-3}$ \\
\hline$G r_{G} \equiv\left(\frac{\rho_{v a p}}{\rho_{G}}\right)\left(\frac{g R^{3}}{v_{G}{ }^{2}}\right)$ & $\begin{array}{l}\text { Gas-phase Grashof } \\
\text { number }\end{array}$ & $<3$ \\
\hline $\mathrm{k}_{\mathrm{G}} / \mathrm{k}_{\mathrm{L}}$ & $\begin{array}{l}\text { Ratio of gas to liquid } \\
\text { conductivities }\end{array}$ & $0.04-0.25$ \\
\hline$k_{L} / k_{S}$ & $\begin{array}{l}\text { Ratio of liquid-to-substrate } \\
\text { thermal conductivities }\end{array}$ & $0.1-10$ \\
\hline$k_{L} h_{S} / k_{S} h_{0}$ & $\begin{array}{l}\text { Ratio of substrate-to-liquid } \\
\text { thermal resistivities }\end{array}$ & $0.01-100$ \\
\hline$h_{S}^{2} / \alpha_{S} t_{f}$ & $\begin{array}{l}\text { Ratio of substrate thermal } \\
\text { equilibration time to } \\
\text { droplet drying time }\end{array}$ & $10^{-6}-1$ \\
\hline
\end{tabular}

$E=\frac{2}{S r}\left(\frac{\rho_{\text {vap }}}{\rho_{L}}\right)\left(\frac{D_{\text {vap }} t_{f}}{R h_{0}}\right) \frac{h_{0}}{R}$, showing explicitly how $E$ is obtained from other dimensionless groups through solution of the heat equation. Of course, in general, all equations are coupled, and the solutions for the three dependent groups must be obtained from a simultaneous solution of the coupled momentum, heat, and mass transport equations.

We recap by noting that we obtain five dimensionless groups by considering the geometry, drying time, and viscous properties of the droplet fluid, and another five groups by considering thermal effects in the liquid phase. Five additional groups emerge from the gas and vapor properties, and three groups from considering the thermal properties of the substrate. This gives 18 continuum dimensionless groups governing transport, with two or three relationships connecting them, depending on whether or not a temperature difference is imposed across the droplet. These 18 groups are listed in Table 2, along with typical values of them for small droplets under conditions considered here. The 23 independent parameters used to define these groups are given in Table 3, where the five units of time, length, mass, energy, and temperature allow the 18 given groups to be uniquely defined from the 23 parameters. Other parameters could enter, of course, depending on the physical situation. For example, if the substrate has a radius $R_{S}$ not much larger than that of the droplet, or if a portion of the substrate of radius $R_{s}$, concentric with the droplet, is heated, then the group $R_{s} / R$ enters the analysis, as discussed briefly later. Additional quantities also enter if the droplet contains two volatile liquids or gas is blown across the droplet, for example.

Even if no other continuum level quantities enter the problem, there are molecular or microscopic variables that can play a role in some cases. For example, if the contact line is pinned, the fluid near the contact line must slip along the solid substrate, and this slip is governed by a constitutive equation that contains parameters such as the slip length, which is typically of order molecular size. The ratio of this to the droplet radius is a dimensionless group whose small (but non-zero) value can make results insensitive to its exact magnitude. If the contact line moves, there is another constitutive equation describing this, again with a length parameter governed by microscopic or molecular quantities. ${ }^{18}$ The evaporation might be kinetically controlled under some circumstances, and the kinetic energy of the vapor molecules and their mean free path in the vapor can then play a role in the rate of evaporation. The wetting characteristics of the liquid with the substrate can introduce

Table 3. Definition of 23 Parameters Required for Defining Dimensionless Groups

\begin{tabular}{|c|c|c|}
\hline Parameter & Definition & Typical Values \\
\hline$c_{p, L}$ & heat capacity of liquid & $2-4 \mathrm{~J} / \mathrm{gmK}$ \\
\hline $\mathrm{D}_{\text {vap }}$ & vapor diffusivity & 0.05 to $0.3 \mathrm{~cm}^{2} / \mathrm{s}$ \\
\hline g & gravitational constant & $980 \mathrm{~cm} / \mathrm{s}^{2}$ \\
\hline $\mathrm{h}_{0}$ & droplet height at start of drying & $0.1-1 \mathrm{~mm}$ \\
\hline $\mathrm{h}_{\mathrm{S}}$ & thickness of substrate & $0.1-10 \mathrm{~mm}$ \\
\hline $\mathrm{k}_{\mathrm{G}}$ & thermal conductivity of gas & $0.024 \mathrm{~W} / \mathrm{mK}$ \\
\hline $\mathrm{k}_{\mathrm{L}}$ & thermal conductivity of liquid & $0.1-0.6 \mathrm{~W} / \mathrm{mK}$ \\
\hline $\mathrm{k}_{\mathrm{S}}$ & thermal conductivity of substrate & $0.05-1 \mathrm{~W} / \mathrm{mK}$ \\
\hline $\mathrm{R}$ & droplet radius at start of drying & $\sim 1 \mathrm{~mm}$ \\
\hline$t_{f}$ & drying time & $200-10,000 \mathrm{~s}$ \\
\hline $\mathrm{U}$ & characteristic velocity in droplet & $10^{-3}-10 \mathrm{~mm} / \mathrm{s}$ \\
\hline$\alpha_{S} \equiv k_{S} /\left(c_{p, S} \rho_{S}\right)$ & thermal diffusivity of substrate & $\sim 5 \times 10^{-3} \mathrm{~cm}^{2} / \mathrm{s}$ \\
\hline$\beta \equiv-d\left(\ln \rho_{L}\right) / d T$ & liquid thermal expansion coefficient & $\sim 2 \times 10^{-4 \circ} \mathrm{C}^{-1}$ \\
\hline$\beta_{\text {vap }} \equiv d\left(\ln \rho_{\text {vap }}\right) / d T$ & temperature coefficient of equilibrium vapor density & $0.05-0.1^{\circ} \mathrm{C}^{-1}$ \\
\hline$\Delta \mathrm{H}_{\text {vap }}$ & latent heat of evaporation per unit mass & $\sim 2 \times 10^{6} \mathrm{~J} / \mathrm{gm}$ \\
\hline$\Delta \mathrm{T}$ & temperature difference across droplet & $0.1-4^{\circ} \mathrm{C}$ \\
\hline$v_{\mathrm{G}}$ & kinematic viscosity of gas phase & $0.17 \mathrm{~cm}^{2} / \mathrm{s}$ \\
\hline$v_{\mathrm{L}}$ & kinematic viscosity of droplet liquid & $0.5 \times 10^{-2}-5 \times 10^{-2} \mathrm{~cm}^{2} / \mathrm{s}$ \\
\hline$\rho_{\mathrm{G}}$ & mass density of gas phase & $1.2 \times 10^{-3} \mathrm{gm} / \mathrm{cm}^{3}$ \\
\hline$\rho_{\mathrm{L}}$ & mass density of droplet liquid & $\sim 1 \mathrm{gm} / \mathrm{cm}^{3}$ \\
\hline$\rho_{\text {vap }}$ & mass density of vapor & $2 \times 10^{-6}-10^{-4} \mathrm{gm} / \mathrm{cm}^{3}$ \\
\hline$\sigma$ & surface tension of droplet & $20-70 \mathrm{dyn} / \mathrm{cm}$ \\
\hline $\mathrm{d} \sigma / \mathrm{dT}$ & temperature coefficient of surface tension & $-0.2 \mathrm{dyn} / \mathrm{cm}^{\circ} \mathrm{C}$ \\
\hline
\end{tabular}


parameters such as the Hamaker constant that control transport in thin films near the contact line. Finally, if particles, polymers, crystals, or other deposits are left by the drying droplets, then additional parameters describing their physicochemical and transport characteristics will appear. One of the more important of these is the dimensionless solute diffusivity $D t_{f}$ ' $R^{2}$, which characterizes the ability of the solute with diffusion coefficient $D$ to diffuse across the droplet during the time of evaporation. Except possibly for small molecule solutes, this group is typically small for millimeter size droplets, so that the solute cannot maintain uniform concentration during drying. Other possible dimensionless groups involving solute properties will not be included here, since just the continuum dimensionless groups already present a daunting list. Fortunately, many dimensionless groups can be neglected in various cases. In the sections that follow, I assess the magnitudes of these dimensionless groups for the "typical" droplet parameters discussed in the second section.

Before presenting the "typical" values of the various groups, it is important to emphasize yet again that the massloss velocity, $U_{M L} \sim R / t_{f}<5 \times 10^{-4} \mathrm{~cm} / \mathrm{s}$, does not account for thermally-driven flows, such as Marangoni flows. We will soon see that Marangoni effects can be expected to produce velocities as much as $10^{4}$ times higher than this, depending on the conditions of heat transfer. Because of this, in what follows I will include the effect of Marangoni number $M a_{U}$ in parentheses, in estimates of the dimensionless groups that involve fluid velocity. Since the Marangoni number $M a_{U}$ as defined earlier is the ratio of the magnitude of the expected Marangoni velocity to the mass-loss velocity, if Marangoni stresses are small or absent, then the correct value of the dimensionless group is obtained by simply dropping $M a_{U}$ or replacing it by unity in the following equations.

\section{Dimensionless Groups Controlling Droplet Shape Capillary Number $-\mathrm{Ca} \equiv \frac{\mu_{L} U}{\sigma}<2 \times 10^{-7}\left(\times \mathrm{Ma}_{\mathrm{U}}\right)$}

This tiny value of the capillary number means that the flow in the droplet cannot influence the droplet shape and the shape will be set by interfacial tension, and possibly gravity. Even the presence of thermally-driven flows, such as Marangoni flows, is not likely to increase the capillary number by the sixor seven-orders of magnitude needed to change this conclusion, until the droplet becomes very flat. This exception for flatness derives from the very low-interfacial curvature that results if $h_{0} / R$ is very small, and the large velocity gradients, and consequently high viscous stresses, that result when the film thickness gets very small. The large velocity gradient results from the requirement that the velocity near the free surface $U$, must decrease to zero at the substrate, due to the noslip condition. As a result of these considerations, the stress from viscous flow becomes comparable to that from capillarity when the droplet height-to-radius ratio decreases to $h_{0} / R \sim$ $\mathrm{Ca}^{1 / 3}$. For "typical" droplets considered here, this condition is realized only when the droplet reaches a height of a few microns, by which time the complex physics of dewetting typically overwhelm the macroscopic fluid mechanics.

Bond number - Bo $\equiv \frac{\rho_{L} g h_{0} R}{\sigma}<0.25$

Here $g=980 \mathrm{~cm}^{2} / \mathrm{s}$ is the acceleration of gravity. This aforementioned formula is obtained as the ratio of the capillary pressure $\sigma / R$ to the gravitational pressure head $\rho_{L} g h_{0}$. The Bond number is less than unity for droplets of size $\mathrm{R}=1 \mathrm{~mm}$, but Bo is close enough to unity that some gravitational distortion of the interface away from that of a spherical cap should be expected. For droplets much larger than $1 \mathrm{~mm}$ in radius, droplet heights will be limited by gravity to a capillary height of around $h_{\text {cap }} \approx \sqrt{\sigma / \rho_{L} g}=1-2 \mathrm{~mm}$ or so. Droplets whose volume greatly exceeds $1 \mu \mathrm{L}$ will, therefore, spread into pancakeshaped droplets, or puddles, of height 1-2 mm.

\section{Dimensionless Groups Controlling Momentum Transport}

Dimensionless momentum equilibration time $\frac{t_{\text {mom }}}{t_{f}}=\frac{R e}{S r} \frac{h_{0}}{R} \approx 5 \frac{D_{\text {vap }}}{v_{L}} \frac{h_{0}}{R} \frac{\rho_{\text {vap }}}{\rho_{L}}<0.01$

When the above dimensionless group is small, the velocity field in the droplet can reach a quasi-steady state well before the completion of drying. "Quasi-steady state" means that the velocity field at a given point in time can be obtained by applying a steady-state momentum balance; that is, the time derivative of the velocity can be dropped from the Navier-Stokes equations. Momentum transfer, or velocity, within the droplet can reach a steady state in a time of around $t_{\text {mom }} \sim h_{0}^{2} / v_{L}$, where $v_{L}$ is the kinematic viscosity. Combining this with the estimate for the drying time of $t_{f} \sim 0.2\left(\rho_{L} / \rho_{\text {vap }}\right) R h_{0} / D_{\text {vap }}$, for $t_{f}$ gives $t_{\text {mom }} / t_{f} \sim\left(\rho_{\text {vap }} / \rho_{L}\right)\left(h_{0} / R\right)\left(D_{\text {vap }} / v_{L}\right)$, which is typically much less than unity, because of the very small value of $\rho_{\text {vap }} / \rho_{L}<10^{-4}$. Hence, $t_{\text {mom }}$ is small compared to the drying time $t_{f}$, and we may neglect the momentum equilibration, and drop the time derivative in the Navier-Stokes equation. Another way to obtain this result is to note that the transient term in the Navier-Stokes equation is small compared to the viscous term when the ratio $\mathrm{Re} / \mathrm{Sr}$ is small, where $\operatorname{Re} \equiv \rho_{L} U R /$ $\mu_{L}$ is the Reynolds number and $\mathrm{Sr}$ is the Strouhal number. $\mathrm{Sr}$ is unity when $U \approx U_{M L}=R / t_{\mathrm{f}}$, so that flow is only driven by mass loss (with no thermally-driven flows), and $U$ is greater than this when thermal effects drive flows. However, the ratio $\mathrm{Re} / \mathrm{Sr}$ is independent of velocity and so can remain small whether or not Marangoni flow is present. Thus, momentum transfer comes to a quasi-steady state well before completion of drying and a quasi-steady state in the momentum balance can be assumed for most of the drying process. This quasisteady can be a linear Stokes flow, if the Reynolds number is small, or a nonlinear flow, if it is large.

\section{Liquid-phase convective vs. diffusive momentum transfer: Reynolds number $R e \equiv \frac{\rho_{L} U R}{\mu_{L}}<5 \times 10^{-3}$ $\left(\times M a_{U}\right)$}

The Reynolds number governs the ratio of momentum convection to momentum diffusion and is small for our "typical conditions" unless there is strong Marangoni flow. Since we have seen that momentum transfer quickly attains quasi-steady state relative to the drying time, if $\mathrm{Re}$ is small, the momentum balance equation reduces to the linear Stokes equation. If there is a dominance of diffusive over convective transport for all three forms of transport- mass, heat, and momentum-then the equations for vapor concentration, temperature, and velocity, are all linear, can be solved readily, and typically produce stable, steady, transport. Note also, however, that strong thermally-generated flows, such as Marangoni flows, may increase the velocity enough to bring the Reynolds number above unity, if $M a_{U}>200$, which should not be uncommon, as discussed in the next section. Once convective terms become important in any of the transport equations, the equations become nonlinear, and instabilities may arise. 


\section{Dimensionless Groups Controlling Thermal Transport}

Buoyancy number $-B_{U} \equiv G r_{L} \frac{h_{0}^{2}}{R^{4}} t_{f} v_{L} \approx 0.4 \beta \rho_{L}^{2} g h_{0}^{4}$ $\frac{\Delta H_{\text {vap }}}{k_{L} \mu_{L} R} \approx 200$

Temperature variations in the droplet lead to relative density variations of magnitude $\Delta \rho_{L} / \rho_{L} \approx \beta \Delta T$, where $\beta$ is the thermal expansion coefficient, which is the fractional increase in liquid volume per degree of temperature rise. For typical smallmolecule liquids near room temperature, $\beta \approx 2 \times 10^{-4 \circ} \mathrm{C}^{-1}$. The stress created by this density difference is then of order $g \beta \rho_{L} \Delta T h_{o}$, which should produce a characteristic buoyancydriven velocity of around $g \beta \rho_{L} \Delta T h_{o}{ }^{2} / \mu_{L}$. The ratio $\mathrm{B}_{U}$ of this buoyancy-driven velocity to the mass-loss velocity $U_{M L}=R / t_{f}$ is then given by $\mathrm{B}_{U} \equiv g \beta \rho_{L} \quad \Delta T h_{o}{ }^{2} t_{f} / \mu_{L} R=g \beta \Delta T h_{o}{ }^{2} t_{f} / v_{L} R$. Defining a liquid-phase Grashof number as $G r_{L} \equiv \beta \Delta T g R^{3} / v_{L}{ }^{2}$ (analogous to the gas-phase Grashof number), we obtain the value for $B_{U}$ given above: $B_{U} \equiv \mathrm{Gr}_{L} \frac{h_{0}^{2}}{R^{4}} t_{f} v_{L}$. Once the values for $t_{f}$ and $\Delta T$ from Eqs. 2 and 4 are substituted into this expression, we find that $B_{U}$ does not depend on the volatility of the liquid and so should exceed around 100 for most liquids for droplets of radius $1 \mathrm{~mm}$ and height around $0.5 \mathrm{~mm}$. This would seem to imply that thermal buoyancy effects are typically large enough to contribute appreciably to the flow in the droplet. However, thermally-induced Bouyancy effects are usually exceeded by an even larger effect that of thermally-induced interfacial-tension driven flows, considered next.

\section{Marangoni Number $-M a_{U} \equiv-\frac{d \sigma}{d T} \frac{\Delta T t_{f} h_{0}}{\mu_{L} R^{2}}=$ $-0.4 \frac{d \sigma}{d T} \frac{\Delta H_{\text {vap }} h_{0}{ }^{3}}{k_{L} v_{L} R^{2}} \approx 3 \times 10^{3}$ to $3 \times 10^{4_{L}}$}

Temperature variations in the droplet resulting from latent heat of evaporation lead to surface tension variations, governed by the temperature coefficient of surface tension, $d \sigma / d T$, which is around $-0.2 \mathrm{dyn} / \mathrm{cm}^{\circ} \mathrm{C}\left(-0.166 \mathrm{dyn} / \mathrm{cm}^{\circ} \mathrm{C}\right.$ for water near room temperature). The product $-(d \sigma / d T) \Delta T$ is the magnitude of the surface tension difference across the droplet, where $\Delta T$ is the maximum temperature difference along the droplet free surface, usually the difference between the temperature at the top (or center) of the droplet, $T_{c}$ and that at the edge, or contact line $T_{e}$, so that $\Delta T=\mid \mathrm{T}_{\mathrm{c}}-\mathrm{T}_{\mathrm{e}} \mathrm{l}$. The temperature coefficient of surface tension $d \sigma / d T$, is usually negative. The gradient in surface tension is then $-(d \sigma / d T) \Delta T / R$, and this surface stress drives a velocity gradient, whose magnitude is of order the stress divided by viscosity, or $-(d \sigma / d T) \Delta T /\left(R \mu_{L}\right)$. The Marangoni velocity is then the droplet height times this gradient, or $U_{M a r} \sim-(d \sigma / d T) \Delta T h_{0} /\left(\mu_{L} R\right)$. The ratio of the Marangoni velocity to the velocity driven by mass loss $U_{M L}=R / t_{f}$, gives a Marangoni number of $M a_{U} \equiv-(d \sigma / d T) \Delta T t_{f} h_{0} /\left(\mu_{L} R^{2}\right)$. This is an appropriate Marangoni number for determining the importance of Marangoni flow relative to the mass-loss velocity $U_{M L}$. This definition of Marangoni number differs from a more common choice $M a \equiv \frac{d \sigma}{d T} \Delta T h_{0} / \mu_{L} \alpha_{L}$, discussed in the following. Using $\mathrm{Ma}_{U} \equiv-(d \sigma / d T) \Delta T t_{f} h_{0} /\left(\mu_{L} R^{2}\right)$ and $t_{f}$ $\approx 0.2\left(\rho_{L} / \rho_{\text {vap }}\right) R h_{0} / D_{\text {vap }}$ from Eq. 2 , along with an expression for $\Delta T \approx 2\left(h_{0} / R\right) \Delta H_{\text {vap }} D_{\text {vap }} \rho_{\text {vap }} / k_{L}$ from Eq. 4, yields $M a_{U}=-0.4 \frac{d \sigma}{d T} \frac{\Delta H_{\text {vap }} h_{0}{ }^{3}}{k_{L} v_{L} R^{2}}$. Using values from Table 1, in cgs units, we find that $M a_{U}$ is between around $0.5 \times 10^{6}\left(h_{0}{ }^{3} / R^{2}\right)$ for hexanol and $5 \times 10^{6}\left(h_{0}^{3} / R^{2}\right)$ for ethanol. So, for droplets of fluids similar to those in Table 1 with droplet height around $0.4 \mathrm{~mm}$ and radius $1 \mathrm{~mm}$, the Marangoni number $M a_{U}$ ranges from 3000 to 30,000 . This value will drop rapidly as the droplet evaporates and $h_{0}$ decreases if $R$ is fixed (pinned contact line). Like

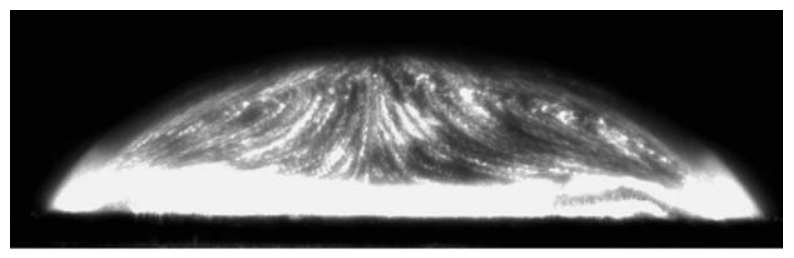

a

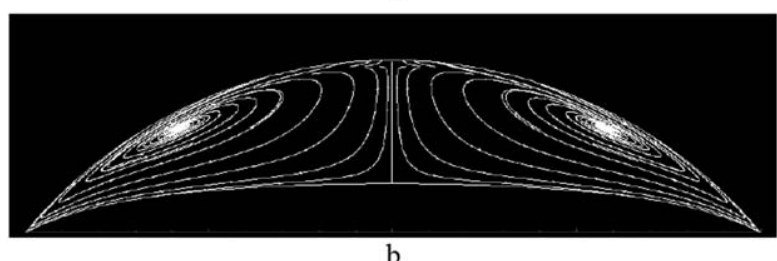

Figure 5. Marangoni flow in a drying octane droplet from (a) experimental imaging, and (b) the lubrication solution.

The prediction in (b) includes the optical "spherical lens" distortion produced by the difference in index of refraction between the octane fluid and air. Reprinted with permission from Hu and Larson, ${ }^{17} J$ Phys Chem B; 110:70907094, 2006. Copyright 2006 American Chemical Society.

the buoyancy number, $M a_{U}$ is rather insensitive to fluid volatility. The large value of this number means that the Marangoni flows will ordinarily be much larger than the mass loss velocity $U_{M L}=R / t_{f}$. We, thus, find that thermal effects, producing buoyancy and especially surface-tension gradients, should strongly influence the velocity field in the droplet. From the aforementioned estimates, one might estimate that the surface-tension driven flow should be roughly 100 times larger than the buoyancy-driven flow, and one might, therefore, expect to be able to neglect the buoyancy-driven flow. However, a more detailed analysis and experiments (discussed later) show that actual Marangoni velocities are around 30-fold lower than the crude estimate given earlier, and can be comparable to buoyancy-driven velocities. In fact, we shall soon see that the thermally-driven velocities are large enough that the fluid can transport heat much faster than occurs by thermal diffusion, and this then attenuates the driving force for thermally-driven flow.

If thermal convection can be neglected (true only under conditions discussed later), then Marangoni effects can easily be incorporated into the lubrication analysis, simply by using the Marangoni stress, $\tau_{z r}=d \sigma / d r$ in the boundary condition for the shear stress at the free surface, rather than the no-shearstress boundary condition $\tau_{z r}=0$ at the free surface of the droplet. Here $\tau_{z r}$ is the shear stress, and $\sigma$ is the surface tension. The surface tension becomes dependent on radial location along the free surface because it is temperature sensitive, and temperature varies along the free surface because of uneven evaporative cooling and uneven heat transport to the free surface from the underlying fluid and substrate. Including the Marangoni boundary condition yields the following extra terms in the velocity field

$$
\tilde{u}_{r}=\frac{1}{4} M a_{U} \tilde{h} \frac{d \tilde{T}}{d \tilde{r}}\left(2 \frac{\tilde{z}}{\tilde{h}}-3 \frac{\tilde{z}^{2}}{\tilde{h}^{2}}\right)
$$

and

$$
\tilde{u}_{z}=-\frac{1}{4} M a_{U}\left(\tilde{z}^{2}-\frac{\tilde{z}^{3}}{\tilde{h}}\right)\left(\frac{d^{2} \tilde{T}}{d \tilde{r}^{2}}+\frac{1}{\tilde{r}} \frac{d \tilde{T}}{d \tilde{r}}\right)-\frac{1}{4} M a_{U}\left(\frac{\tilde{z}^{3}}{\tilde{h}^{2}}\right) \frac{d \tilde{T}}{d \tilde{r}} \frac{\partial \tilde{h}}{\partial \tilde{r}}
$$

where $\tilde{T} \equiv \frac{T-T_{c}}{T_{e}-T_{c}}$. These extra terms, which add linearly to the velocities given by Eqs. 10 and 11, typically so dominate 

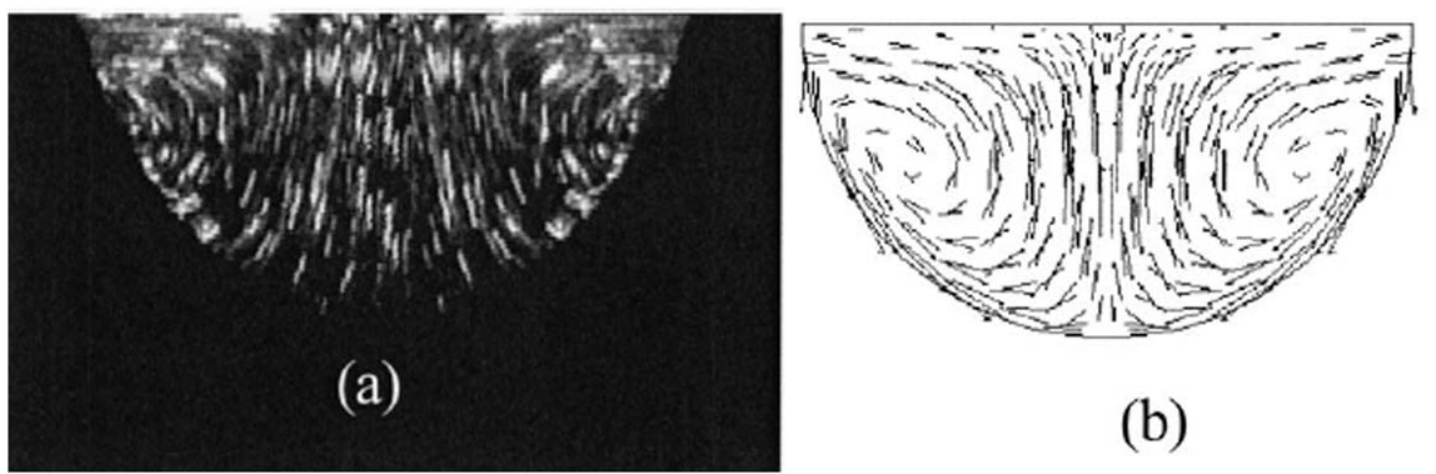

(b)

Figure 6. (a) Experimental streak lines and (b) numerical velocity field for a droplet of $n$-octane evaporating at ambient temperature.

Dimensionless groups: $\mathrm{Ma}=5.73 \times 10^{3}, \mathrm{Gr}_{L}=300$. In the vapor phase, the Grashof number $\mathrm{Gr}_{G}$ produced by the temperature variation was unity, and that produced by the octane/air composition variation was around 20 (Reprinted with permission from Savino and Fico, ${ }^{21}$ Phys Fluids; 16:3738-3754, 2004. Copyright 2004, AIP Publishing LLC).

the flow that Eqs. 12 and 13 provide, to a first approximation the entire flow field. An example flow dominated by the Marangoni contributions is shown in Figure 5a. The corresponding simulated flow field in Figure $5 \mathrm{~b}$ was derived by neglecting the effect that fluid convection has on the temperature profile within the droplet, which in Figure $5 \mathrm{~b}$ is taken from the solution to the heat diffusion equation (a Laplace equation), without the heat convection term. The neglect of heat convection is reasonable only if the dimensionless group $\operatorname{RePr}$ is small, and when $M a_{U}$ is large, convection of heat is likely to become important, as discussed later.

A careful study of Marangoni and buoyancy-driven convection in drying hydrocarbon droplets was carried out by Savino and Fico. ${ }^{21}$ The droplets were hung upside down from a metallic substrate of $2.5 \mathrm{~mm}$ radius, equal to the radius $\mathrm{R}$ of the hanging droplet, which was comparable to the droplet height $h_{0}$; see Figure 6 . The temperature field within the droplet was measured by an infrared camera and velocities were measured by the length of streaks formed by small suspended particles imaged over a fixed time periods. Results were compared to the predictions of a finite volume numerical analysis, which included thermal and convection effects both within the droplet and in the surrounding air. Reasonable agreement was obtained between predictions and measurements. For the octane droplet hanging from a surface

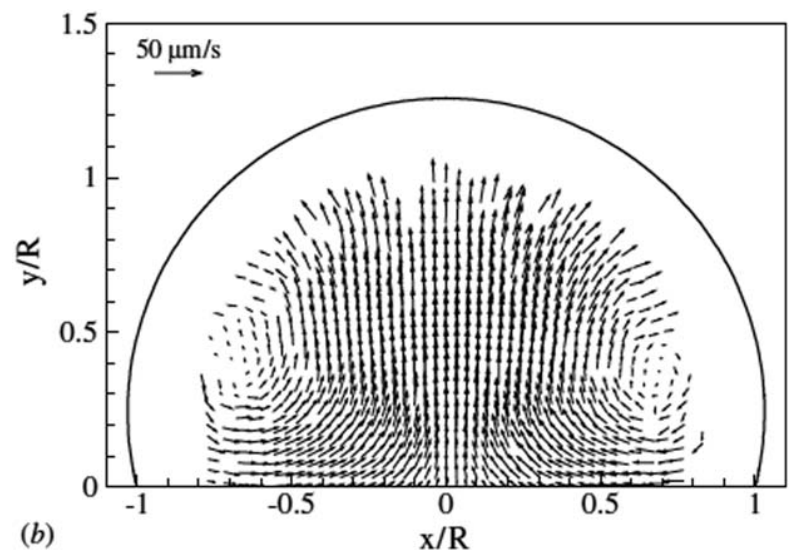

Figure 7. Instantaneous velocity field measured by particle imaging velocimetry inside a water-ethanol droplet with $5 \%$ ethanol concentration.

The flow field has been corrected for optical distortion due to the spherical lens effect (from Kang et al. ${ }^{22}$ ). at ambient temperature, an evaporation-induced temperature difference of $\Delta T=1.3 \mathrm{C}$ was measured along the droplet surface, compared to a predicted difference of $1.2 \mathrm{C}$. The average upward velocity along the axis of the droplet was measured to be $8 \mathrm{~mm} / \mathrm{s}$, not far from the predicted value of $6.3 \mathrm{~mm} / \mathrm{s}$. From the simulations, it was determined that in the absence of buoyancy, this velocity would have been only $3.5 \mathrm{~mm} / \mathrm{s}$, which is the Marangoni contribution to the velocity. The Marangoni number for this flow was computed to be $M a \equiv \frac{d \sigma}{d T} \frac{\Delta T R}{\mu_{L} \alpha_{L}}$. Using the scaling analysis described earlier, the characteristic Marangoni velocity is estimated to be $U_{M a r} \approx-(d \sigma / d T) \Delta T h_{0} /\left(R \mu_{L}\right)=\operatorname{Ma} \alpha_{\mathrm{L}} \mathrm{h}_{0} / \mathrm{R}^{2} \approx 180 \mathrm{~mm} / \mathrm{s}$, 30 times larger than given by the full numerical analysis! The liquid phase Grashof number $G r_{L} \equiv \beta \Delta T g R^{3} / v_{L}^{2}$ was determined to be 300 for the octane droplet. An estimate of the buoyancy-driven velocity, from the arguments in the previous section, is $U_{\text {bouy }} \sim G r_{L} \frac{h_{0}^{2}}{R^{3}} v_{L} \approx 60 \mathrm{~mm} / \mathrm{s}$, which is also more than 10 -fold higher than predicted by the full numerical analysis. Thus, the rough scaling formulas give velocities that are about an order of magnitude of higher than the more accurate predictions from full numerical analysis for both Marangoni and buoyancy-drive flows.

Much of this difference is no doubt due to reduction of temperature gradients by fluid mixing via thermal convection, neglected in the simple scaling formulas, and described shortly. Rapid convection within the droplet will transport heat more efficiently than thermal conduction can, and thus limit the temperature differences that drive such flows to begin with. Thus, the negative feedback effects that these flows have on the temperature gradient will be important, and the magnitude of these nonlinear feedbacks needs to be resolved by numerical analysis. Savino and Fico ${ }^{21}$ showed that Marangoni convection can enhance the overall rate of heat transfer from the substrate to an octane droplet by almost $50 \%$ relative to a stagnant droplet. Even more important is the redistribution of heat produced by convection, which will make the droplet temperature more uniform than it would otherwise be. Thus, while calculation of Marangoni flow by the aforementioned simplified lubrication analysis, with its one-way coupling of the temperature field to the flow field, is useful for qualitative estimates of the flow field, quantitative predictions usually require numerical analysis with two-way coupling of thermal and momentum transport.

Savino and Fico ${ }^{21}$ also measured the temperature and flow fields produced in a nonvolatile hanging silicone droplet by 


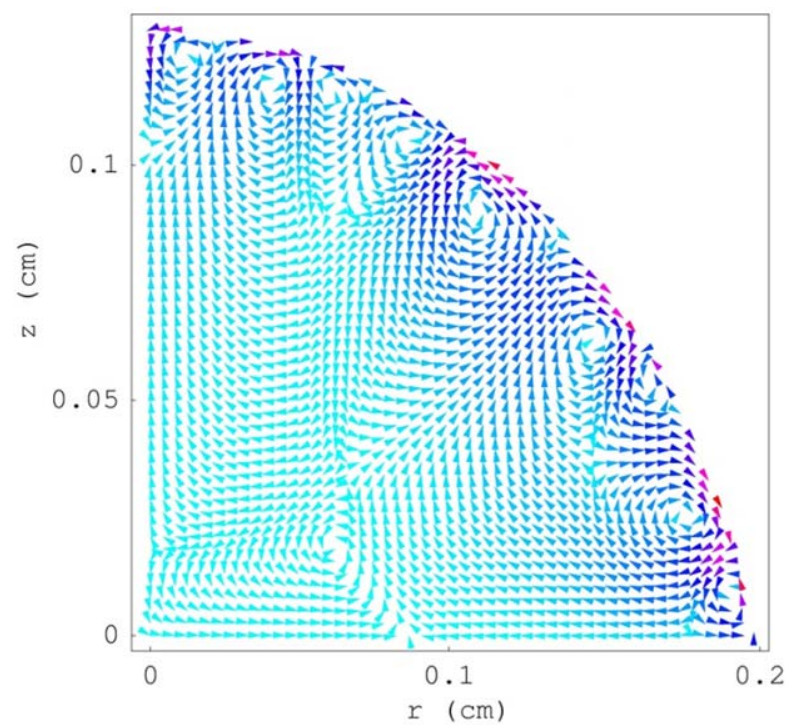

Figure 8. Predicted velocity field $0.16 \mathrm{~s}$ after start of drying of a toluene droplet, $2 \mathrm{~mm}$ in radius and $1.3 \mathrm{~mm}$ high.

For initial conditions, at time $=0$, the droplet was stationary with uniform temperature and no vapor yet present in the surrounding air. The simulations assumed a fixed substrate temperature and solved the vapor phase diffusion equations as well as the full heat and momentum (Navier-Stokes) equations for the liquid (from Barash, Bigioni, Vinokur, and Shchur, ${ }^{27}$ Phys Rev E; 79:046301, 2009. Copyright 2009 by the American Physical Society). [Color figure can be viewed in the online issue, which is available at wileyonlinelibrary.com.]

heating the substrate to $60^{\circ} \mathrm{C}$. A velocity of $8 \mathrm{~mm} / \mathrm{s}$ could be thereby produced in a 5 centistokes fluid. For octane on a substrate heated to $60^{\circ} \mathrm{C}$, where both evaporation and substrate heating drive temperature variations, a velocity of around $50 \mathrm{~mm} / \mathrm{s}$ was obtained, and predicted.

Marangoni flows can also be induced by concentration gradients produced when droplets of mixed solvent evaporate. An example, depicted in Figure 7, is in the evaporation of ethanol-water mixtures. Here, the higher volatility of the ethanol should lead to both density variations within the droplet and surface tension variations along the droplet surface, both of which can produce convective effects. The flow depicted in Figure 7 has not been predicted quantitatively, or even qualitatively, to date. At higher ethanol concentrations $(20 \%$, for example), the flow is observed to become unstable.

Dimensionless heat equilibration time $t_{\text {heat }} / t_{f}=\frac{R e P r}{S r} \frac{h_{0}}{R} \sim 5 \frac{D_{\text {vap }}}{\alpha_{L}} \frac{h_{0}}{R} \frac{\rho_{\text {vap }}}{\rho_{L}}<0.1$

Arguments similar to those used for momentum transfer in the previous section can be applied to heat transfer within the droplet, giving a heat equilibration time of $t_{\text {heat }} \sim h_{0}{ }^{2} / \alpha_{L}$, where $\alpha_{L}$ is the thermal diffusivity of the liquid, which as mentioned earlier is around $10^{-3} \mathrm{~cm}^{2} / \mathrm{s}$ for organic liquids and roughly twice as large as this for water. The argument is equivalent to that for momentum transfer, with $\alpha_{\mathrm{L}}$ replacing $v_{L}$. Thus the ratio $t_{\text {heat }} / t_{f}$ exceeds $t_{\text {mom }} / t_{f}$ by a factor of the Prandtl number $\operatorname{Pr}=v_{L} / \alpha_{L}$. The ratio $t_{\text {heat }} / t_{f} \sim 5\left(D_{\text {vap }} / \alpha_{L}\right)$ $\left(h_{0} / R\right) \rho_{\text {vap }} / \rho_{L}$ readily follows as the criterion for attainment of a quasi-steady state. Again, because of the small value of this ratio, we expect thermal equilibration to be faster than the drying process, but less so then for momentum equilibration, because the Prandtl number is usually greater than unity for liquids ( $\mathrm{Pr}$ is around 7 for water near room temperature). As we will see later, thermal equilibration is much less rapid than equilibration of the vapor concentration field just above the droplet, since the ratio $D_{\text {vap }} / \alpha_{L} \approx 10^{2}$ is very large.

The aforementioned criterion for attaining equilibration of heat diffusion assumes that the substrate on which the droplet rests has high-thermal conductivity and does not itself take significant time to thermally equilibrate. If the substrate also needs to equilibrate thermally, it will take even longer for heat diffusion to reach a quasi-steady condition. The substrate thermal equilibration time can be estimated to be $t_{\text {sub- }}$ strate $\sim h_{S}{ }^{2} / \alpha_{S}$, where $\alpha_{S} \equiv k_{S} /\left(c_{p, S} \rho_{S}\right)$ is the thermal diffusivity of the substrate and $h_{S}$ is the substrate thickness. Here, $c_{p S}$ is the heat capacity of the substrate, $k_{S}$ its thermal conductivity, $\rho_{S}$ and its density. For thick substrates, the substrate thermal equilibration time becomes large, and if it is as large as or larger than the droplet drying time $t_{f}$, the droplet will not reach a quasi-steady-state before it has completely evaporated. Ristenpart et al. were able to show that for very thick substrates (effectively infinitely thick), the gradients of temperature nevertheless can reach a steady state near the contact line. However, in that case, the overall temperature of the droplet away from the contact line will continue to decrease, as thermal energy is withdrawn from the infinite heat reservoir in the substrate, unless heat conduction from the gas finally supplies the heat needed to offset the loss due to latent heat of evaporation. Transport from the gas will compete with that from the substrate if $k_{G} / k_{S}$ is not too small. In any event, of the three transport processes- mass, heat, and momentum - heat equilibration is typically the slowest, even if heat conduction in the substrate is instantaneous, and so heat transfer will create the longest transients. We will discuss some of the effects of time-dependent thermal equilibration later.

Even when $t_{\text {heat }} / t_{f}$ is small, at early times much less than $t_{f}$, heat has not yet had time to diffuse throughout the droplet, and, thus, in the early stages of evaporation there can be multiple, transient, Marangoni vortices. These are depicted in Figure 8, which is taken from a simulation of a drying toluene droplet, whose thermal and hydrodynamic properties are close to those of ethanol. This simulated droplet, $2 \mathrm{~mm}$ in radius and $1.3 \mathrm{~mm}$ high, dries out in around $500 \mathrm{~s}$, but in the first fraction of a second, up to $0.3 \mathrm{~s}$, the surface of the drying droplet is roiled by multiple vortices, produced by a Marangoni (surface-tension driven) instability, similar to the one produced on flat layers of volatile liquid. The condition for such a Marangoni instability on a flat fluid layer is that the Marangoni number, defined in this case by Ma $\equiv \frac{d \sigma}{d T} \Delta T h_{0} / \mu_{L} \alpha_{L}$, exceed 83 . Here $\Delta T$ is the temperature difference between the substrate (taken to be at constant temperature) and the layer surface, and $d \sigma / d T$ is the temperature coefficient of surface tension. (This Marangoni number differs from the one defined earlier as $M a_{U}$.) In the toluene example depicted in Figure 8, $M a$ is around 2,800, which greatly exceeds the critical value of 83 required for instability to formation of thermocapillary eddies on a flat fluid layer. These eddies have dimensionless wavelength inversely proportional to the square root of the Marangoni number. For infinite flat layers, the multiple eddies persist, but for a drying droplet, with nonuniform thickness, eddies quickly merge to form a single axisymmetric eddy, which persists 
throughout most of the drying time, as discussed earlier. The time required for the small eddies to merge into one large should be roughly the heat diffusion time $t_{\text {heat }}$, but in the simulations depicted in Figure 8, merger of eddies occurs at least 10 times faster than this estimate. Sefiane and coworkers $^{18}$ have also carried out simulations of drying droplets over heated substrates and have shown the formation of multiple vortices, which become more pronounced at higher Prandtl numbers $\operatorname{Pr} \equiv v_{L} / \alpha_{L}$, or, equivalently, higher $t_{\text {heat }} / t_{f}$. In experiments with droplets on heated substrates, Sobac and Brutin ${ }^{25}$ observed "hydrothermal wave" patterns, which could be explained qualitatively by a linear stability analysis. $^{18}$ At high enough Marangoni numbers, $M a>22,000$, the flow can become turbulent. ${ }^{26}$

\section{Liquid-phase convective vs. diffusive heat transfer: $\frac{U h_{0}}{\alpha_{L}}=\operatorname{PrRe}<0.05\left(\times M a_{U}\right)$}

The product of Prandtl number and Reynolds number, PrRe, governs the relative rates of heat convection and heat diffusion, the former being small relative to the latter when $\operatorname{PrRe} \ll 1$. Thus, the two dimensionless groups $t_{\text {heat }} / t_{f}$ discussed previously and PrRe are not independent, but are both controlled by Pr. For our "typical droplet" without Marangoni flow, PrRe is small, and heat convection can be neglected. This allows us to use the Laplace equation for heat conduction to obtain the velocity field throughout the liquid droplet, which was used to obtain the flow field shown in Figure 5b. However, when Marangoni flow becomes important, the convection within the droplet becomes much more vigorous, and heat convection will need to be considered.

Using the estimates of the Marangoni number $\mathrm{Ma}_{U}$ given below, PrRe is in the range of 3 (for hexanol) to around 1500 (for ethanol). This range extends from negligible convective heat transfer to dominant heat transfer. This can be seen in the numerical results shown in Figure 9 from Barash. $^{28}$ The temperature isotherms for hexanol are those for essentially pure conduction, which are nearly parallel lines except near the droplet free surface. However, more volatile liquids show large distortions of the isotherm, owing to the Marangoni recirculation, which carries fluid, and its associated heat, from the edge toward the droplet center, thereby distorting the isotherms. From these isotherms, it is evident that heat convection becomes appreciable once PrRe exceeds around 10 or so. Once this happens, the temperature field is no longer determined by pure conduction and so the Marangoni flow can no longer be expected to follow Eqs. 12 and 13 , even for relatively flat droplets. In this case, the nonlinear coupling between heat transfer and fluid flow necessitates a numerical solution to the combined heat/momentum equations.

Finally, we again emphasize that the thermally-driven velocities will enter some of the dimensionless groups considered above. That is, once the velocity $U$ is estimated with Marangoni and/or buoyancy effects included, the estimated values of $\mathrm{Ca}$, and $\mathrm{Re}$ must be revised by multiplication by the Marangoni number $\mathrm{Ma}_{U}$. We already performed this multiplication above when considering heat convection. The capillary number $\mathrm{Ca}$ is so low $\left(2 \times 10^{-7}\right)$ that even multiplied by $\mathrm{Ma}_{U}$ it remains below unity, so that the sphericalcap shape is likely to be retained even in the presence of Marangoni flow. The Reynolds number Re, however, can be as high as $5 \times 10^{-3}$ even without Marangoni flow. So, for
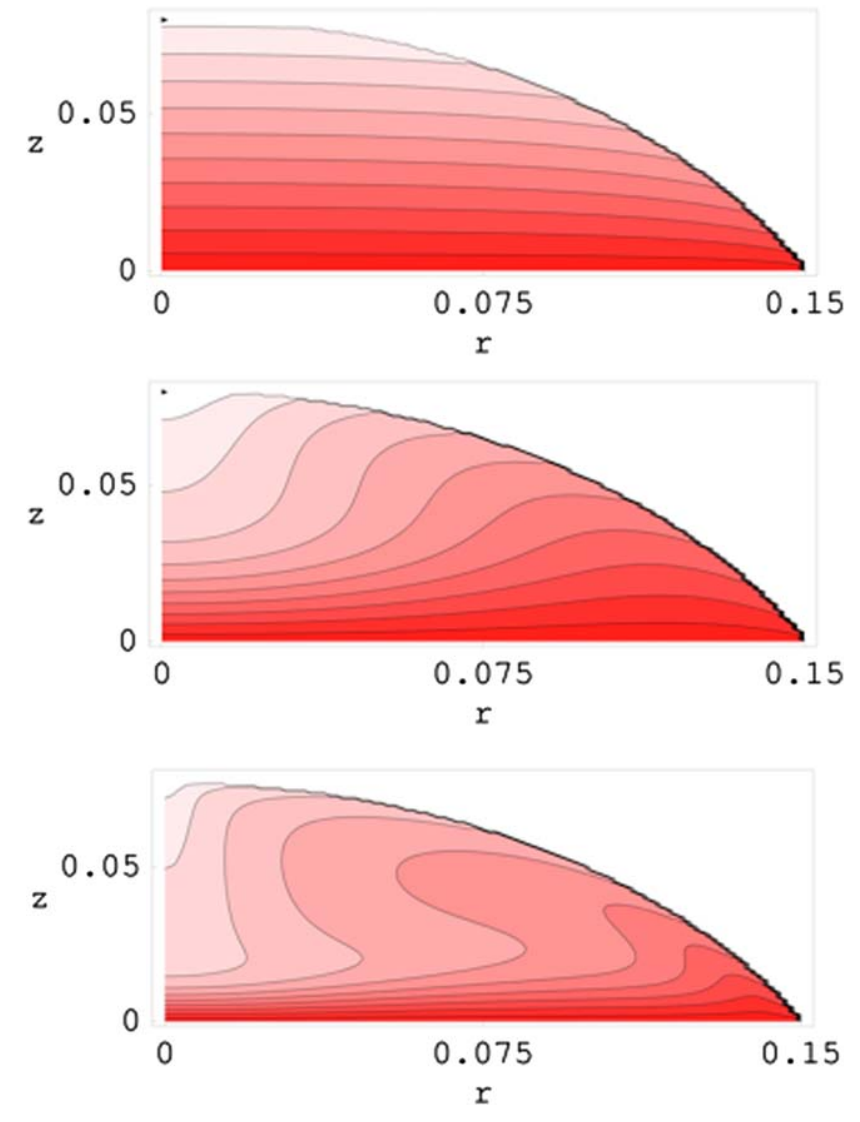

Figure 9. Simulated temperature isotherms for hexanol (top), 1-butanol (middle), and ethanol (bottom). Units of axes are $\mathrm{cm}$ from Barash. ${ }^{28}$

[Color figure can be viewed in the online issue, which is available at wileyonlinelibrary.com.]

strong Marangoni flow, with $\mathrm{Ma}_{U}=3 \times 10^{4}$, inertia may become important, perhaps even important enough to lead to secondary flows or instabilities.

\section{Dimensionless Groups Controlling Gas-Phase Mass and Momentum Transfer}

Dimensionless vapor-phase mass-transfer equilibration time $-t_{\text {vap }} / t_{f} \sim 5 \rho_{\text {vap }} / \rho_{L}<5 \times 10^{-3}$

Since this dimensionless group is small, the vapor concentration above the surface of the droplet reaches a quasisteady state well before the completion of drying. In this context, "quasi-steady state" means that the vapor field above the droplet at a given point in time can be obtained by applying a steady-state mass flux balance; that is, the time derivative of the concentration can be dropped from the mass transport equation. If vapor transport is purely diffusive (i.e., no convection), then the vapor transport equation reduces to Laplace's equation for steady mass transport. The time derivative in principle is needed because the droplet is shrinking with time, and hence the vapor concentration field adjusts continuously to this shrinkage, producing a time rate of change of vapor concentration everywhere. However, if the rate of shrinkage is slow compared to the time required for the vapor field to adjust to that shrinkage, then at each moment in time, a steady-state mass transport equation can 
be solved, using the instantaneous droplet shape as the boundary condition. The validity of this quasi-steady-state approximation is governed by ratio of the characteristic time for the vapor field to adjust to changes in droplet shape $t_{\mathrm{vap}}$, to the characteristic time for droplet shrinkage, which is just the drying time $t_{f}$. The former quantity scales roughly as $t_{\text {vap }} \sim R h_{0} / D_{\text {vap }}$, where $D_{\text {vap }}$ is the diffusivity of the vapor, as defined earlier. As discussed earlier, the drying time then scales as $t_{f} \sim 0.2\left(\rho_{L} / \rho_{\text {vap }}\right) R h_{0} / D_{\text {vap }}$, if the vapor concentration far from the droplet is zero, and is greater than this otherwise. The ratio of these two time constants is just $t_{\text {vap }} / t_{f} \sim 5 \rho_{\text {vap }} / \rho_{L}$, which is very small. Droplets that dry in a time scale of, say $1000 \mathrm{~s}$, will have vapor concentrations fields that equilibrate near the droplet within $1 \mathrm{~s}$ or so. Hence, the quasi-steady-state approximation for mass transfer is normally very well justified, more so than the corresponding approximations for momentum and heat transfer, at least beyond a time $t_{\mathrm{vap}}$, after the droplet has been deposited onto the surface. I should remark, however, that, unlike the small spatial domain $\left(R\right.$ or $\left.h_{0}\right)$ for heat and momentum transport within the droplet, the spatial domain of the vapor field extends to large distances. Hence, while the vapor field within a distance $R$ or $h_{0}$ of the droplet is rapidly driven toward equilibrium, the vapor field far from the droplet will equilibrate much more slowly, over time scales proportional to the square of the distance from the droplet. As a result, even though the vapor field near the droplet quickly comes close to quasi-steady state, there will be small corrections to this vapor field that die out slowly, as $1 / \sqrt{ } t^{27}$

\section{Gas-Phase Convective vs. Diffusive Mass Transfer:}

$$
\text { Grashof Number }-G r_{G} \equiv\left(\frac{\rho_{\text {vap }}}{\rho_{G}}\right)\left(\frac{g R^{3}}{v_{G}^{2}}\right)<3
$$

Here $v_{G}$ is the kinematic viscosity of the air, which is around $0.15 \mathrm{~cm}^{2} / \mathrm{s}$ at room temperature and pressure, and $\rho_{\mathrm{G}}$ is the density of surrounding gas, which is usually air, with a density of roughly $1.3 \times 10^{-3} \mathrm{~g} / \mathrm{cm}^{3}$. This differs in general from the density of the vapor from the droplet, $\rho_{\text {vap }}$, producing a buoyancy effect, which, if large enough, leads to gasphase natural convection. The gas-phase Grashof number controls the onset and strength of this natural convection in the air, which is expected to be weak when $\mathrm{Gr}_{G}<1$. When $\mathrm{Gr}_{G}$ exceeds unity, natural convection will accelerate the rate of evaporation of the droplet, which will then no longer be diffusion-controlled. On the other hand, when $\mathrm{Gr}_{G}<1$, and when vapor mass transfer is quasi-steady, the vapor phase concentration field will satisfy Laplace's equation, a linear equation, at each instant in time. From the aforementioned estimate, $\mathrm{Gr}_{G}$ slightly exceeds unity for ethanol; however, for less volatile liquids natural convection should be weak, unless the droplet radius is larger than $1 \mathrm{~mm}$, in which case $\mathrm{Gr}_{G}$ becomes large, due to the its dependence on the cube of $R$.

If the droplet radius exceeds around $1 \mathrm{~mm}$ or so, so that natural convection is important, the Bond number $B o$ $\equiv \frac{\rho_{L} g h_{0} R}{\sigma}$ will approach or exceed unity, and the droplet surface will no longer be that of a spherical cap. It will instead have a height given by the capillary length $h_{\text {cap }} \approx \sqrt{\sigma / \rho_{L} g}$, which is around $1-2 \mathrm{~mm}$ in terrestrial gravity. If the droplet radius is increased to $10 \mathrm{~mm}$ or more, the "droplet" becomes a puddle or film. The larger radius $\mathrm{R}$ produces a bigger plume of vapor, which makes it more strongly affected by buoyancy, as represented by the larger value of the vapor-

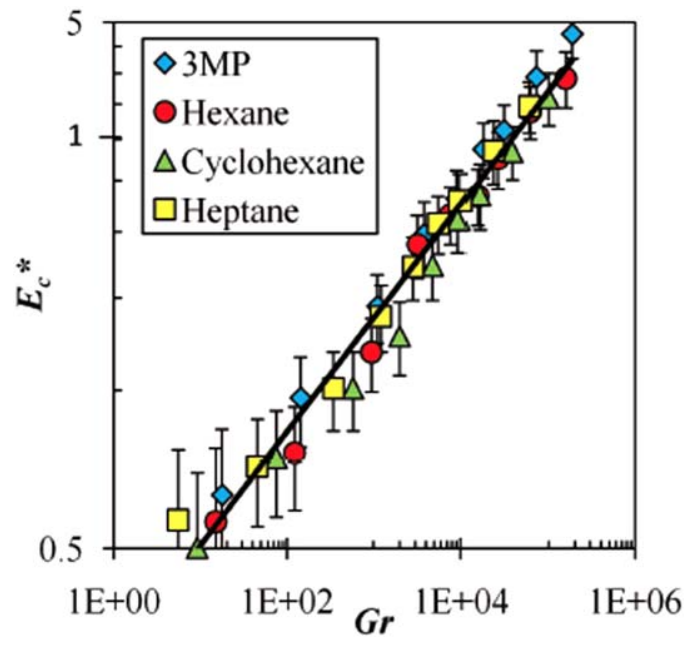

Figure 10. Plot of the dimensionless convective contribution to evaporation as a function of the gas-phase Grashof number.

Here $E_{c}^{*}=E^{*}-1$, where $E^{*}$ is the rate of evaporation from the droplet normalized by the rate of evaporation in the absence of convection. The solid line is the empirical formula (Reprinted from Kelly-Zion, Pursell, Vaidya, and Batra, ${ }^{29}$ Coll Surf A: Physicochem Eng Aspects; 381,1-3:31-36, 2011, with permission from Elsevier). [Color figure can be viewed in the online issue, which is available at wileyonlinelibrary.com.]

phase Grashof number $G r_{G} \equiv\left(\frac{\rho_{\text {vap }}}{\rho_{G}}\right)\left(\frac{g R^{3}}{v_{G}^{2}}\right)$, which is proportional to the cube of the droplet radius. This natural convection enhances the rate of droplet evaporation relative to that produced by pure diffusion. A study by Kelly-Zion et al. ${ }^{29}$ showed that the enhancement of evaporation of liquid drops and puddles of 3-methylpentane, hexane, cyclohexane, and heptane can be represented by the simple equation $E^{*}=1+\operatorname{Gr}_{G}{ }^{0.216}$, as shown in Figure 10 , where $E^{*}$ is the drying rate normalized by the drying rate in the absence of convection.

\section{Interfacial kinetic vs. diffusive limitation to mass}

transfer $P_{\text {vap }} / P<0.01$ or $\frac{D_{\text {vap }} \sqrt{2 \pi R_{\text {gas }} T_{\text {sat }} / M}}{R \alpha \rho_{\text {sat }} \Delta H_{\text {vap }}}<10^{-3}$

If the droplet fluid has modest or low volatility, such that its vapor pressure is much less than the ambient pressure $P$, then when molecules in the droplet escape the liquid into the vapor, they will typically collide very frequently with surrounding gas molecules before they get far from the interface. As a result, they will bounce back and collide with the liquid-vapor interface often enough to have a high probability to recondense into the liquid. Thus, the net rate of evaporation in this case is controlled by the rate at which molecules can diffuse far away from the interface, not the rate at which they escape from the liquid. This condition typifies modestly volatile liquids, with vapor pressures below around $10 \mathrm{kPa}$, slowly drying into air at atmospheric pressure. However, if the ambient pressure is reduced substantially, so that $P$ approaches $P_{\text {vap }}$, then the mean-free path of a vapor molecule, which is the distance traveled between collisions, increases as $1 / \mathrm{P}$, and can reach values of tens of microns for pressures below $1 \mathrm{kPa}$. Since a vapor molecule does not recondense every time it strikes the interface, at low-gas pressure it has a much better chance of permanently escaping the liquid once it's gotten out of the liquid for the 
first time. In this situation, escaping the interface for the first time can become the rate-controlling step for evaporation.

Most studies of droplet drying are carried out at atmospheric pressure of air with only moderately volatile droplets, where vapor-phase diffusion controls the drying rate. In this case, the drying rate is given by Eq. 3. However, Ward and coworkers $^{30-33}$ have studied extensively the case in which the gas pressure is reduced by a vacuum pump to a value comparable to the vapor pressure, that is, down to values below $1 \mathrm{kPa}$ for water, ethanol, and other small-molecule liquids. For such a situation, the vapor diffusivity $D_{\text {vap }}$ approaches $10 \mathrm{~cm}^{2} / \mathrm{s}$, and evaporative fluxes predicted by Eq. 3 exceed by a factor of 100 those obtained at atmospheric pressure. It is found, in these cases, that the measured evaporation fluxes of around 0.05 to $0.3 \times 10^{-4} \mathrm{~g} / \mathrm{cm}^{2} \mathrm{~s}$ are 10-times lower than predicted by Eq. 3 . Thus, for low pressures the drying rate is so fast that it is no longer controlled by vapor phase diffusivity alone but is strongly influenced by the interfacial resistance to mass transfer; that is, by the rate at which molecules can escape the liquid into the gas. This effect is also known as a kinetic limitation to evaporative mass transfer as opposed to a purely vapor-phase diffusive limitation. The interfacial mass transfer rate can be predicted using the statistical rate theory (SRT), which accounts for thermallyactivated transitioning of a molecule from a liquid to a vapor phase. The equations of SRT are relatively complex, ${ }^{32}$ but according to Ward and coworkers, they predict accurately the evaporation flux under conditions of low-gas pressure.

When there is a large interfacial barrier to mass transfer (compared to the diffusive barrier in the gas), there is also an interfacial barrier to heat transfer. The temperature on the liquid side of the interface, therefore, falls below that on the vapor side, by $1-5^{\circ} \mathrm{C}$ for water at low temperature, ${ }^{31}$ depending on the pressure and temperature. By heating the vapor phase up above $100^{\circ} \mathrm{C}$, which drives the evaporative flux up to $2 \times 10^{-4} \mathrm{~g} / \mathrm{cm}^{2} \mathrm{~s}$, interfacial temperature discontinuities of up to $30^{\circ} \mathrm{C}$ have been obtained..$^{31}$ Most of the work of Ward and coworkers were carried out for liquids exposed to vapor in a funnel, with the liquid forming a hemispherical interface in the diverging section of the funnel. However, drying of a sessile water droplet was also examined at low pressure (ca. $700 \mathrm{~Pa}$ ) by Ghasemi and Ward, ${ }^{33}$ who found temperature differences of around $1^{\circ} \mathrm{C}$ between the liquid side and the gas side of the interface and, again, rates of evaporation that were limited by interfacial resistance. An interfacial temperature jump is to be expected at low pressures, since the more energetic molecules are the ones that escape the liquid, and the vapor density is too low to allow complete equilibration of temperature across the interface.

A simpler approach than the statistical rate theory for obtaining the evaporation flux in the presence of interfacial resistance to mass transfer is given by the Hertz-Knudsen relationship, as discussed in Ajaev ${ }^{19}$ and Maki and Kumar. ${ }^{20}$ Using this theory, an estimate of the deviation of the liquid temperature at the interface $T_{I}$ from the saturation vapor temperature $T_{\text {sat }}$ (i.e., the interfacial liquid temperature at which the actual interfacial vapor density would equal the saturation value) is given as 20,34

$$
\frac{T_{I}-T_{\mathrm{sat}}}{T_{s}}=J \frac{\sqrt{2 \pi R_{\mathrm{gas}} T_{\mathrm{sat}} / M}}{\alpha \rho_{\mathrm{sat}} \Delta H_{\mathrm{vap}}}
$$

where $J$ is the evaporation rate (mass per unit area per unit time), $M$ is the molar mass of the evaporating liquid, $R_{\text {gas }}$ the gas constant, $\rho_{\text {sat }}$ the saturation vapor density (mass/volume), $\Delta H_{\text {vap }}$ the latent heat of evaporation per unit mass, and $\alpha$ the dimensionless "accommodation coefficient", which is less than unity. The latent heat $\Delta H_{\text {vap }}$ enters the aforementioned equation through the Clausius-Clapeyron equation, which relates equilibrium vapor pressure to $T_{\text {sat }}$ and $\Delta H_{\text {vap. }}$. If $\Delta H_{\text {vap }}$ is large, the equilibrium vapor pressure is sensitive to temperature, and, hence, a drop in liquid interface temperature chokes off evaporation, and thereby limits the evaporative cooling of the interface. Thus, $\Delta H_{\text {vap }}$ acts as a thermostat on the temperature difference $T_{\mathrm{I}}-T_{\text {sat }}$. If $\Delta H_{\mathrm{vap}}$ is large, the right side of the aforementioned equation is small, and the temperature at the interface will approach the saturation value. The numerator $v_{\text {mol }} \approx \sqrt{2 \pi R_{\text {gas }} T_{\text {sat }} / M}, \approx 20 \mathrm{~m} / \mathrm{s}$, is the characteristic velocity of molecules in the gas, and $v_{\text {evap }}=J / \rho_{\text {sat }}$ is the net average velocity (or flux) of vapor moving away from the interface. Thus, ignoring $\alpha$ for the moment, the right side can be written as a product of ratios: $\left(v_{\text {evap }} / v_{\text {mol }}\right)\left(v_{\text {mol }}{ }^{2} / \Delta H_{\text {vap }}\right)$. The first ratio $\left(v_{\text {evap }} / v_{\text {mol }}\right)$ is the average component of vapor velocity directed away from the interface divided by the molecular speed, and this ratio is closely related to the fraction of vapor molecules escaping the interface without recondensing. The second ratio $v_{\text {mol }}^{2} / \Delta H_{\text {vap }}$ is twice the kinetic energy per unit mass of the vapor molecules divided by the latent heat per unit mass. The first ratio approaches unity as the overall pressure is dropped and the mean free path in the gas becomes large. Converting the units of the heat capacities in Table 1 to per unit mass, gives around $1 \mathrm{KJ} / \mathrm{gm} \approx 10^{6} \mathrm{~m}^{2} / \mathrm{s}^{2}$ as a typical value for $\Delta H_{\mathrm{vap}}$, so that the second ratio is $\mathrm{v}_{\text {mol }}{ }^{2} / \Delta H_{\text {vap }} \approx 4 \times 10^{-4}$, which, although small, can be large enough to create a temperature difference of a few degrees, if $v_{\text {evap }} / v_{\text {mol }}$ is near unity, and $\alpha$ is significantly less than unity. If we start by assuming diffusionlimited evaporation, then, from Eq. 3, $v_{\text {evap }}=J / \rho_{\text {sat }} \approx D_{\text {vap }} / R$, where $R$ is the droplet radius, and $D_{\text {vap }} \approx 0.1 \times 10^{-4} \mathrm{~m}^{2} / \mathrm{s}$ is a typical gas-phase diffusivity at atmospheric pressure. From this, we quickly find that the right side of the aforementioned equation is of order $10^{-10} \mathrm{~m} /(R \alpha)=10^{-7} / \alpha$ for $1-\mathrm{mm}$ radius droplets. Thus, even for quite small $\alpha$, there should be negligible temperature jump across the interface, and the assumption of diffusion-limited evaporation is justified unless the rate of vapor diffusion $D_{\text {vap }}$ is very high or the droplet radius $R$ is very small. As expected from these simple arguments, Semenov and coworkers have shown computationally that for evaporating water droplets near room temperature, kinetic effects (i.e., interfacial limitations to mass transfer) come into play when the droplet radius drops below around $1 \mu \mathrm{m} .^{35}$

Most importantly, the temperature jump at the interface should decrease linearly with the rate of evaporation at lowevaporation rates. Thus, when evaporation is slowed greatly by the blanket of air present at atmospheric pressure, which keeps $D_{\text {vap }}$ relatively low, then both the interfacial temperature jump, and the interfacial resistances to heat and mass transfer, should become negligible. In fact, predictions of rates of evaporation that neglect these resistances, i.e., predictions given by Eqs. 3 and 5 are in agreement with experimental measurements of drying water droplets at room temperature and pressure. ${ }^{3}$ Hereafter, in this review, we will neglect interfacial resistances to heat and mass transfer. Those interested in pursuing this issue further should consult the publications of Ward and coworkers, and Oron et al. ${ }^{34}$

We also avoid consideration of vapor recoil, which is the momentum kicked back to the remaining liquid when liquid molecules are launched into the vapor phase by evaporation. 
The recoil is just the reaction to the action of evaporation required by Newton's third law. Since this momentum is usually small, and is directed normal to the droplet's surface, where its effects can be resisted by surface tension, it can usually be neglected unless evaporation is violent. For more discussion of this, see Burelback et al. ${ }^{36}$

\section{Dimensionless Groups Controlling Substrate Heat Transfer \\ Surface/droplet heat resistivity and conductivity ratios $-k_{L} h_{S} / k_{S} h_{0}$ and $k_{S} / k_{L}$}

The temperature distribution within the droplet depends both on the conduction of heat within the droplet and the conduction of heat to the droplet from the underlying substrate. If the substrate has a high-thermal conductivity $k_{S}$, relative to that of the droplet $k_{L}$, then the bottom of the liquid droplet, which is in contact with the substrate, will be isothermal. On the other hand, if $k_{S}$ is small, the liquid will be nonisothermal along the base of the droplet. A reduced value of substrate thermal conductivity will lower the overall temperature in the droplet as well as redistribute the temperature gradient. For the case of a finite substrate thickness, where the bottom of the substrate is assumed to be at ambient temperature, a dimensionless group that controls the temperature distribution in the droplet is $S \equiv k_{L} h_{S} / k_{S} h_{0}$, where $h_{S}$ is the thickness of the substrate and $h_{0}$ is the droplet height. The estimated total temperature difference between the top of the droplet and the bottom of the substrate is then $\Delta T_{\text {tot }}=\Delta T+\Delta T_{\text {substrate }}$ where $\Delta T$, given by Eq. 4 , is the temperature drop across the height of the droplet and $\Delta T_{\text {substrate }}$ is the temperature drop across the thickness of the substrate. The overall temperature drop between the top of the droplet and ambient temperature is thus increased by the factor $1+k_{L} h_{S} / k_{S} h_{0}$ due to resistance to conduction in the substrate. Thus, in this case, the temperature decrease due to drying can be estimated to be

$$
\Delta T_{\text {tot }} \approx \frac{2 \Delta H_{\mathrm{vap}} D_{\mathrm{vap}} \rho_{\mathrm{vap}}}{k_{L}} \frac{h_{0}}{R}\left(1+\frac{k_{L} h_{s}}{k_{S} h_{0}}\right)
$$

Note that when $k_{L} h_{S} / k_{S} h_{0} \gg 1$, the previous expression gives

$$
\Delta T_{\mathrm{tot}} \approx \frac{2 \Delta H_{\mathrm{vap}} D_{\mathrm{vap}} \rho_{\mathrm{vap}}}{k_{S}} \frac{h_{s}}{R}
$$

That is, when the thermal resistance in the substrate is greater than that in the droplet, it dominates the droplet temperature. In such a situation, the lowest droplet temperature is at the contact line, where evaporation is fastest, rather than at the top of the droplet, where the droplet height is greatest, but the evaporation rate is the lowest. An analysis by $\mathrm{Xu}$ et al. ${ }^{37}$ which neglects heat convection, gives the following condition for lowest temperature to be at the contact line: $k_{L} h_{S} / k_{S} R>[\sin 2 \theta-4 \lambda \tan (\theta / 2)] /\left(4 \lambda+2 \sin ^{2} \theta\right)$, where $\theta$ is the contact angle and $\lambda=1 / 2-\theta / \pi$. For small contact angles, less than $30^{\circ}$, this condition is well approximated by $S \equiv k_{L} h_{S} / k_{S} h_{0}>1$. For large contact angles, greater than $45^{\circ}$, increasing the contact angle further decreases the value of the dimensionless group $k_{L} h_{S} / k_{S} h_{0}$ needed to drive the coldest temperature to the contact line, and thereby reverse the Marangoni flow. These theoretical results are supported by experimental observations. 37

If both the droplet height and the substrate are very thin compared to the droplet radius, that is, $h_{S} / R \ll 1$, but $S \equiv k_{L}$
$h_{S} / k_{S} h_{0} \gg 1$, so that the thin substrate nevertheless dominates the resistance to heat transfer, then the evaporative flux along the droplet surface becomes nearly uniform. ${ }^{38}$ In this case, the rate of evaporation becomes proportional to the surface area of the droplet, not to the radius of the droplet, as is the case when the resistance to heat transfer is primarily within the droplet. In the limit of a very flat droplet $(\theta->0)$, instead of Eq. 1, the drying rate in this case (thin substrate, substrate-limited heat transfer) is given by Dunn et $\mathrm{al}^{38}$

$$
-\dot{m}=\frac{\pi k_{s} R^{2}(1-H) \rho_{\mathrm{vap}}}{\Delta H_{\text {sat }} h_{s}\left(d \rho_{\mathrm{vap}} / d T\right)}
$$

Thus, for droplets for which $S \equiv k_{L} h_{S} / k_{S} h_{0} \ll 1$, the longer heat path through the liquid to the top of the droplet will cause the temperature to be lower there rather than at the contact line. ${ }^{12,23}$ In this case, as discussed below, Marangoni flow will cause fluid to be drawn along the free surface toward the top of the droplet, where the temperature is lowest and surface tension highest. For $S \equiv k_{L} h_{S} / k_{S} h_{0} \gg 1$, the lower temperature will be at the contact line, which will reverse this direction of Marangoni flow. Thus, as the droplet dries out, and the droplet height decreases, the value of $S$ passes through unity and the Marangoni flow should switch from inward along the droplet free surface (i.e., "normal" Marangoni flow) to outward along the free surface. According to simulations of $\mathrm{Hu}$ and Larson, ${ }^{12}$ this reversal in direction for $1-\mathrm{mm}$ radius water droplets on a $0.15 \mathrm{~mm}$ thick cover glass substrate should occur at a contact angle of around $14^{\circ}$.

These calculations assume that a fixed temperature is imposed along the bottom of the substrate. Often the temperature assumed for the bottom of the substrate is that of the ambient air temperature far from the droplet. This is reasonable only if the substrate is supported by a solid with highthermal conductivity, such as a metal. Since such conditions are typically only imperfectly realized, most calculations of thermal effects in drying droplets can only be regarded as approximate, at best, even when carried out numerically. The thermal conductivity of glass around room temperature is $k_{g} \approx 2.3 \times 10^{-3} \mathrm{cal} /(\mathrm{cm} \mathrm{sK})=0.96 \mathrm{~W} / \mathrm{mK},{ }^{39}$ which is about $50 \%$ higher than that of water and six times higher than that of simple organic alcohols considered in Table 1. Evaporation of a droplet on a substrate (cork) with lowthermal conductivity of $0.05 \mathrm{~W} / \mathrm{mK}$ was carried out by Safiane and coworkers. ${ }^{38}$

Temperature Profile for Thin Substrates. Numerical solution of the temperature field can be avoided by recourse to a lubrication-type analysis of the temperature field in the substrate and in the droplet, which neglects heat transfer in the radial direction. This is reasonable for relatively flat droplets and substrates that are thin compared to the droplet radius, such as a microscope cover slip, which is often around 0.1 $\mathrm{mm}$ in thickness. The lubrication solution, which also neglects convective heat transfer, and so is valid only for small values of PrRe, is ${ }^{40}$

$$
T_{\infty}-T=\frac{h_{o} \Delta H_{\mathrm{vap}}}{k_{L}} J(r, \theta) \sqrt{1+\left(\frac{\partial h}{\partial r}\right)^{2}}\left(\frac{h(r)}{h_{0}}+\frac{k_{L} h_{S}}{k_{S} h_{0}}\right)
$$

where $T_{\infty}$ is the ambient temperature, and the other parameters have meanings defined earlier. Note that the height profile $h(r)$ is given in Eq. 6. Note also that for small values of 

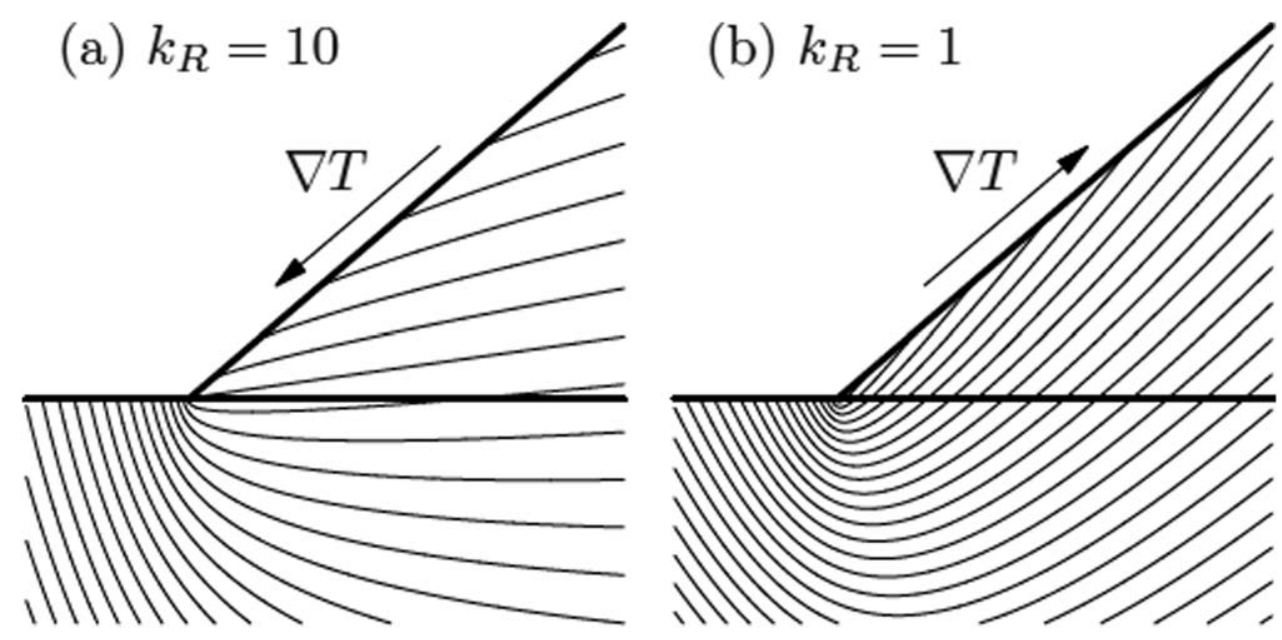

Figure 11. Temperature distributions near the contact line of an evaporating drop with contact angle $\theta=\pi / 8$ (not to scale).

The arrows show the direction of increasing temperature for (a) $k_{R}=k_{S} / k_{L}=10$, and (b) $k_{R}=k_{S} / k_{L}=1$. In the latter case, the slow heat transfer through the substrate, combined with higher evaporation rate at the contact line, leads to a lower temperature at the contact line. (from Ristenpart, Kim, Domingues, Wan, and Stone, ${ }^{23}$ Phys Rev Lett; 99:234502, 2007. Copyright 2007 by the American Physical Society).

$k_{L} h_{S} / k_{S} h_{0}$, the temperature at the contact line where $h(r)=0$, will be equal to the ambient value, which will also be the temperature along the bottom surface of the droplet. Since this expression is only accurate for $\partial h / \partial r \ll 1$, we can simplify the expression further without much loss of accuracy to

$$
T_{\infty}-T=\frac{h_{o} \Delta H_{\text {vap }}}{k_{L}} J(r, \theta)\left(\frac{h(r)}{h_{0}}+\frac{k_{L} h_{S}}{k_{S} h_{0}}\right)
$$

The temperature inside the droplet $\left(z<h_{0}\right)$ is given simply by

$$
\begin{gathered}
T_{\infty}-T(z)=\frac{h_{o} \Delta H_{\mathrm{vap}}}{k_{L}} J(r, \theta)\left(\frac{z}{h_{0}}+\frac{k_{L} h_{S}}{k_{S} h_{0}}\right) \\
\approx \frac{z \Delta H_{\mathrm{vap}}}{k_{L}} J(r, \theta) \text { for small } \frac{k_{L} h_{S}}{k_{S} h_{0}}
\end{gathered}
$$

Thus, the vertical temperature profile is linear within the lubrication approximation. Note, again, that this only holds if thermal convection is small; i.e., for small PrRe. Because of Marangoni convection, which boosts the velocity $U$ by a factor of $\mathrm{Ma}_{\mathrm{U}}$ relative to the mass loss velocity $U_{M L}=R / t_{f}$, thermal convection is generally important unless drying is very slow, i.e., for liquids with volatility similar to hexanol at room temperature, for which $1 \mathrm{~mm}$ radius droplets take more than an hour to evaporate.

Temperature Profile for Thick Substrates. If the substrate is thick compared to the droplet height, or if the substrate is isolated from heat sources, then most or all of the heat that enters the droplet must be lost from the substrate. In that case, both the droplet and the substrate continue to cool until the droplet has completely dried out. This assumes that no heat is gained from the gas phase. (The thermal conductivity of the gas phase $k_{G}$, is typically about an order of magnitude lower than that of most liquids and hence the air is taken to be thermally insulating in most analyses. However, if the gas-phase Grashof number $\mathrm{Gr}_{G}$ is high, heat will be returned to the droplet from the gas phase by heat convection, which will also accelerate evaporation.) Absent heat transport from the gas phase, the droplet temperature will not reach a quasi- steady state, except near the contact line. At quasi-steady state, the radial gradient of temperature at the contact line can be obtained from ${ }^{23}$

$$
\left(\frac{\partial T}{\partial r}\right)_{c}=C r^{-\lambda}\left(\frac{1+\left(k_{S} / k_{L}\right) \tan (\lambda \pi) \tan (\lambda \theta)}{\tan (\lambda \theta)-\left(k_{S} / k_{L}\right) \tan (\lambda \pi)}\right)
$$

where $\lambda(\theta)=0.5-\theta / \pi, \quad C \equiv \Delta H_{\text {vap }} J_{0}(\theta) / k_{L}(2 \cos \theta)^{-\lambda}$, and $J_{0}(\theta)$ is given by Eq. 8. In this case, the radial temperature gradient along the interface can be either positive or negative, depending on the contact angle and the ratio of conductivities of the fluid and the substrate, with a critical value of this ratio given by ${ }^{23}$

$$
\left(\frac{k_{S}}{k_{L}}\right)_{c}=\tan (\theta) \cot \left(\frac{\theta}{2}+\frac{\theta^{2}}{\pi}\right)
$$

For values of $k_{S} / k_{L}$ above the critical value given previously, the temperature decreases from the contact line toward the center of the droplet, while the reverse occurs for values below this critical value; see Figure 11. Notice that the critical condition does not in this case involve the thickness of the substrate or height of the droplet, since the analysis was carried out only for the temperature gradients near the contact line, and the substrate is taken to be infinitely thick. The analysis predicts that for $k_{S} / k_{L}$ less than around 1.5, the flow along the free surface is toward the contact line; i.e., it is a reverse Marangoni flow. This occurs because the drying rate is fastest at the contact line and if the substrate conductivity is not high enough, there will be a steep dip in substrate temperature, and, hence, a highersurface tension, there. If the substrate thermal conductivity is high, however, the substrate stays warmer than the overlying droplet, even close to the contact line, and so the temperature close to the contact line, where the fluid is thin, will be higher than elsewhere, despite the high rate of evaporation there.

The analysis of Ristenpart et al. ${ }^{23}$ breaks down outside of the vicinity of the contact line, and for finite substrate thickness, the analysis of $\mathrm{Xu}$ et al. ${ }^{37}$ discussed earlier, should be more valid. 
Finally, it has been observed that as one moves along the liquid-air interface, there can be a reversal in flow direction in a drying water droplet. This reversal occurs at a stagnation point that is within around $10 \mu \mathrm{m}$ of the contact line, and is thought to arise from the presence of a thin wetting film that begins at the nominal macroscopic contact line and extends along the substrate away from the droplet. Heat transfer effects in this film and the attached droplet are thought to produce the local reversal in flow direction. ${ }^{41}$

\section{Heated substrate radius ratio and evaporation number}

$-R_{s} / R$ and $E \equiv \frac{1}{\operatorname{RePr}}\left(\frac{c_{p} \Delta T}{\Delta H_{\text {vap }}}\right)$

Given the sensitivity of the droplet flow field to temperature and to thickness and thermal conductivity of the substrate, it is no surprise that heating a portion of the substrate, even modestly, can have a large effect on the flow within the drying droplet. For example, if one heats a circular patch of radius $R_{s}$ centered on the droplet center, the flow pattern is controlled by the ratio $R_{S} / R$ of the radius of the heated patch to the radius $\mathrm{R}$ droplet. When $R_{s} / R>1$, the contact line is warmer than the apex of the droplet, and normal Marangoni flow results, with liquid pulled to the apex from the contact line. When $R_{S} / R<1$, the heat applied near the droplet center is transported to the apex and the Marangoni flow reverses direction. These results were demonstrated through numerical solution by Girard and Antoni. ${ }^{42}$ It is of interest that the rate of drying of a small $2 \mu \mathrm{L}$ water droplet substrate heated to as high as $50^{\circ} \mathrm{C}$ is not significantly affected by the heating. ${ }^{43}$

In recent work by Sefiane and coworkers, ${ }^{18}$ a uniformly heated substrate led to convective rolls and "hydrothermal waves" in a sessile drying water droplet, whose temperature field, imaged by infrared thermography, showed complex thermal patterns. A computational analysis showed the formation of Marangoni rolls, the number of which depends on the Prandtl number. Because the substrate is heated, the evaporation number $E$ for this problem is an independent control parameter, whose value is small, around $10^{-5}$ for the conditions of these experiments. The small value of $E$ results from the large ratio of $\Delta H_{\mathrm{vap}} / c_{p, L} \approx 500^{\circ} \mathrm{C}$ for typical liquids. The small value of $E$ means that the rate of volume loss produced by evaporation is relatively small compared to the rate at which liquid can be delivered by flow to the region of drying for most of the droplet. However, near the droplet contact line where the liquid layer is thin, the flow is choked off, so that evaporated liquid is not readily replaced by flow, and the liquid may dry out, leading to movement of the contact line. The moving contact line can then disturb the flow throughout the droplet. This effect is clearly seen in the simulations of Karapetsas et al. ${ }^{18}$ whose results are sensitive to the values of $E$, despite their small magnitudes. As will be discussed later, near the contact line, the physics of thin films may become important, and to describe this, an evaporation number that includes these thin-film physics has been introduced by Frastia et al. ${ }^{44}$

Vapor pressure sensitivity ratio -

$\beta_{\text {vap }} \Delta T \approx \beta_{\text {vap }} \frac{\Delta H_{\text {vap }} D_{\text {vap }} \rho_{\text {vap }}}{k_{L}} \frac{h_{0}}{R}=\frac{\beta_{\text {vap }} \Delta T}{E \cdot S r}\left(\frac{D_{\text {vap }} t_{f}}{R h_{0}}\right) \frac{\rho_{\text {vap }}}{\rho_{L}} \frac{h_{0}}{R}$

If the temperature at the droplet free surface is reduced, this will proportionately reduce the vapor pressure (or vapor concentration) of the liquid and so slow the droplet drying. The change in vapor mass density, if modest, can be estimated as $\beta_{\text {vap }} \rho_{\text {vap }} \Delta T$, where $\Delta T$ is the temperature drop due to latent

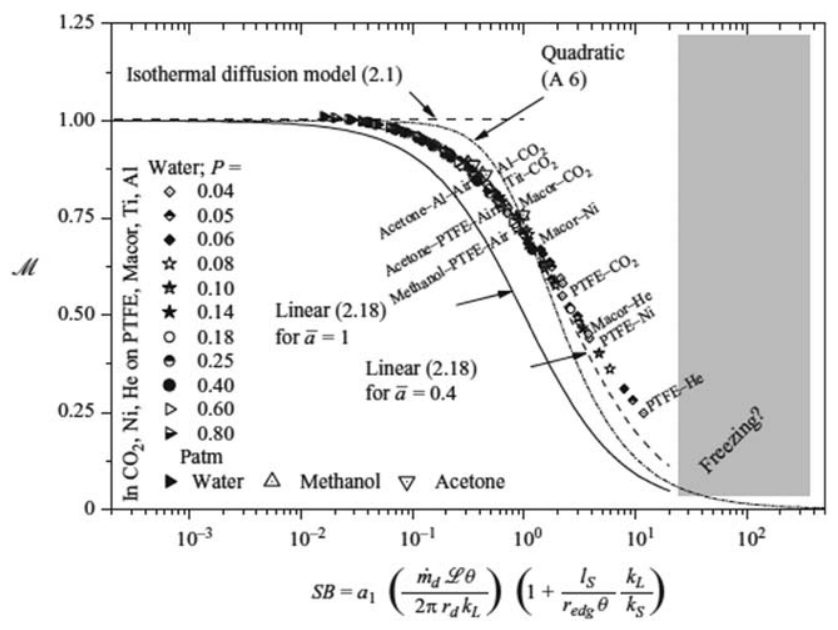

Figure 12. Validation of Eq. 23 against experiments, where the ratio of evaporation rate to its rate in the absence of droplet cooling $M$, is plotted as a function of the dimensionless number SB.

(Here $r_{d}$ is the droplet radius, designated $R$ elsewhere in this review, and $\dot{m}_{d}=\pi r_{d} D_{\text {vap }} \rho_{\text {vap }}, L=\Delta H$ is the latent heat of evaporation, and $\theta \approx h_{0} / 2 R$ is the contact angle. Also $\ell_{s}=h_{s}$ is the substrate thickness, $r_{\text {edg }} \theta=h_{\text {edg }}$ is the height of the droplet near the droplet edge, and $a_{1}=\beta_{\text {vap. }}$. Thus, the definition of SB in the figure above matches that used in Eq. 22 (from Sefiane and Bennacer, ${ }^{45} J$ Fluid Mech; 667:260-271, 2011, with permission from Cambridge University Press).

heat of evaporation and $\beta_{\text {vap }} \equiv d \ln \left[\rho_{\text {vap }}(T)\right] / d T$ is the temperature coefficient of the vapor pressure. Using $\Delta T$ from Eq. 4 , we obtain a relative reduction in vapor concentration of $\Delta \rho_{\text {vap }} / \rho_{\text {vap }} \approx \beta_{\text {vap }} \Delta T \approx \beta_{\text {vap }} \frac{\Delta H_{\text {vap }} D_{\text {vap }} \rho_{\text {vap }}}{k_{L}} \frac{h_{0}}{R}$. The reduction in relative evaporation rate (i.e., change in rate normalized by the unchanged rate) will be of similar magnitude to $\Delta \rho_{\text {vap }} / \rho_{\text {vap. }}$. Thus, if this dimensionless group is 0.5 , the evaporation rate will be reduced by about a factor of two or so, with a corresponding reduction in fluid velocity within the droplet. Very volatile liquids, such as acetone, or water at low-gas pressure (so that vapor diffusion is rapid), can have large values of $\beta_{\mathrm{vap}} \Delta T$, and, hence, large reductions in rates of evaporation relative to their rates in the absence of droplet cooling. If the substrate thermal resistance impedes heat transport, then this reduction in drying rate will be augmented by an additional factor of $1+k_{L} h_{S} / k_{S} h_{0}$. The liquids in Table 1 , at room temperature, have quite small values of $\beta_{\text {vap }} \Delta T$, less than around 0.1 , or so, so the evaporation rate will not be changed much by evaporative cooling of the droplet. Thus, in those cases the evaporation rate can be estimated using the droplet vapor pressure at ambient temperature.

The case in which the droplet surface cools enough to reduce its temperature sufficiently to affect the rate of evaporation was studied experimentally and theoretically by Sefiane and Bennacer, ${ }^{45}$ who were able to correlate results for various droplet fluids and substrates of various thermal conductivities using the parameter

$$
S B \equiv \beta_{\text {vap }}\left(\frac{\Delta H_{\mathrm{vap}} D_{\mathrm{vap}} \rho_{\mathrm{vap}}}{k_{L}} \frac{h_{0}}{R}\right)\left(1+\frac{k_{L} h_{S}}{k_{S} h_{e d g}}\right)
$$

Here the product of the first two terms is just the vapor pressure sensitive ratio $\beta_{\text {vap }} \frac{\Delta H_{\text {vap }} D_{\text {vap }} \rho_{\text {vap }}}{k_{L}} \frac{h_{0}}{R}=\frac{\beta_{\text {vap }} \Delta T}{E \cdot S r}\left(\frac{D_{\text {vap }} t_{f}}{R h_{0}}\right)$ 
$\frac{\rho_{\text {vap }}}{\rho_{L}} \frac{h_{0}}{R}$, where the second expression is written in terms of the dimensionless parameters defined in the previous section on dimensionless groups. The vapor density $\rho_{\text {vap }}$ in this equa-
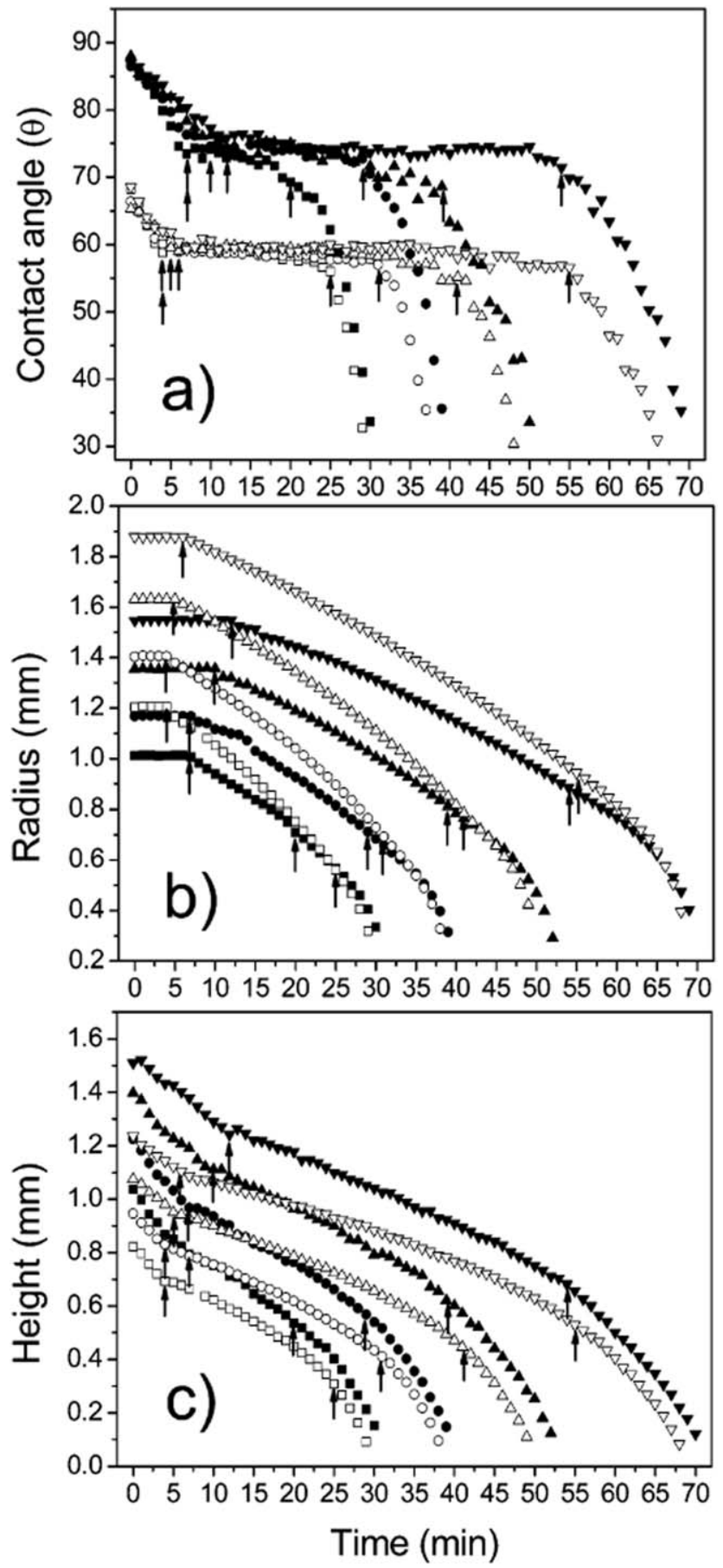

Figure 13. Shape changes of water droplets as a function of evaporation time on PMMA surfaces (open symbols) and PAMS surfaces (closed symbols) with initial masses of around $2 \mathrm{mg}$ (squares), $3 \mathrm{mg}$ (circles), $5 \mathrm{mg}$ (triangles pointing up), and $7.5 \mathrm{mg}$ (triangles pointing down): (a) contact angle, (b) contact radius, and (c) droplet height.

The arrows mark the times at which transitions in drying regimes occur, as discussed in the text (Reprinted with permission from Kim, Ahn, Kim, and Zin, ${ }^{46}$ Langmuir; 23:61636169, 2007. Copyright 2007 American Chemical Society). tion is taken to be the equilibrium vapor density at ambient temperature, not the actual vapor density at the temperature of the droplet surface, which can be reduced by evaporative cooling. The remaining term $\left(1+\frac{k_{L} h_{S}}{k_{S} h_{\mathrm{edg}}}\right)$, is similar to the corresponding term in Eq. 14, except that in Eq. 22, the droplet height $\left(h_{0}\right)$ has been replaced by the droplet height somewhere near the droplet edge $\left(h_{\text {edg }}\right)$, where evaporation is rapid. Sefiane and Bennacer ${ }^{45}$ plotted $\mathrm{M} \equiv \dot{m} / \dot{m}_{0}$, which is the ratio of the droplet drying rate $-\dot{m}$, normalized by the drying rate in the absence of evaporative cooling. This latter quantity is $-\dot{m}_{0}=\pi R D_{\text {vap }}(1-H) \rho_{\text {vap }}\left(0.27 \theta^{2}+1.30\right)$ according to Eq. 3. Their plot is shown in Figure 12, and it includes drying rates for water, ethanol, and acetone, drying over a substrates aluminum, titanium, Macor (a type of ceramic) and Teflon, with atmospheres of helium, carbon dioxide and nitrogen, at pressures ranging from 0.04 to 1 atm. This range of conditions provided a broad enough range of values of SB for the effect of evaporative cooling on drying rate to be thoroughly explored. Notice in Figure 12 that for $\mathrm{M}$ below around 0.1 , there is little effect of evaporative cooling on drying rate, but for higher values of $M$, the drying rate drops much lower. Two of the lines plotted in Figure 12 correspond to the simple equation

$$
M=\frac{1}{1+\bar{a} S B}
$$

with the dimensionless constant $\bar{a}$ assigned the value 1 or 0.4 . Results for a model in which SB appears squared (the "quadratic" model) are also plotted; see Sefiane and Bennacer $^{45}$ for details. These simple equations provide useful methods of estimating when evaporative cooling will influence droplet drying times and how large this influence will be.

\section{Moving Contact Line and Precursor Films}

So far, we have focused on the case of a pinned contact line, which fixes the droplet radius during evaporation.

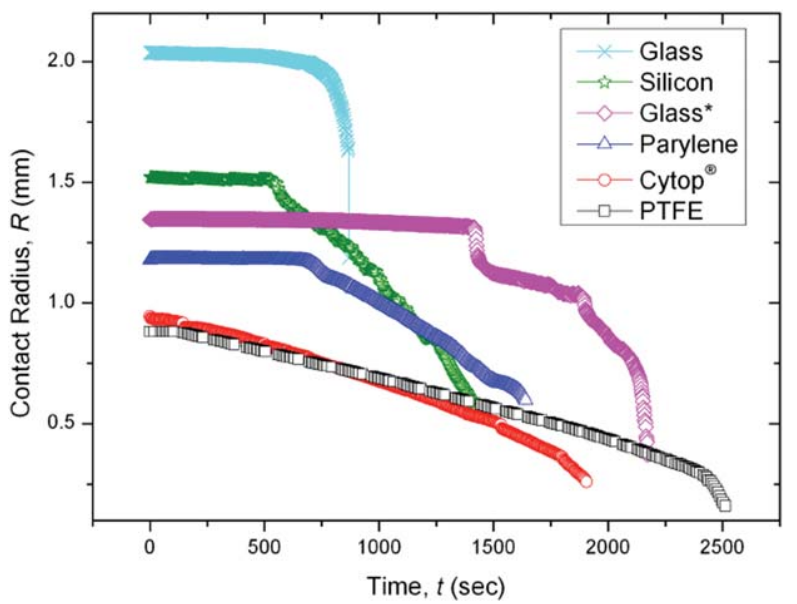

Figure 14. Evolution of the contact radius $\mathbf{R}$ on: glass (crosses(x)), silicon (stars), glass* (diamonds), Parylene (triangles), Cytop ${ }^{\circledR}$ (circles) and PTFE (squares) plotted vs. time, $t$ (in seconds), for water (Reprinted with permission from Orejon, Sefiane, and Shanahan, ${ }^{47}$ Langmuir; 27:12834-12843, 2011; Copyright 2011 American Chemical Society). 
While this assumption holds for some droplets throughout most of the evaporation process, in other cases, the contact line recedes soon after drying starts. In essentially all cases, the contact line eventually recedes, although perhaps only during the last seconds of drying.

Roughly speaking, there are three modes of contact line motion: (1) pinned contact line, decreasing contact angle, (2) constant contact angle, decreasing droplet radius, and (3) decreasing of both radius and contact angle. All three modes can be seen in Figure 13, for drying water droplets on poly (methyl methacrylate) (PMMA) and poly ( $\alpha$-methyl styrene) (PAMS) polymer surfaces. For these droplets and substrates, the initial contact angle is not too far from $90^{\circ}$, and each mode of drying persists for a significant period of time, depending on the initial droplet volume. Initially, the droplet contact line is pinned (Figure 13b), and the contact angle is forced to decrease with time (Figure 13a). After the contact angle has decreased significantly, the contact line begins to recede, with the contact angle fixed at a value lower than the initial value. Eventually, the contact angle begins to decrease again, while the contact line continues to move inward.

Some examples of the change in water droplet radius with time of drying for a wider range of substrate wettabilities are shown in Figure $14 .^{47}$ Note in Figure 14 that for a low-initial contact angle, and large-initial radius, namely water on glass, (top line on Fig. 14) the first drying regime dominates as the droplet radius remains nearly constant until almost the completion of drying, and then it rather abruptly collapses. For the hydrophobic surfaces coated with Cytop ${ }^{\circledR}$ (circles) and PTFE (squares), the contact angle is initially greater than $90^{\circ}$, and there is essentially no regime of pinned contact line; the contact angle remains roughly constant for a time as the contact line retreats (Figure 14-bottom lines). Intermediate cases, with initial contact angles between 60 and $90^{\circ}$, show behaviors similar to that displayed in Figure 13 -the contact line is initially pinned, but depinning occurs well before the droplet has completely dried. In general, pinning is more prevalent when the initial contact angle is less than $90^{\circ}$, but for contact angles greater than $90^{\circ}$, the pinning regime can be absent. However, there are exceptions to this: Shin et al. ${ }^{48}$ observed pinning of a water droplet on a substrate coated with octadecyltrichlorosilane (OTS), with a contact angle of $120^{\circ}$. Pinning on this hydrophobic surface was evidently caused by surface roughness. Another exception, in the opposite direction, is water on atomically smooth mica, which despite having a contact angle less than $90^{\circ}$, shows essentially no pinning. ${ }^{1}$

Thus, the pinning or movement of the contact line is governed by both wetting force and surface roughness. For an ideally flat, chemically homogeneous, surface, the contact angle should theoretically always remain at its equilibrium value $\theta_{0}$, given by the Young equation

$$
\gamma_{S G}-\gamma_{S L}=\gamma \cos \theta_{0}
$$

Here $\gamma$ is the liquid/air surface tension, while $\gamma_{S G}$ and $\gamma_{S L}$ are the substrate/gas and the substrate/liquid surface tension (or surface energy per unit area). When the liquid is first deposited onto a relatively smooth homogeneous surface, the initial contact angle, produced by spreading of the liquid onto the surface, is called the advancing angle $\theta_{A}$, and it can be larger than the equilibrium angle $\theta_{0}$. When the droplet evaporates, the contact line has no thermodynamic driving force to retreat until the contact angle has dropped below $\theta_{0}$. Thereafter, although there is a driving force to retreat, the contact line can remain pinned until the contact angle has

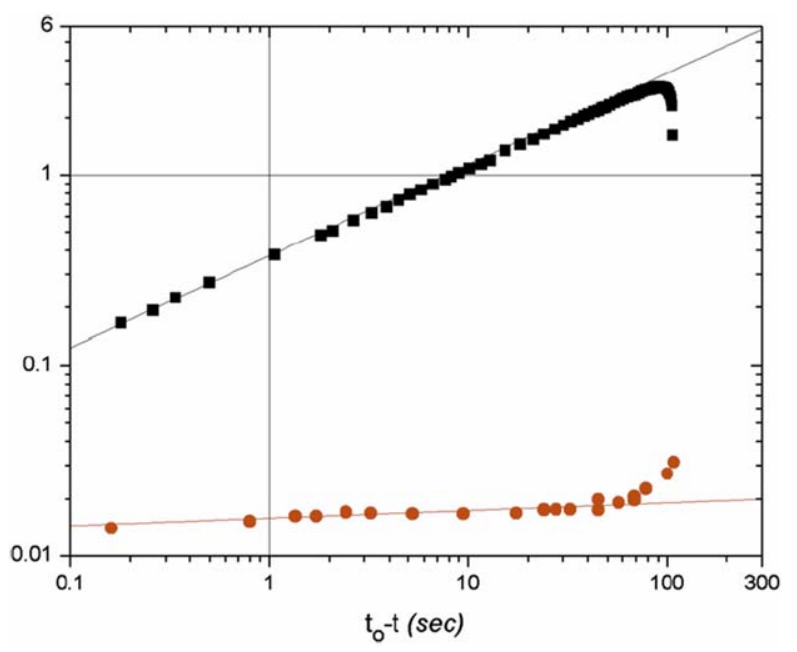

Figure 15. Droplet radius, upper curve, left axis in $\mathbf{m m}$, and apparent contact angle, lower curve, right axis in radians, for an octane droplet, volume $1 \mu \mathrm{l}$, deposited on a silica wafer, under complete wetting conditions as a function of time remaining until complete evaporation $t_{0}-t$, where $t_{0}$ is the drying time, designated $t_{f}$ elsewhere in this review.

Immediately after droplet deposition (right), the radius increases and the contact angle decreases rapidly as can be seen starting on the on the far right and moving leftward with increasing drying time. Then the radius reaches a maximum and afterwards decreases according to a power law (straight line, upper curve). During drop retraction, the angle can also be fitted to a power law (straight line, lower curve). Within the last $0.5 \mathrm{~s}$, the angle decreases more rapidly (not shown). The values of the exponents are $y=0.48$ for the upper curve and $x \sim 0.04$ for the lower one (Reprinted from $J$ Coll Interf Sci, 312, Guéna, Poulard, and Cazabat, ${ }^{49}$ The leading edge of evaporating droplets, 164-171, Copyright 2007, with permission from Elsevier). [Color figure can be viewed in the online issue, which is available at wileyonlinelibrary.com.]

decreased to a still lower value, called the retreating angle $\theta_{R}$. In the experiments of Figure 13, the advancing and receding angles $\theta_{A}$ and $\theta_{R}$ were directly measured by ejecting a liquid droplet onto a surface and then withdrawing this liquid using a needle syringe. ${ }^{46}$ The values of $\theta_{A}$ and $\theta_{R}$ measured this way were close to the contact angles measured at the start of drying and at the first transition from pinned to moving contact line, shown in Figure 13a. The difference between $\theta_{A}$ and $\theta_{R}$ is known as the contact angle hysteresis, and it is evidently responsible for the existence of the initial pinned contact line, and the transition to the second regime of a moving contact line with fixed contact angle. For the smooth hydrophobic surfaces considered in the bottom two curves of Figure 14, the hysteresis is evidently small, since the contact line retreats immediately after drying starts, and the contact angle during retreat initially remains close to its initial advancing value. The driving force for contact line retreat can be expressed in terms of the deviation $\delta \theta$ of the contact angle from its equilibrium value. Thus, this driving force $d F$, can be estimated from Eq. 24 to be ${ }^{47}$

$$
d F=\gamma \cos \left(\theta_{0}-\delta \theta\right)-\gamma \cos \left(\theta_{0}\right) \approx \gamma \sin \left(\theta_{0}\right) \delta \theta
$$

Note from Eq. 25 that as $\theta_{0}$ increases from $0^{\circ}$ to around $90^{\circ}$, $\sin \left(\theta_{0}\right)$ increases, and, hence, a smaller $\delta \theta$ is required to 

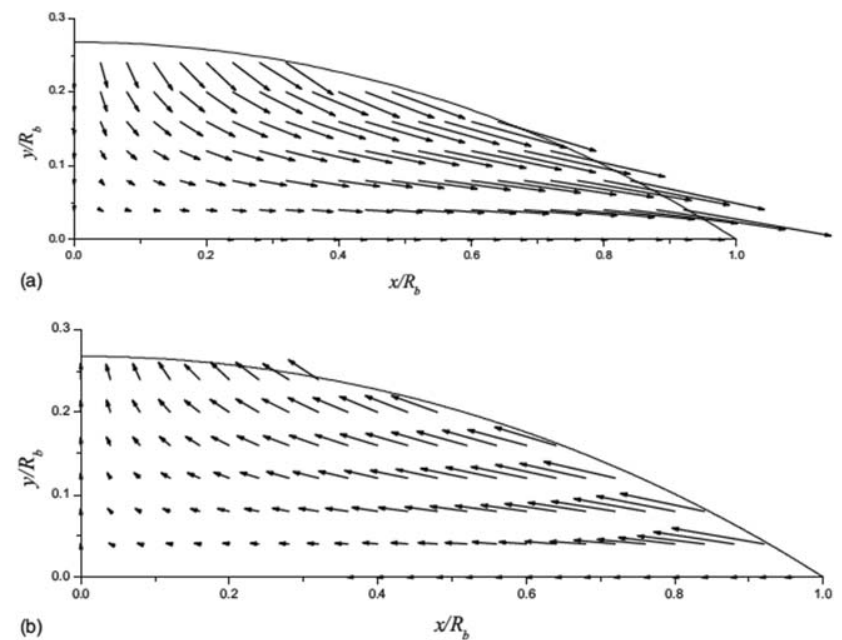

Figure 16. Flow field in an evaporating cylindrical-cap droplet with uniform evaporation flux, with contact angle $\theta=30^{\circ}$.

For (a) a pinned contact line, and (b) a moving contact line with fixed contact angle. In both cases the slip length divided by droplet radius $R_{b}$ was set to $10^{-3}$ (from Petsi and Burganos, ${ }^{5}$ Phys Rev E.; 78:036324, 2008. Copyright 2008 by the American Physical Society).

increase the driving force enough to force the contact line to retreat. This can explain the rapid retreat of the contact line when the contact angle is near $90^{\circ}$, compared to the case when it is small.

A special case is that of nearly complete wetting, where the equilibrium contact angle is close to zero, on smooth flat surfaces. Guéna et al. ${ }^{49}$ have studied this case in detail using very clean flat silica surfaces and volatile liquids. An example of their results is shown in Figure 15, where the droplet radius and contact angle are plotted against the complementary time $t_{f^{-}} t$ (given as $t_{0}-t$ in Figure 15 ), which is the time remaining before complete evaporation. Thus, the values of early radius and contact angle start at the right side of Figure 15 , and the data for later times in the drying process are plotted leftward from there. In the short initial period (on the far right) the spreading of the droplet across the surface shows as an increase in droplet radius with time since the start of drying. This spreading is governed by "Tanner's laws", which for pure liquids give droplet radii that increase as $t^{1 / 10}$ or $t^{1 / 8}$, depending on whether the radius is, respectively, smaller than, or larger than, the capillary length $h_{\text {cap }} \approx \sqrt{\sigma / \rho_{L} g} \cdot{ }^{50,51}$ (The exponent increases to around $1 / 4$ if solute is present so that there is Marangoni flow. ${ }^{52,53}$ ) Eventually, due to evaporation and diminishing contact angle, the droplet contact line stops advancing and immediately retreats without pinning, in the case considered here of a wetting fluid on a smooth surface. This retreat is also described by a simple power law, but written in terms of $t_{f} t$, the time remaining until complete drying. This law is $R \propto\left(t_{f}-t\right)^{y}$, where $y=0.48$ for case plotted in Figure 15. Mass balance dictates that this exponent is related to that for the contact angle $R \propto\left(t_{f}-t\right)^{y}$ by the equation $2 y+x=1$. If the contact angle were to remain at the equilibrium value of zero during contact line motion, then $x=0$, and $y$ would be 0.5 . A small deviation from this is seen for organic liquids on smooth surfaces, but for water, the deviation is much greater, and a value of $y=0.61 \pm 0.03$ is seen for water on mica. ${ }^{54}$ This anomalously large value was traced to the natural convection of water vapor, which is less dense than the surrounding air, leading to accelerated evaporation and greater deviation from an equilibrium contact angle. This explanation was confirmed both by suppressing natural convection for water vapor by using confinement, and inducing convection for the heavier organic liquids using a fan.

The effects of pinned or unpinned contact lines on the flow in the drying droplet have been analyzed numerically by Petsi and Burganos and by Masoud and Felske. ${ }^{14}$ Petsi and Burganos ${ }^{15}$ considered a cylindrical cap droplet while Masoud and Felske took the droplet to be a spherical cap. There were also differences in boundary conditions near the contact line; Petsi and Burganos used a slip boundary condition to alleviate otherwise infinite stresses there, while Masoud and Felske avoided infinite evaporative flux at the contact line by modifying the diffusive flux expression (e.g., Eq. 7) so that the evaporation rate near the contact line dropped to zero. The flows
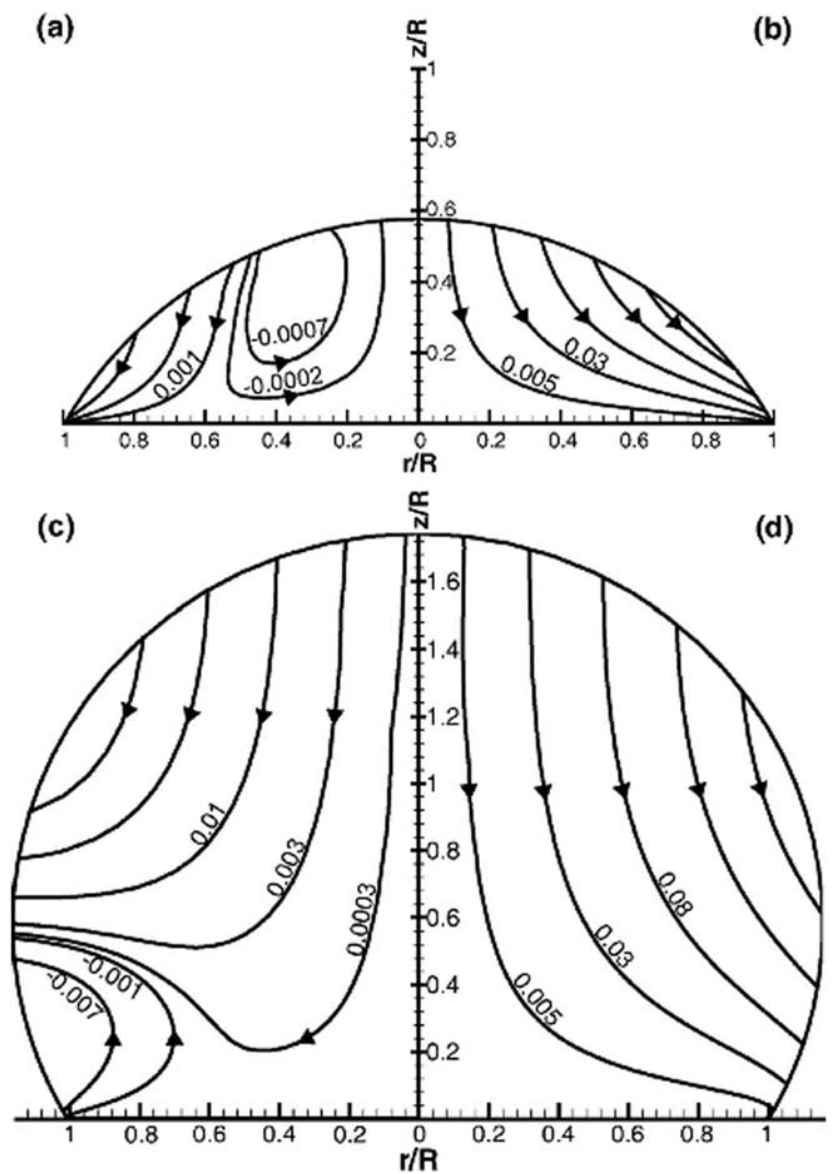

Figure 17. Contours of non-dimensional stream function for viscous flow inside a spherical-cap droplet exposed to non-uniform evaporative flux determined by vapor-phase diffusion, but modified near the contact line so that the flux drops rapidly to zero at the contact line to remove the edge singularity.

(a) $\theta=60^{\circ}$, freely moving contact line (constant contact angle) with shown values of dimensionless stream function, (b) $\theta=60^{\circ}$, pinned contact line, (c) $\theta=120^{\circ}$, freely moving contact line, and (d) $\theta=120^{\circ}$, pinned contact line (Reprinted with permission from Masoud and Felske, ${ }^{14}$ Phys Fluids; 21:042102, 2009. Copyright 2009, AIP Publishing LLC). 

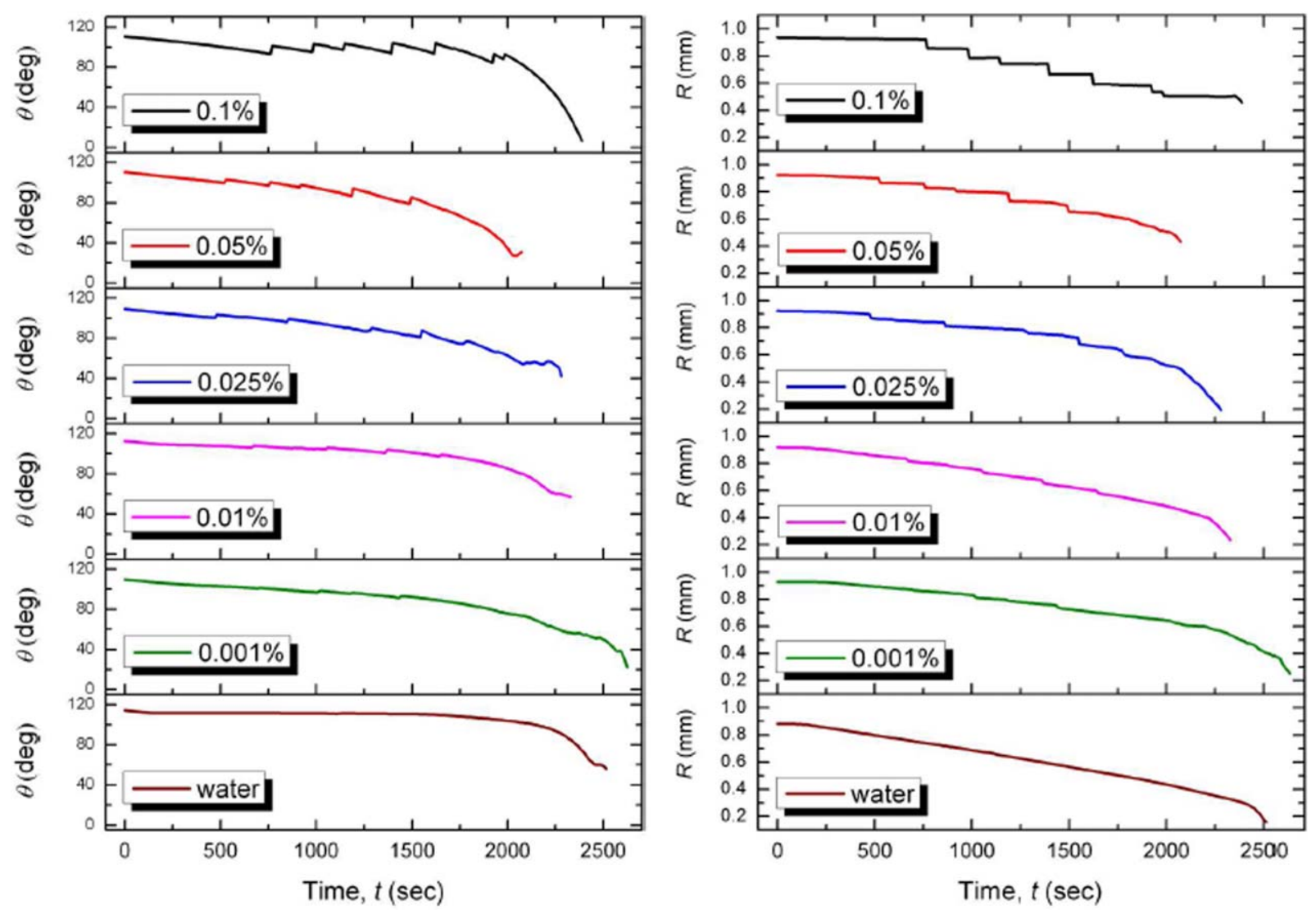

Figure 18. (left) Evolution of the contact angle, $\theta$ (degrees), with time and (right) contact radius $R$, with time, $t$ (in seconds), for different TiO2 concentrations in water on PTFE (Reprinted with permission from Orejon, Sefiane, and Shanahan, ${ }^{47}$ Langmuir; 27:12834-12843, 2011; Copyright 2011 American Chemical Society).

[Color figure can be viewed in the online issue, which is available at wileyonlinelibrary.com.]

resulting from pinned and constant-contact angle conditions are compared in Figures 16 and 17. For the relatively flat (contact angle $\theta=30^{\circ}$ ) cylindrical cap, the change from fixed to moving contact line transformed the flow from outwards (Figure 16a) to inwards (Figure 16b). However, for the spherical droplets with larger contact angles $\left(\theta=60\right.$ and $\left.120^{\circ}\right)$ and somewhat different boundary conditions described previously, the outward-directed flow for the pinned contact line (right- $b$ and $\mathrm{d}$ in Figure 17), is changed to a flow with a surface stagnation point when the contact line is allowed to move (left- a and $\mathrm{c}$ in Figure 17).

Deposition of solute at the contact line is expected to increase the hysteresis in contact angle due to particle-induced pinning. Particle-induced pinning was demonstrated by Dee$\operatorname{gan}^{2}$ on atomically smooth mica, and by Weon and Je, ${ }^{55}$ who observed the spreading and retreat of droplets of decalin on clean glass both without and with 0.1 and $1 \mu \mathrm{m}$ radius PMMA particles. The particle-free droplets could both spread by Tanner's law and then retreat because of evaporation. In contrast, the presence of the suspended particles caused pinning of the contact line, once their concentration there reached a linear density of $10 \%$ along the droplet perimeter, in agreement with a theoretical prediction. ${ }^{55}$ The pinning effect of particles was also observed by Sefiane and coworkers, ${ }^{47}$ when they added $\mathrm{TiO}_{2}$ nanoparticles to water droplets drying on hydrophobic PTFE surfaces; see Figure 18. Notice in Figure 18 that at higher $\mathrm{TiO}_{2}$ concentrations the contact angle and contact line radius change discontinuously in a series of jumps. These jumps represent depinning events, followed by repinning of the contact line. The depinning occurs when the contact angle has dropped enough that wetting forces causing the contact line to retreat bring the contact angle below the equilibrium value. During the retreat, the contact angle increases toward the equilibrium value, and the wetting force driving the retreat weakens, leading eventually to repinning. This is again reinforced by further deposition at the new contact line, and the cycle repeats. The deposition pattern resulting from this will be shown in the next section. Here, we simply remark that the pinning and depinning of a droplet contact line during drying is a complex process, controlled by liquid-solid, liquid-air, and solid-air surface energy and surface roughness, which can be time-dependent, if solid is deposited during evaporation. Cyclic pinning and depinning is even observed when a volatile droplet containing nanoparticles is deposited onto an immiscible viscous liquid, ${ }^{56}$ so that the solid substrate is replaced by a liquid; in this case there is no surface roughness and therefore the contact angle hysteresis is entirely due to particle deposition at the contact line. Pinning in the presence of suspended particles can be influenced by particle size. For example, in the studies of Sangani et al. ${ }^{57}$ smaller particles (ca. $3 \mu \mathrm{m}$ in diameter or smaller) were transported to the contact line, while larger $50 \mu \mathrm{m}$ particles migrated to the droplet center. Pinning and depinning can even be affected by the thermal conductivity of substrate material that lies underneath the material that directly contacts the liquid. ${ }^{47}$ Theories for rates of movement of contact lines must, therefore, contend with these factors, as well as with hydrodynamic and physicochemical effects. 


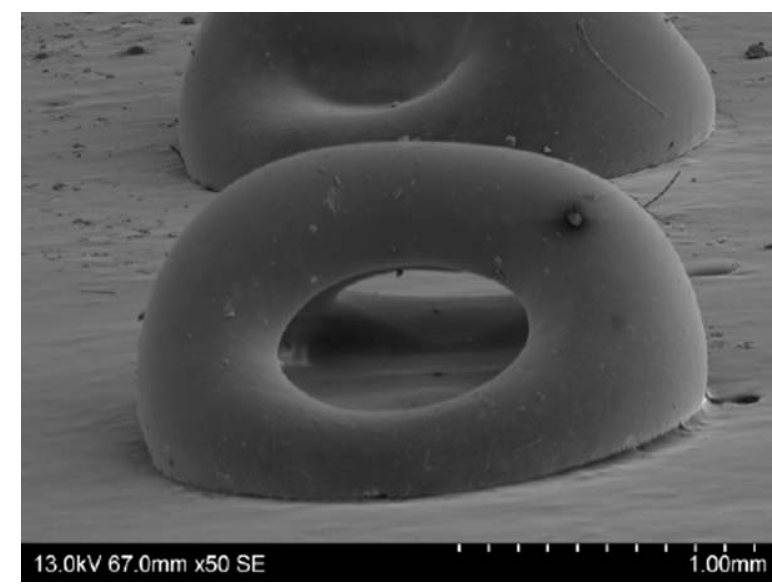

Figure 19. Arched structure formed by alumina colloids deposited during droplet drying.

This pattern was controlled by the droplet spacing, which affected the pattern of vapor density, which in turn patterned the evaporation rate on the droplet surface, leading to the structures shown (Reprinted with permission from Chen and Evans, ${ }^{61}$ Langmuir; 25:11299-11301, 2009. Copyright 2009 American Chemical Society).

There have been a number of efforts to develop theories for contact line motion. While this topic warrants a review of its own, here we briefly remark that wetting effects in liquid films on solid surfaces are often accounted for through the concept of a disjoining pressure, which arises from a thickness-dependent free energy of a liquid film. ${ }^{50}$ Disjoining pressure is a driving force for flow of the liquid in response to the van der Waals and polar interactions between the substrate material and the liquid film. These forces lead to rapid formation an ultra-thin precursor film on the substrate that extends well beyond the macroscopic contact line of the droplet. ${ }^{50,58}$ This film, whose thickness is in the nanometer range, connects continuously to the bulk of the droplet. Hence, when one zooms in to the nanometer scale, the macroscopic contact line disappears and is replaced by a transition region from the bulk droplet to a thin substrate-adsorbed film. Analyses that consider wetting forces, including disjoining pressure, no longer contain an explicit contact line or contact angle as boundary conditions. Equations that include these wetting forces, within a lubrication approach, can be found in Ajaev. ${ }^{19}$ These equations describe both the initial time-dependent spreading of the droplet on the surface and its retreat as the liquid dries out. Such equations have been used, for example, to explain the fingering instability observed on drying of thin films. ${ }^{59}$

Recently, using a lubricated wetting analysis similar to that of Ajaev, efforts have been made to include the effect of suspended particles on droplet flows. ${ }^{44,60}$ These analyses are too complex to be described in detail here, but are beginning to predict deposition patterns that can be compared to those in experiments, as will be discussed in the next section.

\section{Deposition Patterns}

Deposition patterns are in general sensitive not only to the flow pattern in the droplet, but also to the diffusive, rheological, and surface-active properties of the deposited material. Generalizations are difficult, but one important consideration is the diffusivity $D$ of the dispersed material. The value of the dimensionless group $D t_{f} / R^{2}$ will control the degree to which the suspended material can diffuse across the radius $R$ of droplet in the time $t_{\mathrm{f}}$ required for drying. For 1-mm (or $0.1 \mathrm{~cm})$ droplets that dry in around $20 \mathrm{~min}(1000 \mathrm{~s})$, diffusivities $D$ much lower than $10^{-5} \mathrm{~cm}^{2} / \mathrm{s}$ will prevent the solute from dispersing throughout the droplet. Since even small molecules in low-viscosity solvents have diffusivities as low as $10^{-5} \mathrm{~cm}^{2} / \mathrm{s}$, we do not expect much larger polymer molecules or colloids to be able to diffuse across the droplet during drying, but instead will become highly nonuniformly concentrated. During the final stages of drying, even smallmolecule solutes will not be able to diffuse rapidly enough to keep up with nonuniformities in concentration produced by rapid convection and drying effects, and will therefore typically deposit nonuniformly on the substrate.

As discussed in the Introduction, the first deposition pattern to attract significant attention was the "coffee ring" pattern (Figure 1), first explained by Deegan et al., ${ }^{1}$ and supported by later numerical studies by Fischer. ${ }^{13}$ The work of Deegan et al., ${ }^{1}$ and further studies discussed later, have shown that the coffee ring deposition at the contact line only occurs when (1) the evaporation of liquid at or near the contact line is not somehow suppressed, (2) the contact line is pinned at one location for a significant fraction of the drying time, (3) the solute or particles are not significantly deposited onto the substrate before they can be convected outwards to the contact line, (4) the outward flow driven is not counteracted by inward Marangoni flow, (5) the deposit at the contact line is not swept inward during the last stages of droplet evaporation, and (6) the colloid to be deposited does not form a jammed state on the droplet free surface, preventing its being transported to the contact line.

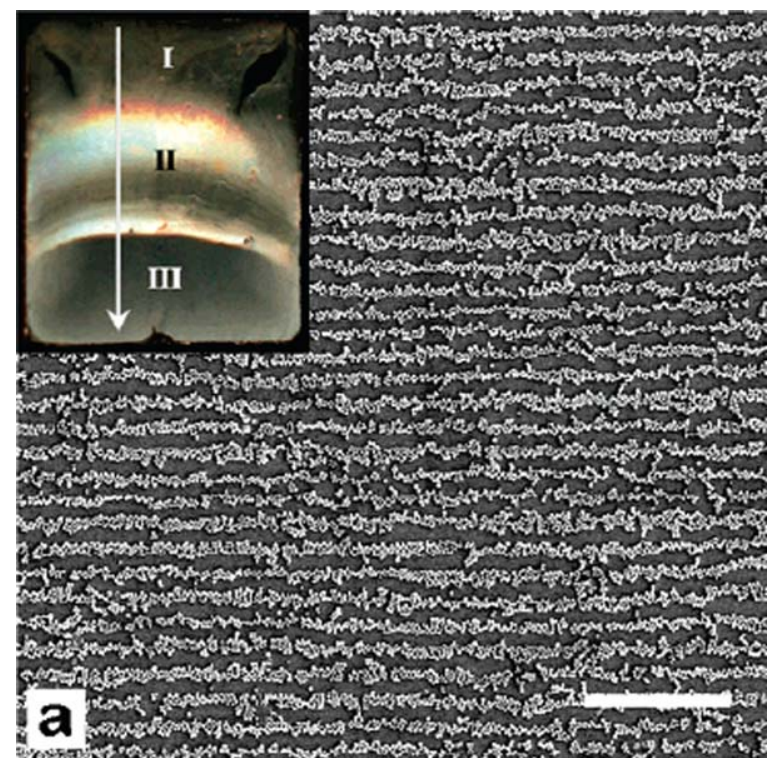

Figure 20. SEM image of periodic deposition pattern of $258 \mathrm{~nm}$ charged particles deposited on oppositely charged glass.

Scale bar: $10 \mu \mathrm{m}$. The inset $(12 \mathrm{~mm}$ wide) shows the deposition pattern over a large area, with three regions of deposition. The arrow indicates the liquid receding direction during the drying process. Region I contains an irregular deposition, while the periodic lines shown in the main figure appear in Region II. Region III contains more sparsely deposited particles (Reprinted with permission from Ray, Kim, and $\mathrm{Jia}^{63}$ Langmuir; 21:4786-4789, 2005. Copyright 2005 American Chemical Society). [Color figure can be viewed in the online issue, which is available at wileyonlinelibrary.com.] 

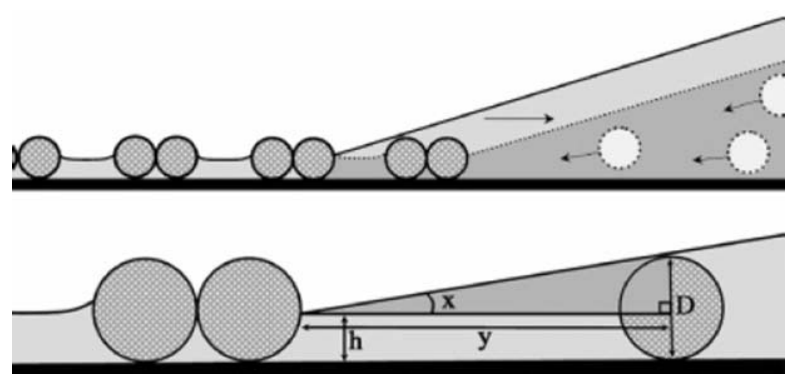

Figure 21. Top: Schematic of the process by which regularly spaced lines of colloids appear during droplet drying.

Bottom: illustration of the mechanism by which the line spacing is set. The line spacing is the distance y between the contact line and the closest approach to that line that a particle can attain without penetrating the free surface (Reprinted with permission from Ray, Kim, and Jia, ${ }^{63}$ Langmuir; 21:4786-4789, 2005. Copyright 2005 American Chemical Society).
Whether these conditions are met is sensitive to the type of solute or suspended particle, as is considered in the next sections.

\section{Colloidal and nanoparticle deposition}

For colloidal particles, the effect of suppression of evaporation near the droplet edge was investigated by Deegan et al., ${ }^{1}$ experimentally, by placing droplets in close proximity to each other, so that a higher concentration of droplet phase vapor in the space between the droplets might suppress evaporation there. The result was diminished deposition at the contact line in these regions, as expected. This phenomenon was leveraged by Chen and Evans ${ }^{61}$ to produce arched structures by drying arrays of highly concentrated suspensions of alumina powder (see Figure 19).

If the contact line does not remain pinned, deposition no longer accumulates at the droplet edge. Pinning and depinning can occur once, twice, or many times during the drying of a droplet, depending on the substrate, the particle type, and possibly the conditions of drying. In the case of only two pinning events during the course of drying, Nguyen et al. ${ }^{62}$ refer to a "late" pinning mode, to
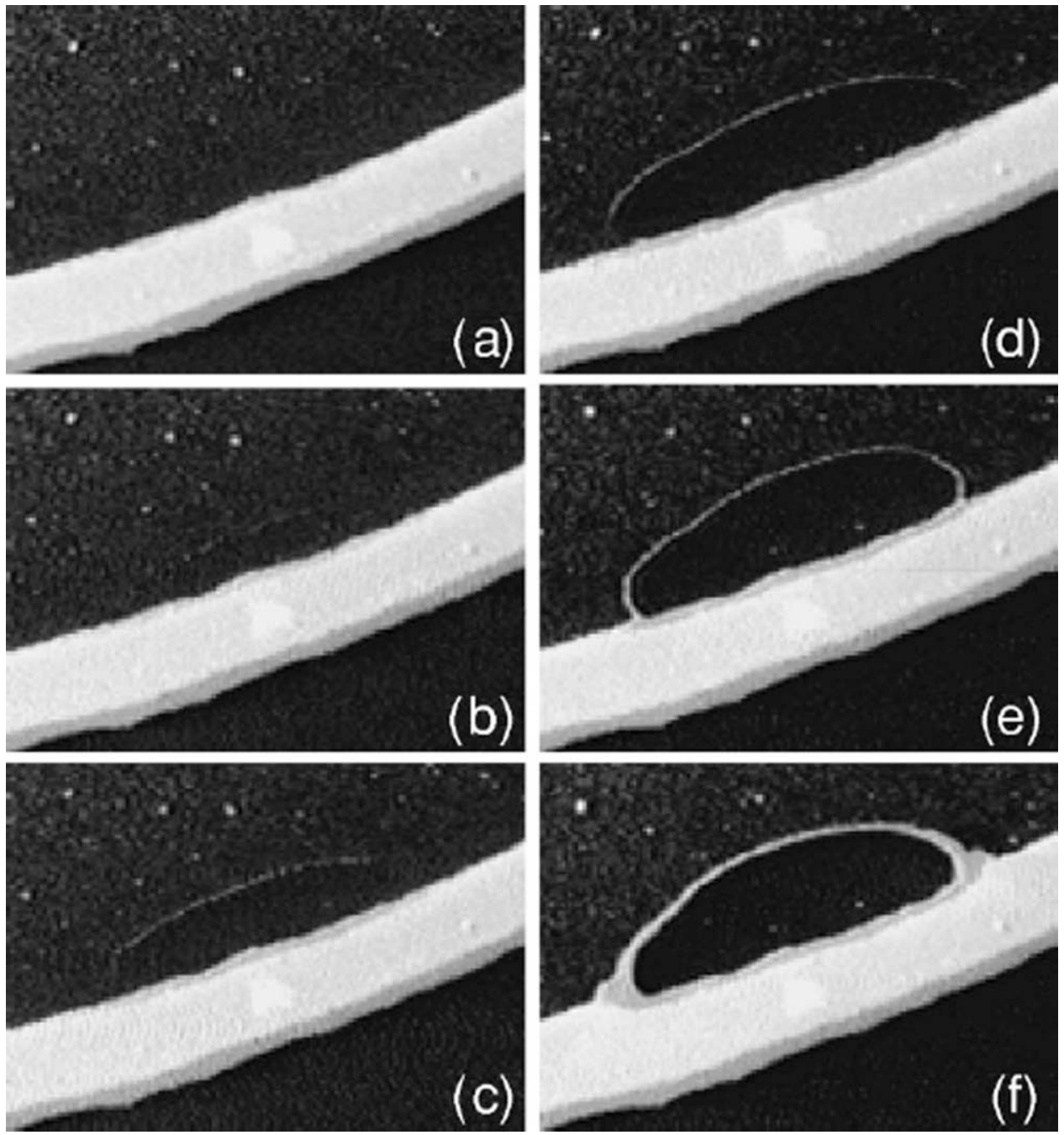

Figure 22. The formation of a secondary contact line deposit of micron-size polystyrene colloids around a dry patch from a water droplet on freshly cleaved mica.

The secondary deposit is the thin white line bounding the oval-shaped dark region, which is the dry patch, that is bounded on the other side by the outer contact-line colloidal deposit (the thick white line). Starting from time set to zero in (a) the time succession is (b) 0.23 , (c) 0.50 , (d) 0.83 , (d) 1.87 , and (f) $5.90 \mathrm{~s}$. The major axis of the dry patch is $150 \mu \mathrm{m}$ (from Deegan, ${ }^{2}$ Phys Rev E; 61:475-485, 2000. Copyright 2000 by the American Physical Society). 

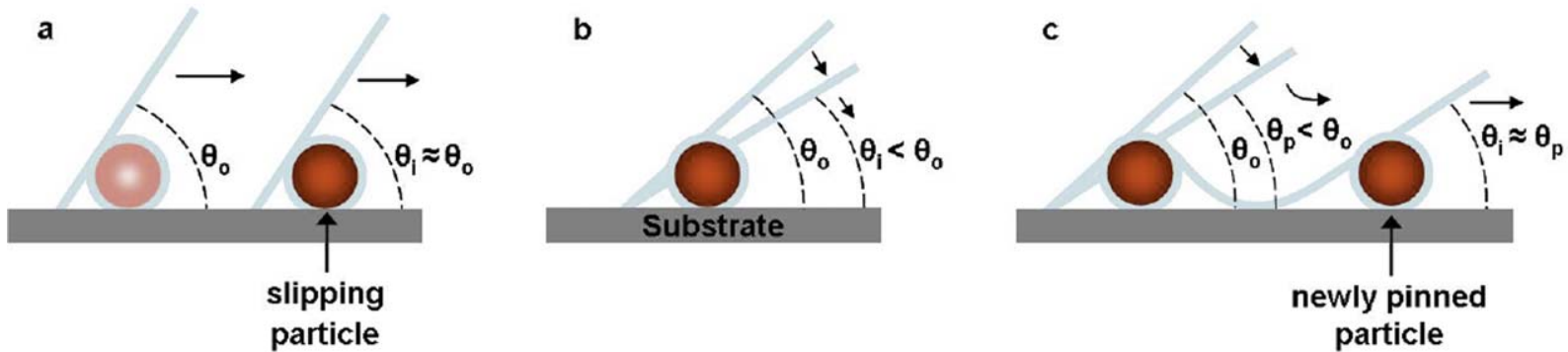

Figure 23. Schematic of contact line-colloid interactions at the air-water-solid interface during droplet evaporation.

(a) Slipping contact line mode, (b) pinned contact line mode, and (c) recurrent stick-slip contact line mode (Reprinted with permission from Morales, Parlange, Wu, Zhang, Sang, and Steenhuis, ${ }^{64}$ Langmuir; 29:1831-1840, 2013. Copyright 2013 American Chemical Society). [Color figure can be viewed in the online issue, which is available at wileyonlinelibrary.com.]

distinguish it from the first, or "early" pinning. An example of regular, periodic, pinning and depinning is depicted in Figure 20, which shows regular lines of charged colloids deposited onto an oppositely charge surface, at a contact line that periodically pins, depins and repins. The spacing between lines is insensitive to initial particle concentration, and is thought to be set by the closest proximity to the contact line attainable by an approaching particle without penetrating the free surface (see Figure 21). After a second line of particles forms behind the contact line, the contact line breaks free and retreats to this second line, as observed microscopically. ${ }^{63}$ An example in which this process occurs locally is shown in Figure $22 .^{2}$ Here, a dry patch nucleates behind the coffee ring, and particles accumulate along the inside edge of this patch, which pins the contact line there. Multiple events similar to this lead to irregular contact-line deposits.

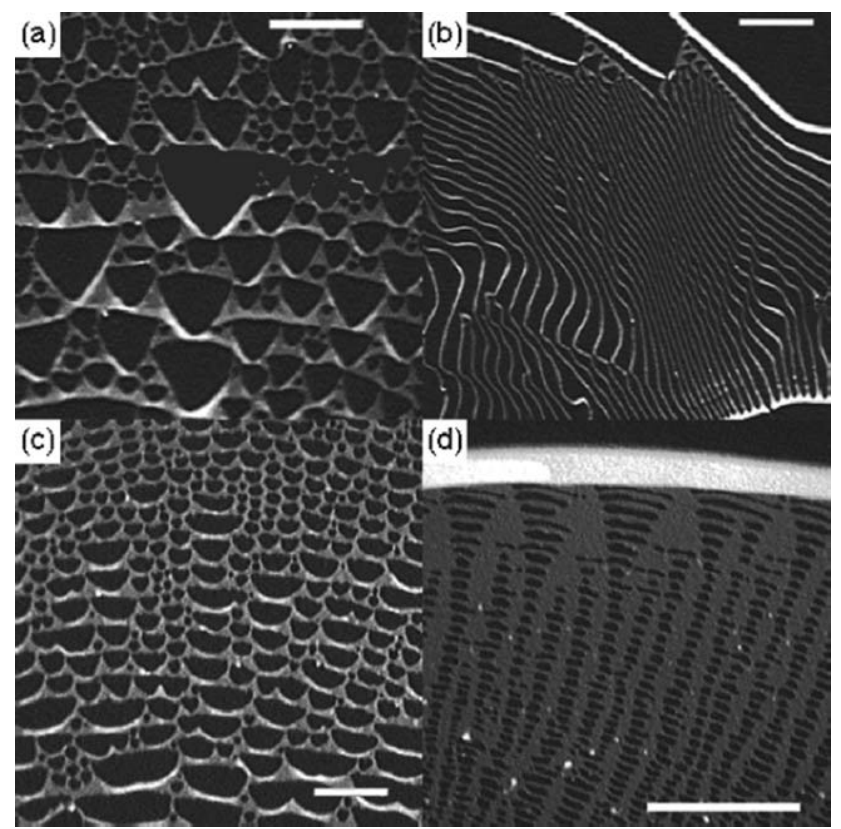

Figure 24. Deposit left by $0.1-\mu \mathrm{m}$ microspheres of $0.5 \%$ initial volume fraction when a SDS surfactant is added.

The center of the droplet lies below the image. In frames a-d, the concentration of surfactant is $0.81 \mathrm{mM}$, $0.43 \mathrm{mM}, 0.14 \mathrm{mM}$, and $0.048 \mathrm{mM}$, respectively. The scale bar corresponds to $50 \mu \mathrm{m}$ (from Deegan, ${ }^{2}$ Phys Rev E; 61:475-485, 2000. Copyright 2000 by the American Physical Society).
In the case just discussed, colloids, once deposited onto the substrate, do not move, although the contact line can move by breaking free of the deposited line of colloids. Another possibility is that the contact line might drag the particles with it, pulling them toward the center of the droplet. This has been observed by Morales et al. ${ }^{64}$ who manipulated the air-water surface tension and adhesion of $5 \mu \mathrm{m}$ particles to the substrate by addition of surfactant. Morales et al. ${ }^{64}$ developed a simple theory for this that balanced the frictional force resisting movement of the particles along the substrate against the component of capillary force pushing the particles toward the center of the droplet. The frictional force, inferred from the data using this balance, compared favorably to the attachment strength of the particles to the surface. This was measured by determining the speed of a stream of water, flowing parallel to the substrate, needed to wash off deposited particles from the surface. Attachment affinities in excess of above $0.32 \mathrm{mN}$ appeared to be necessary to pin the particles to the surface.

The particles deposited in the work of Morales et al. ${ }^{64}$ were observed to slip with the contact line along the surface at lowsurfactant concentration, to pin with the contact line at higher surfactant concentration, and for the contact line to break free of the pinned particles at still higher surfactant concentration, producing intermittent stick-slip motion of the contact line, but no sliding of deposited particles. These regimes, depicted in Figure 23, could be explained as follows. At low-surfactant concentration, the contact angle is high (Figure 23a), so that the component of the capillary force acting to press the particles normal to the surface is low. This produces a lowfrictional force resisting particle motion, according to Newton's law of friction (that frictional force is proportional to normal force). At higher surfactant concentration, the contact angle is smaller (Figure 23b) and the frictional force higher on the particles, pinning them to the contact line. As drying progresses, the pinned contact line develops an even smaller angle, and the deposited particles try to protrude from the droplet surface, producing a yet stronger pinning force. At still higher surfactant concentration, the air-water interface can expand and rupture (Figure 23c), leaving the outermost ring to dry out and the contact line to retreat until pinned again by new particles from the bulk.

Much more complex patterns are possible, include cellular and lamellar structures, sawtooth patterns, and "Sierpinski gaskets", depending on the pinning, depinning, and deposition dynamics (see Figure 24). ${ }^{2}$ Details regarding these and other patterns, scaling laws and descriptions of likely mechanisms can be found in Deegan. ${ }^{2}$ 


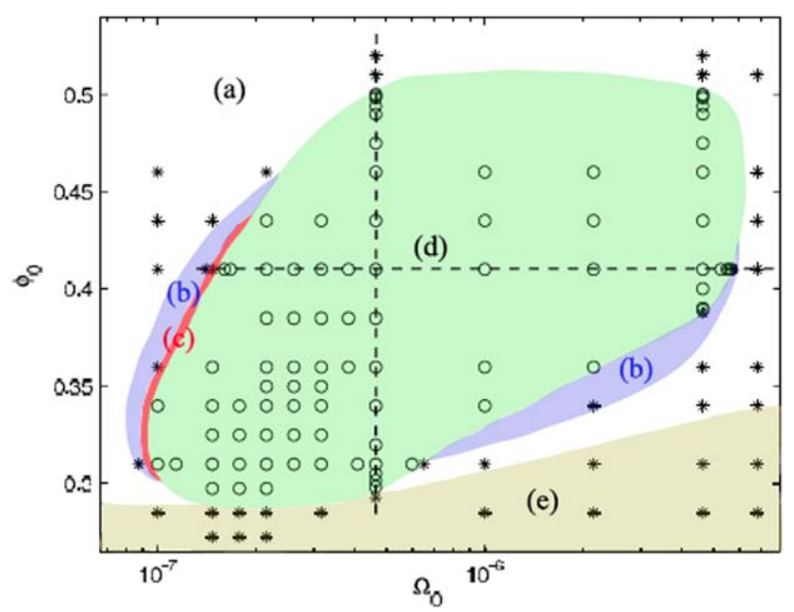

Figure 25. "Phase diagram" of deposition patterns, determined by thin-film evaporation number $\Omega_{0}$ and colloid volume fraction $\phi_{0}$.

Symbols denote simulation results. Region (a) contains a single line (or ring), (b) contains multiple lines, (c) is intermittent patterns, (d) contains regular periodic line patterns, and in (e) there are no lines (from Frastia, Archer, and Thiele, ${ }^{44}$ Phys Rev Letts, 106:077801, 2011. Copyright 2011 by the American Physical Society). [Color figure can be viewed in the online issue, which is available at wileyonlinelibrary.com.]
The first systematic attempt to develop a quantitative theory to predict which patterns of rings will result from pinning and depinning dynamics was made recently by Frastia et al. ${ }^{44}$ Their model is based on two coupled equations, a lubrication equation for hydrodynamics and a mass balance equation for the concentration of particles. The hydrodynamic equation includes a precursor film, the flow through which is governed by a thin-film van der Waals force, the strength of which is given by a Hamaker constant. The viscosity of the particle suspension follows the KriegerDougherty equation $\eta(\phi)=\eta_{0}(1-\phi)^{-v}$, where $\eta_{0}$ is the solvent viscosity and $v=1.575$. Thus, the suspension viscosity increases without bound as the particle concentration increases. A key parameter of the model is a thin-film evaporation number $\Omega_{0} \equiv 18 \beta \eta_{0} \gamma / \rho\left[6 \pi A^{2}\left|\tilde{S}^{P}\right|\right]^{1 / 3}$ (which differs from the evaporation number $\mathrm{E}$ defined earlier), where here $\beta$ is the evaporation rate, $\gamma$ the surface tension, $\rho$ the fluid density, A the Hamaker constant, and $\tilde{S}^{P}$ is a "spreading coefficient". See Frastia et al. ${ }^{44}$ for details. $\Omega_{0}$ is the ratio of the rate of evaporation to the rate of convection of the pure solvent in a thin film near the macroscopic contact line. An "effective evaporation number" is $\Omega_{0} \eta(\phi) / \eta_{0}$, which accounts for the viscosity of the solvent in the presence of particles. When the particle concentration is low, $\Omega_{0}$ is small, and convection is rapid. However, the concentration of particles in the thin film increases as evaporation occurs,
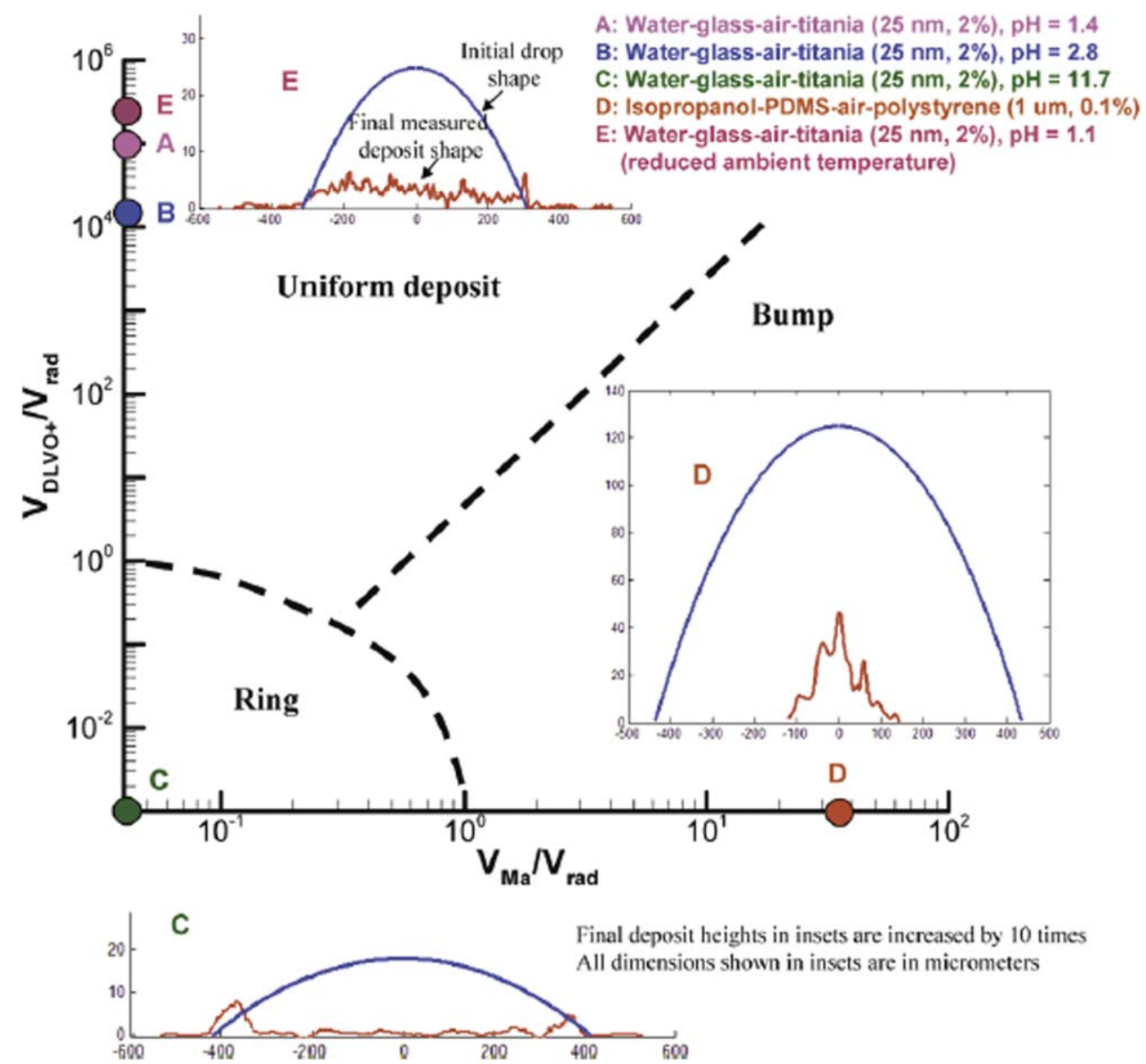

Figure 26. "Phase diagram" for self-assembly of nanoparticles during droplet drying on a solid surface. The two ratios of three characteristic velocities determines the deposition pattern.

Here $V_{\text {rad }} \sim U_{M L}$ is the magnitude of the radial flow velocity caused by evaporation, $V_{\text {DLVo }}$ is the velocity caused by an attractive DLVO force (electrostatics plus van der Waals), and $V_{\mathrm{Ma}} \sim U_{\mathrm{ML}} M a_{U}$ is the Marangoni velocity scale (Reprinted with permission from Bhardwaj, Fang, Somasundaran, and Attinger, ${ }^{65}$ Langmuir; 26:7833-7842, 2010. Copyright 2010 American Chemical Society). [Color figure can be viewed in the online issue, which is available at wileyonlinelibrary.com.] 


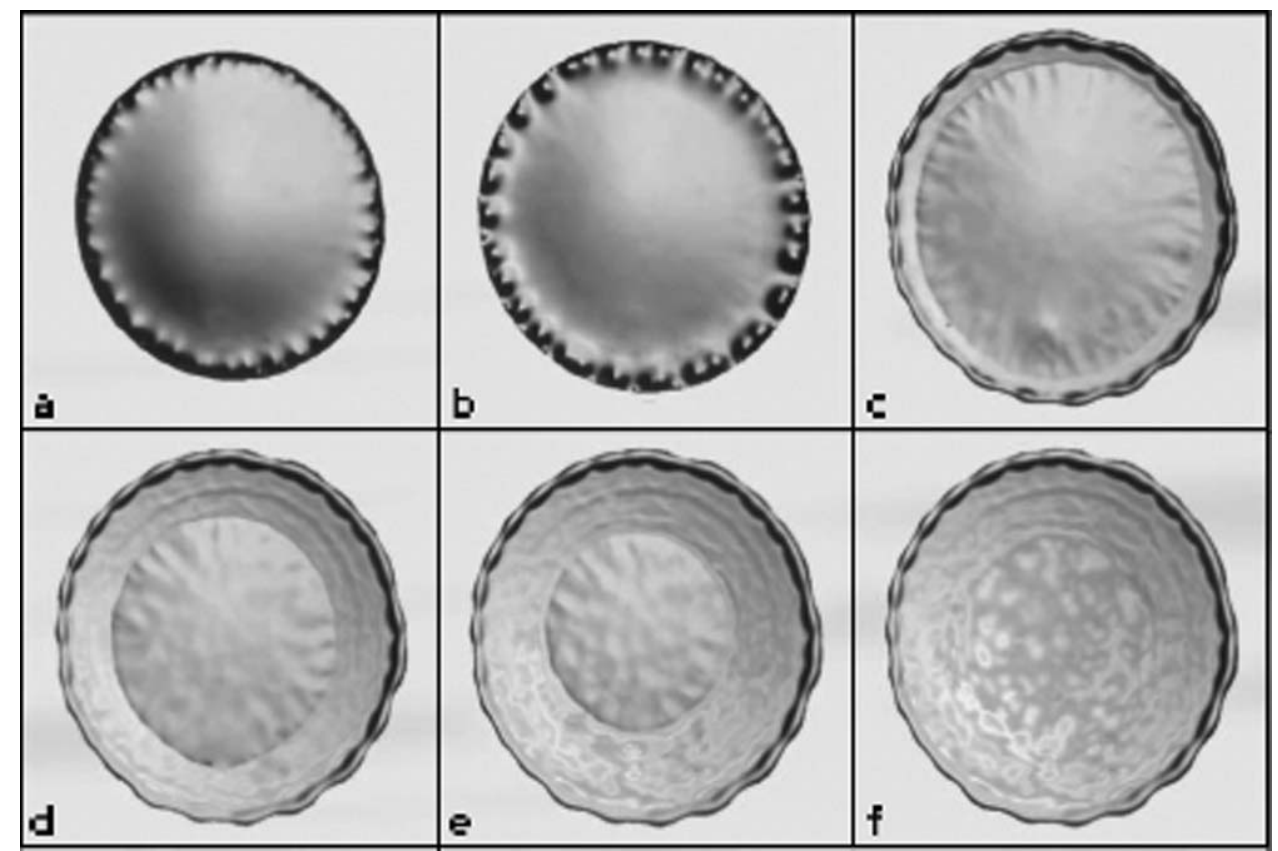

Figure 27. Evolution of shape of drying droplet of Heptane containing 5\% PDMS.

(a) An instability is during the spreading phase, leading to (b) roll formation at the contact line when the droplet radius reaches a maximum. (c,d,e) Receding phase with cells due to Marangoni flow, leaving (f) after complete drying (from Poulard and Damman, ${ }^{53}$ Europhys Lett; 80:64001, 2007, with permission from Europhyics Letters).

leading eventually to a large rise in viscosity and in the effective evaporation number, so that the suspension becomes hydrodynamically jammed, and forms a pinned line of solute. Eventually the fluid behind this line retreats from it and additional lines might form. A variety of scenarios are possible, as controlled by the particle concentration and the evaporation number, as diagrammed in Figure 25. These predictions seem not to have been tested yet, and it is possible that other phenomena, including thermal effects, more complex wetting effects, etc., might need to be included in the theory to make it realistic. However, these initial results suggest that theoretical progress is possible, even for such a complex problem as contact line deposits.

Pinning of the contact line does not guarantee that a "coffee ring" deposit will occur at the contact line. A strong electrostatic attraction of the particles to the surface can induce deposition of the particles before they reach the contact line. ${ }^{65}$ In addition, a Marangoni flow can sweep the particles toward the center of the droplet, causing deposition there. ${ }^{17}$ A "phase diagram" showing these three possibilities as functions of particle velocities induced by attraction to the surface, radial outflow due to evaporation, and Marangoni velocity, are shown in Figure 26. In mixed colloidal systems, with some particles well dispersed and some not, the Marangoni flow can keep the well dispersed particles in suspension, while the less-well dispersed ones aggregate at the contact line. The result is a flow-induced demixing of complex particle mixtures during drying, a phenomenon of some consequence for printing and drying of inks, from inkjet printers, for example. ${ }^{66}$

A uniform deposition pattern on a variety of substrates can also be produced by drying water droplets in an ethanol vapor, whose adsorption reduces the contact angle, and induces droplet spreading and Marangoni flow. ${ }^{67}$ Uniform deposition of a monolayer of hexagonally ordered 6-nm spherical dodecanethiol-passivated gold nanocrystals was obtained by
Bigioni et al. ${ }^{68}$ The process for obtaining this involved diffusion of the small gold nanoparticles to the liquid-air interface, where they assembled into rafts, which resisted convection to the contact line. Instead, the rafts were driven together during late-state drying, as the contact line depinned and the droplet shrunk in radius. At the end, a single large raft, containing as many as $10^{8}$ particles was laid down onto the substrate. Rapid evaporation was observed to be important to this process. The opposite effect of evaporation speed was observed by Marín et al. ${ }^{69}$ for micron-size spheres, which ordered into a hexagonal phase particles on the substrate if they arrived slowly enough at the contact line during droplet drying. If they arrived at a rate too fast for them to rearrange into hexagonal order, they formed a "glassy" deposit at the contact line. The velocity of arrival below which the ordered phase formed is apparently set by the ratio of the time between successful arrivals of new particles to a position along the contact line to the time required for particles to rearrange by diffusion near the contact line. ${ }^{69}$ Consistent with this, Yan et al. ${ }^{70}$ showed that by changing the charge of the particles relative to that of the substrate, or by addition of surfactant, the mobility of the particles at the contact line could be increased or decreased, thereby controlling whether ordered or disordered particle assemblies formed. For example, particles of charge opposite that of the surface were relatively immobile and formed a disordered deposit, while the opposite occurred for particles of the same charge as the substrate.

Yet another way of obtaining more uniform deposition is to use elongated micron-size particles, with aspect ratio of 1.2 or greater, as revealed in a series of intriguing experiments by Yunker et al. ${ }^{71}$ As with the gold nanoparticles studied by Bigioni et al. ${ }^{68}$ Yunker et al. observed that the elongated colloidal particles on the air-liquid interface resist convection to the contact line, resulting in more uniform particle deposition. For the elongated colloidal particles, this resistance is apparently due to the strong deformation of the 
a)

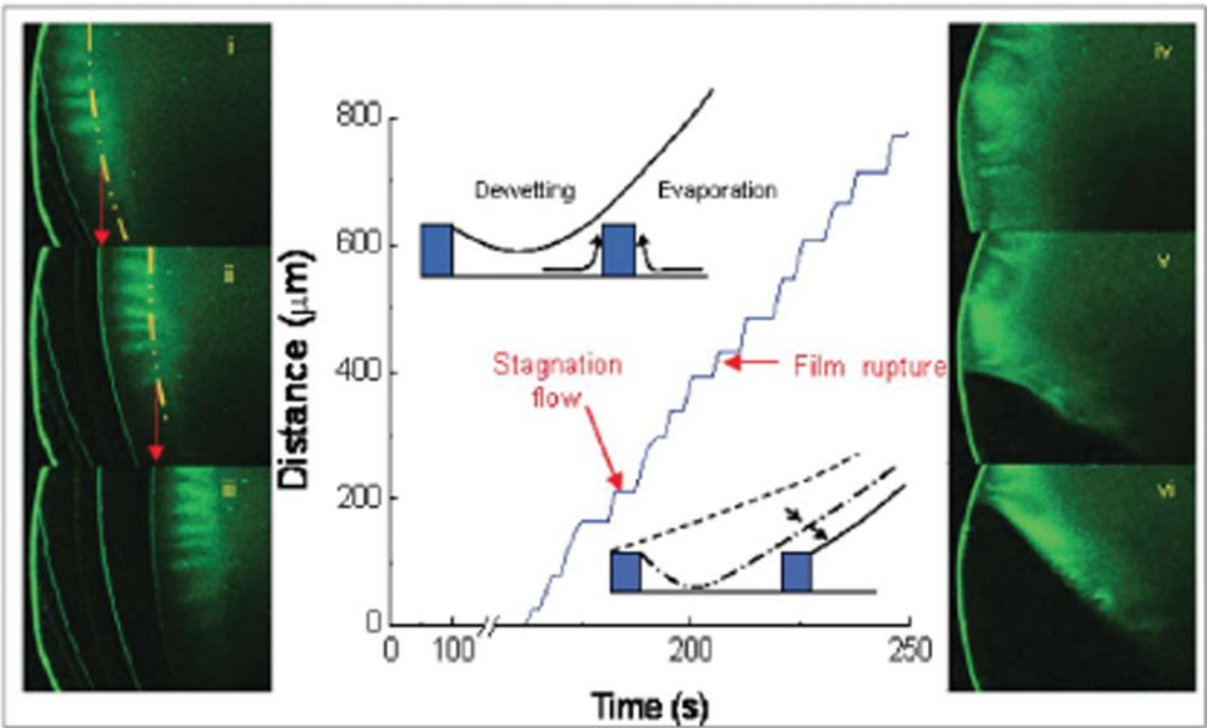

b)
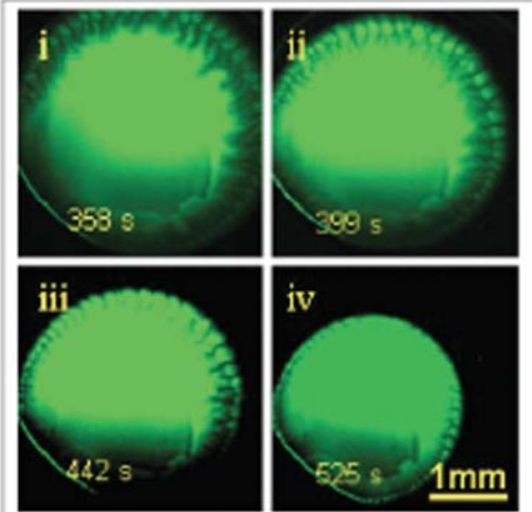

c)

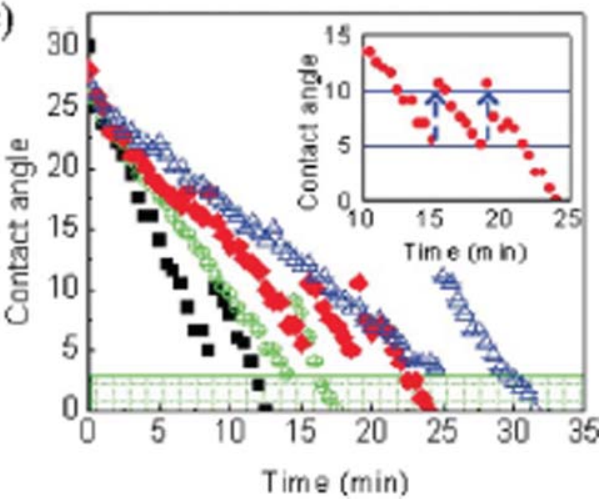

Figure 28. Drying of a droplet containing green-stained DNA.

(a-left): Images of receding dynamics for the contact line, in time series from top to bottom. (a-center) Depiction of stagnation flow leading to buildup of deposit behind the contact line, and "staircase" pattern of contact line position as a function of time, due to pinning and depinning cycles. The horizontal part of the staircase indicates pinned contact line, when the stagnation flow is creating the internal ring. (a-right): Rupture of film between contact line and inner ring in time sequence from top to bottom. (b-left) Viscous fingering pattern in a drop with DNA concentration $50 \mu \mathrm{g} / \mathrm{ml}$. (c) Contact angle variation during typical evaporation sequence, for different drop volumes: (black) $4 \mu \mathrm{l}$, (green) $8 \mu \mathrm{l}$, (red) $12 \mu \mathrm{l}$, and (blue) $16 \mu \mathrm{l}$. The shaded portion near the time axis represents uncertainty in measurement due to very small angles. The inset shows the cyclic variation in contact angle, due to pinning and depinning (from Maheshwari, Zhang, Zhu, Chang, ${ }^{82}$ Phys Rev Lett; 100:044503, 2008. Copyright 2008 by the American Physical Society).

liquid-air interface that occurs near elongated particles, causing them to repel each other and avoid pile-up at the contact line. The deposit that does form at the contact line is also affected strongly by particle aspect ratio, with variations in deposit height along the droplet periphery being especially sensitive to particle aspect ratio. ${ }^{72}$ The variety of ways of forming more uniform deposits suggests the importance of the interaction of convection, Brownian motion, and a variety of liquid-air-particle and liquid-substrate-particle interactions that are not fully understood.

At high-volume fractions of latex particles, the "crust" that forms on the free surface during drying can cover the whole droplet surface and become unable to shrink its area to comply with the shrinking droplet. It may, therefore, buckle inward, leading to a dimple at the top of the droplet, which can under some conditions grow into a hole, leading to a toroidal deposit. ${ }^{73}$ For similar reasons dimple can also form during drying of a semicylindrical 2-D linear "droplet" containing a concentrated colloidal suspension, used for example in printing. ${ }^{74}$
Other complex deposition patterns can be formed by drying of droplets containing anisotropic particles or mixtures of particles. Vakarelski and coworkers ${ }^{75}$ formed gold nanoparticle wires on substrates, using deposited large particles for templates around which gold nanoparticles assembled into wires. Strano and coworkers ${ }^{76}$ found that suspended nanotubes would align when dried onto a substrate from a droplet. If the nanotubes are attached at one end to the surface and aligned vertically in a dense array, the flow during drying can organize them into foam-like cellular patterns. ${ }^{77}$ Harris et al. ${ }^{78}$ were able to pattern colloidal deposition into regular arrays of spots using a mask over the droplet to impose a regular spatial evaporation pattern. This led to a similarly patterned Marangoni flow, which then transferred this pattern to the colloidal deposition, leading to a beautifully regular array of colloidal deposits. Another way to control colloid deposition is through use of surfactants, which coat the droplet surface and can undergo phase transitions as the droplet dries, which in turn can influence Marangoni convection and the resulting colloid deposition pattern, 


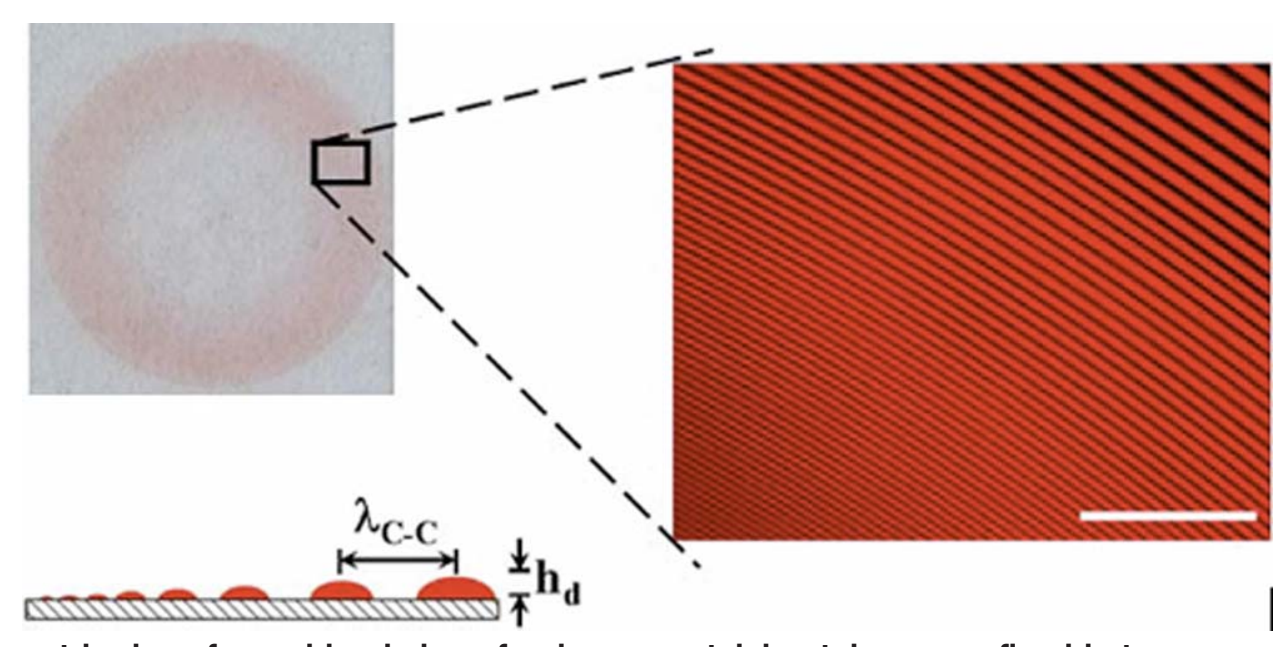

Figure 29. Concentric rings formed by drying of polymer-containing toluene confined between a sphere and a flat surface.

The right side shows a magnified zone (scale bar is $200 \mu \mathrm{m}$ ). As the solution front moves inward, the rings become smaller and their height decreases as illustrated in lower left (from Xu, Xia, Hong, Lin, Qiu, and Yang, ${ }^{83}$ Phys Rev Lett; 96:066104, 2006. Copyright 2006 by the American Physical Society). [Color figure can be viewed in the online issue, which is available at wileyonlinelibrary.com.]

producing, for example, polygonal networks of deposits. ${ }^{79}$ Yet another way of organizing complex patterns is through mixing polymers with colloids. Under conditions where colloid phase separation is induced by the polymer, Senses et al. ${ }^{80}$ observed Marangoni eddies near the pinned contact line, producing radial stripes of colloidal particles.

\section{Polymer deposition}

When polymer or protein is the solute in a drying droplet containing volatile solvent, some additional interesting features of dried deposits are revealed. An example is shown in Figure 27, depicting the drying of a heptane droplet containing $5 \%$ polydimethylsiloxane (PDMS). In this case, the surface tension of the polymer exceeds that of the solvent. Hence, near the droplet edge, where evaporation increases the polymer concentration, the surface tension is elevated, and Marangoni flow drags fluid to the rim. This leads to a region of inverse curvature near the rim, producing a "Mexican hat" shape. ${ }^{53}$ A similar shape has been observed for the drying of protein solutions, and predicted by an analysis that accounts for the large viscosity enhancement produced by the increased protein concentration at the edge. ${ }^{81}$ For the polymer solution depicted in Figure 27, the rippling of the bulge at the rim is evidently produced by a Marangoni instability, made possible by the thickening of the film at the rim, combined with the concentration-dependent surface tension.

For drying of colloid-containing droplets, in Figure 20, an example of regular colloid line deposits was shown, produced by cyclic pinning and depinning of the contact line. A counterpart to this for polymers is the regular line formation is depicted in Figure 28a (left), which is a series of rings formed sequentially as the result of periodic pinning and depinning of a contact line of a droplet containing stained DNA molecules (in green). ${ }^{82}$ The hypothesized mechanism is a stagnation flow behind the contact line that concentrates solute (DNA) at the stagnation ring. One can see this enhanced concentration of DNA behind the series of rings on the left side of Figure 28a. As depicted in the center sketch of Figure 28a, this leads to building of a deposit at an inner ring behind the contact. The film of fluid between the contact line and the inner ring then ruptures as shown in Figure 28a (right). There is also a viscous fingering pattern near the outer ring, which is

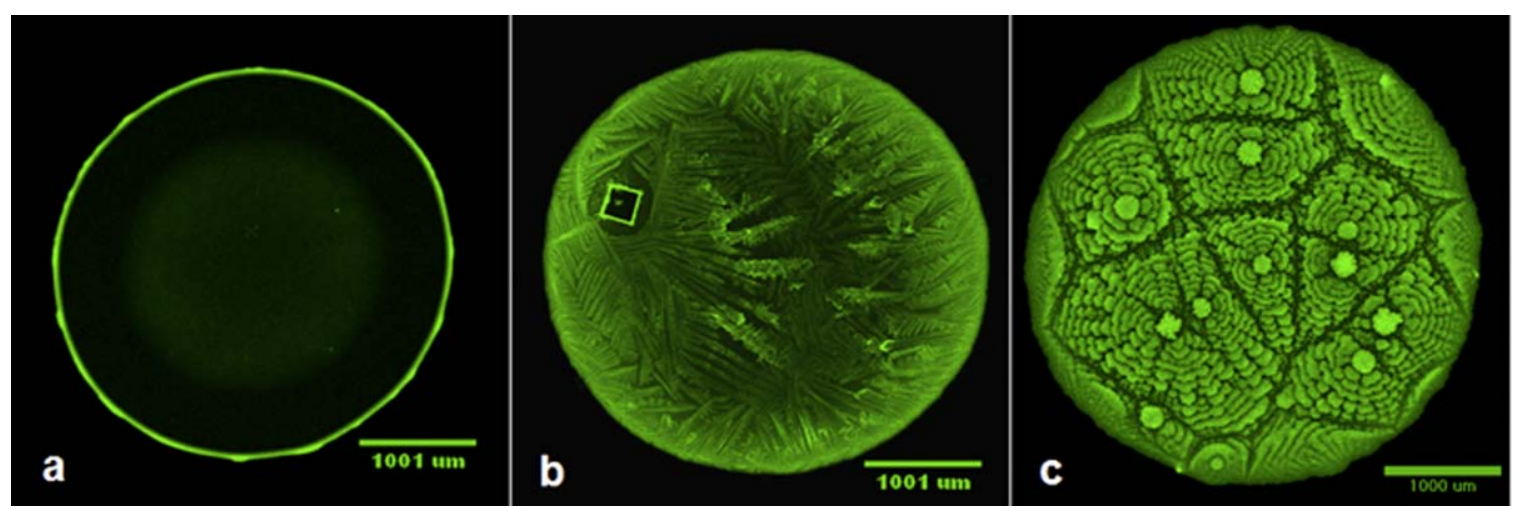

Figure 30. Deposition patterns on glass from a drying aqueous droplet with $0.01 \%$ fluorescently stained albumin at various buffer compositions (a) $\mathrm{NH}_{4} \mathrm{HCO}_{3}, 0.1 \mathrm{M}, \mathrm{pH}=8.40$, (b) phosphate buffered saline, $0.01 \mathrm{M}$, $\mathrm{pH}=7.47$, and (c) $\mathrm{NaHCO}_{3}, 0.1 \mathrm{M}, \mathrm{pH}=8.45$.

(Scale bars are $1000 \mu \mathrm{m}$. The square on the left side of the image in (b) is a salt crystal decorated with stained protein (Reprinted with permission from Larson, Lopez, Lim, and Lahann, ${ }^{90}$ MRS Proceedings. 2010;1273:1273-MM03-01. Copyright 2010, Materials Research Society). [Color figure can be viewed in the online issue, which is available at wileyonlinelibrary.com.] 

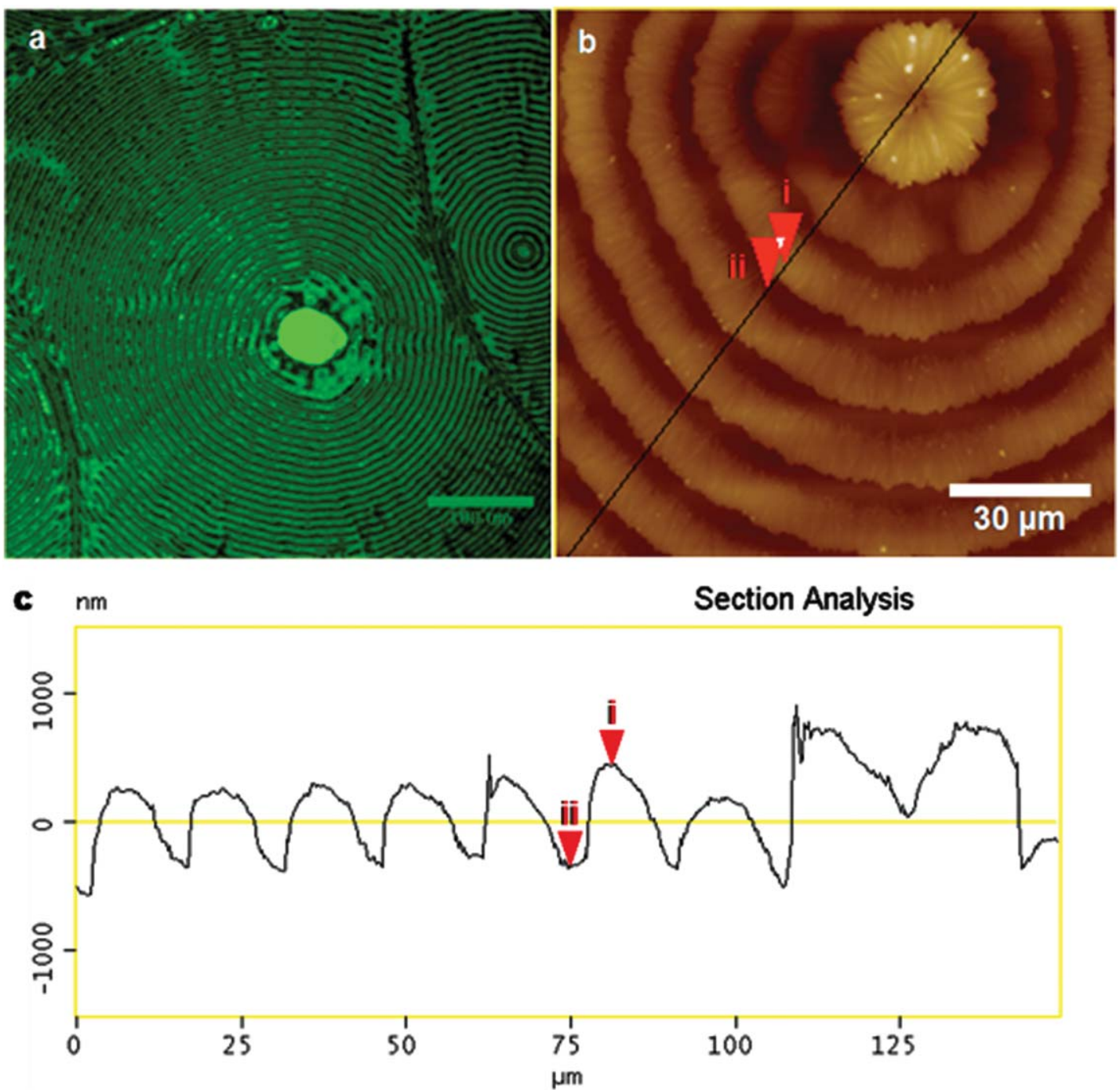

Section Analysis 
(a)

(c)
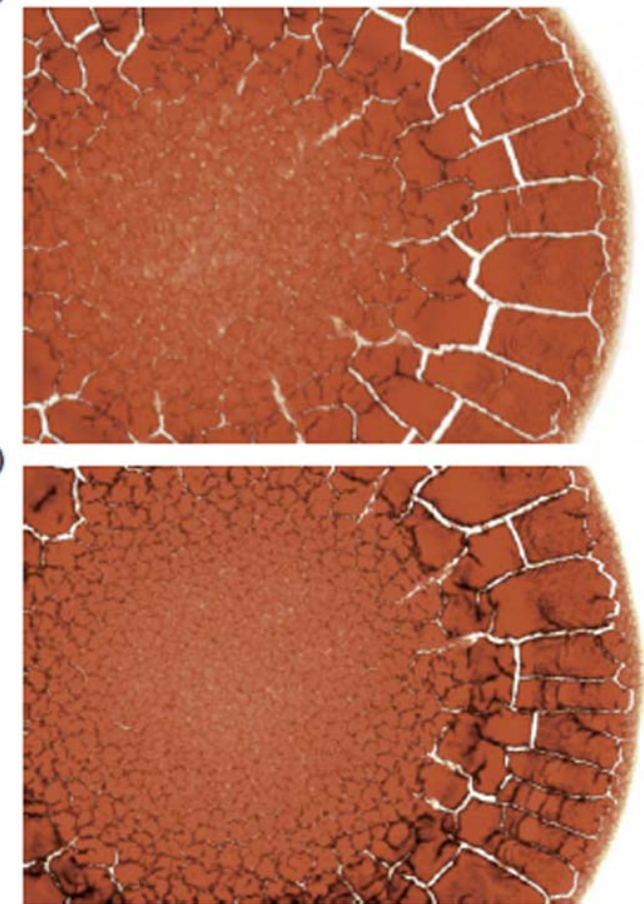

(b)

(d)
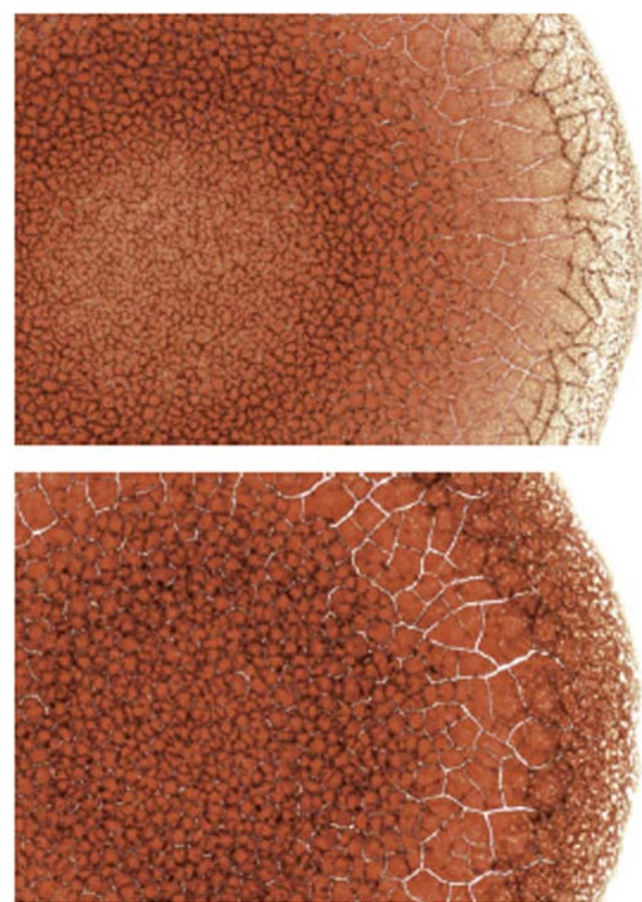

Figure 32. Drops of blood from individuals (a) in good health, (b) with anemia, (c) in good health, and (d) with hyperlipidemia (from Brutin, Sobac, Loquet, and Sampol, ${ }^{97} \mathrm{~J}$ Fluid Mech; 667:85-95, 2011, with permission from Cambridge University Press).

[Color figure can be viewed in the online issue, which is available at wileyonlinelibrary.com.]

an unusual "soccer-ball" pattern. Other patterns observed include concentric rings and triangular crystals. ${ }^{89}$ Higher resolution images, including an atomic force microscopy image, of the "soccer-ball" pattern are displayed in Figure 31. Note that the pattern in Figure 30c corresponds to densepacked polygonal regions containing periodic, or wave-like, deposition patterns. Such crystal patterns are sensitive to the type and concentration of solutes, the substrate wettability, and other factors. ${ }^{89-92}$ The nonuniformity of the deposition is likely related to the super-saturation needed to rapidly nucleate crystals. Once crystallization starts, it likely proceeds rapidly enough to deplete nearby liquid of crystalforming solutes, thus, shutting off deposition over a distance scale set by the distance such salts can diffuse within the available time. A theory that successfully predicts concentric ring spacing for deposited crystals ${ }^{89}$ shows that the spacing is proportional to $\frac{D \rho_{L}}{D_{\text {vap }}(1-H) \rho_{\text {vap }}} \theta_{0} R \propto V_{\text {drop }} \phi_{0} / R^{2}$ where $D$ is the diffusivity of the salt, $\rho_{L}$ is the liquid density, $\theta_{0}$ is the initial contact angle, $R$ is the droplet radius, $D_{\text {vap }}$ is the diffusivity of the water vapor, $H$ is the humidity far from the droplet, $\rho_{\text {vap }}$ is the saturation vapor pressure of water, $V_{\text {drop }}$ is the droplet volume, and $\phi_{0}$ is the initial salt concentration. This quantity is a length-scale that reflects the competition between the rate of diffusion of salt to the rate of volume shrinkage due to evaporation.

The dendritic patterns observed in Figure 30b are common to crystallization in general, such as the ubiquitous snowflake pattern. This dendritic growth occurs because protrusions from a crystal surface, such as corners, stick farther into the solute field, and, hence, experience a steeper concentration gradient than do surrounding areas. Hence, such protrusions tend to grow, which enhances further the concentration gradient, leading to still more growth, and additional protru- sions. This mechanism, known as the Mullins-Sekerka instability, ${ }^{92,93}$ is the solid-phase counterpart of a fingering instability in liquids. The sensitivity of the pattern formed to the type and concentration of solute suggests that deposition patterns might be used as easily obtained "fingerprints" for disease markers in blood or saliva. ${ }^{94}$

\section{Applications to disease detection}

The possibility that strain patterns left by the drying of biological fluids such as blood or saliva could be the basis of low-cost, low-resource disease diagnostics has been recognized, especially in recent years. ${ }^{94-97}$ Figure 32 shows an example in which stains from blood from two individuals in good health (a and c) are compared to those from diseased individuals ( $b$ and $d$ ). In low-resource environments, such simple tests could quickly determine which individuals need further testing, and even possibly suggest the likely disease. A more precise test for a specific disease was developed very recently by Tantrum et al. ${ }^{95}$ who showed that a droplet containing magnetic nanoparticles and a fluorescently labeled micron-sized particles could form a ring and other deposition patterns that were sensitive to the presence of poly-Lhistidine, a peptide mimic of a malaria biomarker. Given the prevalence and devastation wrought by malaria in underdeveloped countries, such a test, if made inexpensive and reliable, could be a life-saver. Recent work by Wen et al. ${ }^{96}$ shows the possibility of detecting another protein, $\alpha$ thrombin, in human serum using aptamer beacons to produce fluorescence signals sensitive to the presence of the protein. To make such methods useful in an environment where skilled workers are scarce, a method of automating such tests would be highly desirable, and, in fact, a method of computer-automated recognition of stain patterns has also 
been recently proposed and demonstrated by Kim et al. ${ }^{98}$ Further developments in this fast-moving area are likely in the near future.

\section{Summary and Concluding Remarks}

I have reviewed the very rich phenomena involved in drying droplets. The phenomena cover a wide swath of transport phenomena, including momentum, heat, and mass transfer, both in the linear and nonlinear regimes, including free surfaces, and moving contact lines. In addition, colloidal and polymer dynamics, wetting effects, and adhesion play starring roles. Applications range from printing to disease diagnosis. Despite the complexity of the phenomena, theoretical and even analytical predictions are possible in some simple, but realizable, cases. A pinned contact line in a sphericalcap droplet with one-way coupling of transport phenomena is one such case. The analysis of this case by Deegan et al. ${ }^{1}$ in 1997 set off a flurry of work on the drying droplet problem that continues to this day.

Given the range of phenomena that I have reviewed, it is difficulty to draw general conclusions. However, it is probably fair to say that understanding of the continuum heat, mass, and momentum transport phenomena for singlecomponent fluids is relatively well developed, while the interfacial, contact-line, and deposition processes in drying droplets are not nearly as well understood. The literature on the latter, while rich and fascinating, is still rather disjointed. While mechanisms are often given for observed deposition patterns, follow-up studies to test these mechanisms by varying the type, size, or concentration of particle or polymer, or the effect of substrate or fluid properties, are rare. Thus, a truly systematic, well-established, body of knowledge on deposition from drying droplets is, for the most part, not yet available. Nevertheless, some common features of deposition patterns are now evident, including single and multiple rings, regularly spaced ring patterns, and dendritic patterns. At least two mechanisms for formation of regularly spaced rings have been identified, one involving periodic contact-line pinning and depinning, and the other involving diffusion-limited deposition. It is encouraging that these periodic patterns can be observed in colloidal, polymeric, and crystallizing deposits, and similar mechanisms likely underlie all of them. In addition, detailed theories, using coupled field equations, have emerged that are predictive to some extent, including equations that describe nanoscale wetting effects.

Thus, there is good reason to believe that significantly better understanding is possible. It is hoped that this review article will more clearly identify where progress is most needed, and most readily attained. In particular, it is hoped that future work will not only seek to identify new phenomena, but that, in addition, careful work will be done to test and refine the models and ideas that have already been put forth. In this way, we may develop a more comprehensive understanding and a greater ability to exploit the wealth of phenomena present in the drying droplets and in the deposition patterns they leave behind.

\section{Literature Cited}

1. Deegan RD, Bakajin O, Dupont TF, Huber G, Nagel SR, Witten TA. Capillary flow as the cause of ring stains from dried liquid drops. Nature. 1997;389:827-829.

2. Deegan RD. Pattern formation in drying drops. Phys Rev E. 2000; 61:475-485.
3. Hu H, Larson RG. Evaporation of a sessile droplet on a substrate $J$ Phys Chem. 2002;106:1334-1344.

4. Birdi KS, Vu DT, Winter AJ. A study of evaporation rates of small water drops placed on a solid-surface. J Phys Chem. 1989;93:37023703.

5. Birdi KS, Vu DT. Wettability and the evaporation rates of fluids from solid surface. J Adhesion Sci Technol. 1993;7:485-493.

6. Rowan SM, Newton MI, McHale G. Evaporation of microdroplets and the wetting of solid surfaces. J Phys Chem. 1995;99:1326813271.

7. Rowan SM, McHale G, Newton MI, Toorneman M. Evaporation of microdroplets of three alcohols. J Phys Chem B. 1997;101:1265-1267.

8. Lugg GA. Diffusion coefficients of some organic and other vapors in air. Anal Chem. 1968;40:1072-1077.

9. Lebedev NN. Special Functions and Their Application.: Englewood Cliffs, New J: Prentice-Hall; 1965.

10. Picknett RG, Bexon R. The evaporation of sessile or pendant drops in still air. J Coll Interf Sci. 1977;61:336-350.

11. Widjaja E, Harris MT. Numerical study of vapor phase-diffusion driven sessile drop evaporation. Comput Chem Eng. 2008;32:21692178.

12. Hu H, Larson RG. Analysis of the effects of Marangoni stresses on the micro-flow in an evaporating sessile droplet. Langmuir. 2005;21: 3972-3980.

13. Fischer BJ. Particle convection in an evaporating colloidal droplet. Langmuir. 2002;18:60-67.

14. Masoud H, Felske JD. Analytical solution for Stokes flow inside an evaporating sessile drop:spherical and cylindrical cap shapes. Phys Fluids. 2009;21:042102.

15. Petsi AJ and Burganos AV. Stokes flow inside an evaporating liquid line for any contact angle. Phys Rev E. 2008;78:036324.

16. Tarasevich YY. Simple analytical model of capillary flow in an evaporating sessile drop. Phys Rev E. 2005;71:027301.

17. Hu H, Larson RG. Marangoni effect reverses coffee-ring depositions, J Phys Chem B. 2006;110:7090-7094.

18. Karapetsas G, Matar OK, Valluri P, Sefiane K. Convective rolls and hydrothermal waves in evaporating sessile drops. Langmuir. 2012; 28:11433-11439.

19. Ajaev, V. Spreading of thin liquid droplets on uniformly heated substrates. J Fluid Mech. 2005;528:279-296.

20. Maki K, Kumar, S. Fast evaporation of spreading droplets of colloidal suspensions. Langmuir. 2011;18:11347-11363.

21. Savino R, Fico S. Transient Marangoni convection in hanging evaporating drops. Phys Fluids. 2004;16:3738-3754.

22. Kang KH, Lee SJ, Lee CM, Kang IS. Quantitative visualization of flow inside an evaporating droplet using the ray tracing method. Meas Sci Technol. 2004;15:1104-1112.

23. Ristenpart WD, Kim PG, Domingues C, Wan J, Stone HA. Influence of substrate conductivity on circulation reversal in evaporating drops. Phys Rev Lett, 2007;99:234502.

24. Pearson JRA. On convection cells induced by surface tension. $J$ Fluid Mech. 1958;4:489-500.

25. Sobac B, Brutin D. Thermocapillary instabilities in an evaporating drop deposited onto a heated substrate. Phys Fluids. 2012;24:032103.

26. Duan F, Badam VK, Durst F, Ward CA. Thermocapillary transport of energy during water evaporation. Phys Rev E. 2005;72: 056303.

27. Barash, LYu, Bigioni TP, Vinokur VM, Shchur LN. Evaporation and fluid dynamics of a sessile drop of capillary size. Phys Rev E. 2009; 79:046301.

28. Barash LYu. private communication (2013).

29. Kelly-Zion PL, Pursell CJ, Vaidya S, Batra J. Evaporation of sessile drops under combined diffusion and natural convection. Coll Surf A: Physicochem Eng Aspects. 2011;1-3:31-36.

30. Duan F, Ward, CA, Badam VK, Durst F. Surface excess properties from energy transport measurements during water evaporation. Phys Rev E. 2005a;72:056302.

31. Duan F, Ward, CA, Badam VK, Durst F. Role of molecular phonons and interfacial temperature discontinuities in water evaporation. Phys Rev E. 2008;78:041130.

32. Persad AH, Ward CA. Statistical rate theory examination of ethanol evaporation. J Phys Chem B. 2010;114:6107-6116.

33. Ghasemi H, Ward CA. Energy transport by thermocapillary convection during sessile-waterdroplet evaporation. Phys Rev Letts. 2010; 105:136102.

34. Oron A, Davis SH, Banko SG. Long-scale evolution of thin liquid films. Rev Mod Phys. 1997;69:931-980. 
35. Semenov A, Starov VM, Rubio RG, Velarde MG. Computer simulations of evaporation of pinned sessile droplets: influence of kinetic effects. Langmuir. 2012;28:15203-15211.

36. Burelbach JP, Bankoff SG, Davis SH, Nonlinear stability of evaporating/condensing liquid films. J. Fluid Mech. 1988;195:463-494.

37. Xu X, Luo J, Guo D. Criterion for reversal of thermal Marangoni flow in drying drops. Langmuir. 2010;26:1918-1922.

38. Dunn GJ, Wilson SK, Duffy BR, Sefiane K. Evaporation of a thin droplet on a thin substrate with a high thermal resistance. Phys Fluids. 2009;21:052101.

39. Bird RB, Stewart WE, Lightfoot EN. Transport Phenomena. New York, NY: J Wiley \& Son; 1960

40. Hu H, Larson RG. Drying a sessile droplet: imaging and analysis of transport and deposition patterns. In: Lin Z, ed. Evaporative SelfAssembly of Ordered Complex Structures. Singapore: World Scientific Publishing Co; 2011. Note that there is a sign error in Eq. 54.

41. Xu X, Luo J. Marangoni flow in an evaporating water droplet. Appl Phys Letts. 2007;91:124102.

42. Girard F, Antoni M. Influence of substrate heating on the evaporation dynamics of pinned water droplets. Langmuir. 2008;24:11342-11345.

43. Girard F, Antoni M, Sefiane K. On the effect of Marangoni flow on evaporation rates of heated water drops. Langmuir. 2008;24:9207-9210.

44. Frastia L, Archer AJ, Thiele U. Dynamical model for the formation of patterned deposits at receding contact lines. Phys Rev Letts. 2011; 106:077801.

45. Sefiane K, Bennacer R. An expression for droplets evaporation incorporating thermal effects. J Fluid Mech. 2011;667:260-271.

46. Kim J-H, Ahn SI, Kim JH, Zin W-C. Evaporation of water droplets on polymer surfaces. Langmuir. 2007;23:6163-6169.

47. Orejon D, Sefiane K, Shanahan MER. Stick-slip of evaporating droplets: substrate hydrophobicity and nanoparticle concentration. Langmuir. 2011;27:12834-12843.

48. Shin DH, Lee SH, Jung J-Y, Yoo JY. Evaporating characteristics of sessile droplet on hydrophobic and hydrophilic surfaces. Microelectronic Eng. 2009;86:1350-1353.

49. Guena G, Poulard C, Cazabat AM. The leading edge of evaporating droplets. J Coll Interf Sci. 2007;312:164-171.

50. de Gennes PG. Wetting: statics and dynamics. Rev Mod Phys. 1985; 57:827-863.

51. Heslot F, Cazabat AM, Levinson P, Fraysse N. Experiments on wetting on the scale of nanometers - influence of the surface-energy. Phys Rev Lett. 1990;65:599-602.

52. Vuilleumier R, Ego V, Neltner L, Cazabat AM. Tears of wine - the stationary state. Langmuir. 1995;11:4117-4121.

53. Poulard C, Damman P. Control of spreading and drying of a polymer solution from Marangoni flows. Europhys Lett. 2007;80:64001.

54. Shahidzadeh-Bonn N, Fafai S, Azouni A, Bonn D. Evaporating droplets. J Fluid Mech. 2006;549:307-313.

55. Weon BM, Je JH. Self-pinning by colloids confined at a contact line. Phys Rev Lett. 2013;110:028303.

56. Dong A, Chen J, Oh SJ, Koh W, Xiu F, Ye X, Ko D-K, Wang KL, Kagan CR, Murray CB. Multiscale periodic assembly of striped nanocrystal super lattice films on a liquid surface. Nano Lett. 2011; 11:841-846.

57. Sangani AS, Lu C, Su K, Schwarz JA. Capillary force on particles near a drop edge resting on a substrate and a criterion for contact line pinning. Phys Rev E. 2009;80:011603.

58. Dussan VEB. On the spreading of liquids on solid surfaces: static and dynamic contact lines. Annu Rev Fluid Mech. 1979;11:371-400.

59. Lyushnin AV, Golovin AA, Pismen LM. Fingering instability of thin evaporating liquid films. Phys Rev E. 2002;65:021602.

60. Craster RV, Matar OK, Sefiane K. Pinning, retraction, and terracing of evaporating droplets containing nanoparticles. Langmuir. 2009;25: 3601-3609.

61. L Chen, Evans JRG, Arched structures created by colloidal droplets as they dry. Langmuir. 2009;25:11299-11301.

62. Nguyen TAH, Hampton MA, Nguyen AV. Evaporation of nanoparticle droplets on smooth hydrophobic surfaces: the inner coffee ring deposits. J Phys Chem C. 2013;117:4707-4716.

63. Ray MA, Kim H, Jia L. Dynamic self-assembly of polymer colloids to form linear patterns. Langmuir. 2005;21:4786-4789.

64. Morales V, Parlange J, Wu M, Zhang W, Sang W, Steenhuis T. Interaction of colloidal particles with the air-water-solid interface of a drying droplet. Langmuir. 2013;29:1831-1840.

65. Bhardwaj R, Fang X, Somasundaran P, Attinger D. Self-Assembly of colloidal particles from evaporating droplets: role of DLVO inter- actions and proposition of a phase diagram. Langmuir. 2010;26: 7833-7842.

66. Wang J, Evans JRG. Segregation in multicomponent ceramic colloids during drying of droplets. Phys Rev E. 2006;73:021501.

67. Majumder M, Rendall CS, Eukel JA, Wang JYL, Behabtu N, Pint CL, Liu T-Y, Orbaek AW, Mirri F, Nam J, Barron AR, Hauge RH, Schmidt HK, Pasquali M. Overcoming the "coffee stain" effect by compositional Marangoni- flow-assisted drop-drying. J Phys Chem B. 2012;116:6536-6542.

68. Bigioni TP, Lin X-M, Nguyen TT, Corwin EI, Witten TA, Jaeger HM, Kinetically driven self-assembly of highly ordered nanoparticle monolayers. Nature Mater. 2006;5:265-270.

69. Marin AG, Gelderblom H, Lohse D, Snoeijer JH. Order-to-disorder transition in ring-shaped colloidal stains. Phys Rev Lett. 2011;107: 085502 .

70. Yan Q, Gao L, Sharma V, Chiang Y-M, Wong CC. Particle and substrate charge effects on colloidal self-assembly in a sessile drop. Langmuir 2008;24:11518-11522.

71. Yunker PJ, Still T, Lohr MA, Yodh AG. Suppression of the coffeering effect by shape dependent capillary interactions. Nature. 2011; 476:303-311.

72. Yunker PJ, Lohr MA, Still T, Borodin A, Durian, DJ, Yodh AG. Effects of particle shape on growth dynamics at edges of evaporating drops of colloidal suspensions. Phys Rev Lett. 2013;110:035501.

73. Pauchard L, Couder Y. Invagination during the collapse of an inhomogeneous spheroidal shell. Europhys Lett. 2004;66:667-673.

74. Yarin AL, Szczech JB, Megaridis CM, Zhang J, Gamota DR. Lines of dense nanoparticle colloidal suspensions evaporating on a flat surface: formation of non-uniform dried deposits. J Coll Interf Sci. 2006;294:343-354.

75. Vakarelski IU, Chan DYC, Nonoguchi T, Shinto H, Higashitani K. Assembly of gold nanoparticles into microwire networks induced by drying liquid bridges. Phys Rev Lett. 2009;102:058303.

76. Sharma R, Lee CY, Choi JH, Chen K, Strano MS. Nanometer positioning, parallel alignment,and placement of single anisotropic nanoparticles using hydrodynamic forces in cylindrical droplets. Nano Lett. 2007;7:2693-2700.

77. Chakrapani N, Wei B, Carrillo A, Ajayan PM, Kane RS. Capillaritydriven assembly of two dimensional cellular carbon nanotube foams. PNAS. 2004;101:4009-4012.

78. Harris DJ, Hu H, Conrad JC, Lewis JA. Patterning colloidal films via evaporative lithography, Phys Rev Lett. 2007;98:148301.

79. Nguyen VX, Stebe KJ. Patterning of small particles by a surfactantenhanced Marangoni-Benard instability. Phys Rev Lett. 2002;88: 1645011-1645014.

80. Senses E, Black M, Cunningham T, Sukhishvili SA, Akcora P. Spatial ordering of colloids in a drying aqueous polymer droplet. Langmuir. 2013;29:2588-2594.

81. Tarasevich YY, Vodolazskaya IV. Desiccating colloidal sessile drop: dynamics of shape and concentration. Colloid Polym Sci. 2011;289: 1015-1023.

82. Maheshwari S, Zhang L, Zhu Y, Chang H-C. Coupling between precipitation and contact line dynamics: multiring stains and stick-slip motion. Phys Rev Lett. 2008;100:044503.

83. Xu J, Xia J, Hong SW, Lin Z, Qiu F, Yang Y. Self-assembly of gradient concentric rings via solvent evaporation from a capillary bridge. Phys Rev Lett. 2006;96:066104.

84. Lin Z, Granick S. Patterns formed by droplet evaporation from a restricted geometry. J Am Chem Soc. 2005;127:2816-2817.

85. Kajiya T, Nishitani E, Yamaue T, Doi M. Piling-to-buckling transition in the drying process of polymer solution drop on substrate having a large contact angle. Phys Rev E. 2006;73:011601.

86. Gorand Y, Pauchard L, Calligari G. Mechanical instability induced by the desiccation of sessile drops. Langmuir 2004;20:5138-5140.

87. Willmer D, Baldwin KA, Dwartnik C, Fairhurst DJ. Growth of solid conical structures during multistage drying of sessile poly(ethylene oxide) droplets. Phys Chem Chem Phys. 2010;12:3998-4004.

88. Takhistov P, Chang H-C. Complex stain morphologies. Ind Eng Chem Res. 2002;41:6256-6269.

89. Kaya D, Belyi VA, Muthukumar M. Pattern formation in dryng droplets of polyelectrolyte and salt. J Chem Phys. 2010;133:114905.

90. Larson RG, Lopez MA, Lim DW, Lahann J. Complex protein patterns in drying droplets. MRS Proceedings. 2010;1273:1273MM03-01.

91. Leiterer J, Emmerling F, Panne U, Christen W, Rademann K. Tracing coffee tabletop traces. Langmuir. 2008;24:7970-7978. 
92. Takhistov P, Chang H-C. Complex Stain Morphologies. Ind Eng Chem Res. 2002;41:6256-6269.

93. Mullins W, Sekerka RF. Morphological stability of a particle growing by diffusion or heat flow. J. Appl. Phys. 1963;34:323-329.

94. Tarasevich YY, Pravoslavnova DM. Segregation in desiccated sessile drops of biological fluids. Euro Phys J E. 2007;22:311-314.

95. Trantum JR, Wright DW, Haselton FR. Biomarker-mediated disruption of coffee-ring formation as a low resource diagnostic indicator. Langmuir 2012;28:2187-2193.
96. Wen JT, Ho C-M, Lillehoj PB. A Coffee-ring biosensor for rapid protein detection. Langmuir. 2013;29:8440-8446.

97. Brutin D, Sobac B, Loquet B, Sampol J, Pattern formation in drying drops of blood. J Fluid Mech. 2011;667:85-95.

98. Kim N, Li Z, Hurth C, Zenhausern F, Chang S-F, Attinger D. Identification of fluid and substrate chemistry based on automatic pattern recognition of stains. Anal Meth. 2012;4:50-57.

Manuscript received Sept. 1, 2013, and final revision received Dec. 24, 2013. 